\title{
(Photo-)crosslinkable Gelatin Derivatives for Biofabrication
}

\section{Applications}

Jasper Van Hoorick ${ }^{1,2+}$, Liesbeth Tytgat ${ }^{1,2+}$, Agnes Dobos $^{3,4}$, Heidi Ottevaere ${ }^{2}$, Jürgen Van Erps ${ }^{2}$, Hugo Thienpont ${ }^{2}$, Aleksandr Ovsianikov ${ }^{3,4}$, Peter Dubruel ${ }^{1}$, Sandra Van Vlierberghe ${ }^{1,2^{*}}$

1. Polymer Chemistry \& Biomaterials Group - Centre of Macromolecular Chemistry (CMaC) Department of Organic and Macromolecular Chemistry, Ghent University, Krijgslaan 281, S4-Bis, 9000 Ghent, Belgium.

2. Brussels Photonics (B-PHOT) - Department of Applied Physics and Photonics, Vrije Universiteit Brussel and Flanders Make, Pleinlaan 2, 1050 Brussels, Belgium.

3. Research Group 3D Printing and Biofabrication, Institute of Materials Science and Technology, TU Wien, Getreidemarkt 9, 1060 Vienna, Austria

4. Austrian Cluster for Tissue Regeneration, (http://www.tissue-regeneration.at)

${ }^{+}$Both authors contributed equally

${ }^{*}$ Corresponding author

\section{$\underline{\text { Abstract }}$}

Over the recent decades gelatin has proven to be very suitable as an extracellular matrix mimic for biofabrication and tissue engineering applications. However, gelatin is prone to dissolution at typical cell culture conditions and is therefore often chemically modified to introduce (photo-)crosslinkable functionalities. These modifications allow to tune the material properties of gelatin, making it suitable for a wide range of biofabrication techniques both as a bioink and as a biomaterial ink (component). The present review provides a non-exhaustive overview of the different reported gelatin modification strategies to yield crosslinkable materials that can be used to form hydrogels suitable for 
biofabrication applications. The different crosslinking chemistries are discussed and classified according to their crosslinking mechanism including chain-growth and step-growth polymerization. The step-growth polymerization mechanisms are further classified based on the specific chemistry including different (photo-)click chemistries and reversible systems. The benefits and drawbacks of each chemistry are also briefly discussed. Furthermore, focus is placed on different biofabrication strategies applying inkjet, deposition and light-based additive manufacturing techniques, and the applications of the obtained 3D constructs.

\section{Introduction}

During the last two decades, biofabrication has gained increasing attention within the field of tissue engineering and regenerative medicine. This is a consequence of the potential to fabricate complex, patient-specific constructs that closely resemble the complexity and heterogeneity of native tissues and organs in an automated way according to a computer-aided design (CAD) [1-3]. Within the field of biofabrication, two main strategies are currently explored, which are either based on the use of a (bio)material support resembling the extracellular matrix (ECM) or solely on cells along with cell secreted materials. When a biomaterial is applied, it can either be used as a biomaterial ink or a bioink depending on the composition. The term biomaterial ink refers to material processing via additive manufacturing and subsequent cell seeding, while the term bioink corresponds to the use of a mixture which already contains cells prior to processing via additive manufacturing $[1,3]$.

Gelatin as a bioink or a biomaterial ink - throughout the present manuscript, the term bio(material)ink is used when referring to both - has attracted considerable attention over the years as it is derived from collagen, which is the main constituent of the natural ECM of mammals $[4,5]$. It is a denatured protein constituting 18 different amino acids characterized by a repetitive unit of glycine $-X-Y$ in which $X$ and $Y$ can be several different amino acids $[6,7]$. However, $X$ and $Y$ predominantly consist of proline and hydroxyproline which enables the formation of triple helices or physical crosslinks via interchain hydrogen bonds $[6,7]$. As a consequence, the material is characterized by a dissociation 
temperature around $30-35^{\circ} \mathrm{C}[6,8,9]$. As a result, it dissolves at elevated temperatures, while forming a swollen hydrogel below this phase change temperature [6,9-11]. Additionally, the presence of the tripeptide arginine-glycine-aspartic acid (RGD) in the protein backbone results in cell-interactive properties [12,13]. Furthermore, it is enzymatically degradable by metalloproteases such as collagenase, which cleaves sequences such as Gly-Pro-Gln-Gly-lle-Ala-Gly-Gln between Gly and Ile allowing cells to remodel it [11,14-17]. Due to the harsh acidic or basic denaturation process, concerns regarding immunogenicity and pathogen transmittance associated with the use of collagen are circumvented $[4,18]$. In addition, it is considered safe by the Food and Drug administration (FDA) with a wide track record in the food and pharmaceutical industry $[6,7,19]$. Furthermore, gelatin is a byproduct from the meat industry making it very attractive from an economical point of view [20]. However, due to the solubility at body temperature, the material was originally only applied as a temporary cell carrier to enable more straightforward cell manipulation [20]. To overcome this limitation, strategies were developed to stabilise the material at physiological conditions via the formation of chemical crosslinks. A common approach in this respect, consists of coupling the primary amines present in (hydroxy)lysine and ornithine with the carboxylic acids from aspartic and glutamic acid using carbodiimide chemistry thereby resulting in a zero length crosslinked hydrogel network $[18,21]$. Alternatively, the nucleophilic functionalities of gelatin can be crosslinked using glutaraldehyde [22]. However, these stabilisation techniques offer limited control over the design of the obtained construct, as the material manipulation window is limited in time with little control over the crosslinking process.

A realm shift occurred in 2000 when Van den Bulcke et al. developed and patented the first photocrosslinkable gelatin derivative (i.e. gelatin-methacrylamide (gel-MA))[22,23]. Photopolymerization exhibits attractive capabilities in terms of material processing including highly controllable gelation kinetics and predictable degradation enabling convenient and straightforward material processing for biofabrication purposes $[9,23,24]$. The functionalization occurs by reacting the primary amines in the side chains of (hydroxy)lysine and ornithine with methacrylic anhydride, resulting in the formation of 
methacrylamide moieties [9]. Ever since, gel-MA has been applied for a plethora of biofabrication and tissue engineering strategies either as a standalone material or co-crosslinked with other (synthetic) materials (e.g. PEG) to form biohybrid hydrogels. As a result, it became one of the gold standards in the field $[2,8,25-33]$. Following this success, it has even started to bridge the gap between academia and industry as it is offered commercially by several companies as a bio(material)ink for research purposes [34,35]. Besides gel-MA, several other (photo-)crosslinkable gelatin derivatives have emerged (Figure 1: Non-exhaustive overview of different crosslinkable gelatins including their method of preparation classified according to their crosslinking chemistry: Chain-growth derivatives (blue): gel-MOD/gel-MA(A) [9], gel-MOD-AEMA (B) [16], gel-MA-DA (C) [36], GMA (D) [37], gel-AA (E) [38], gelatin-acrylamide (F) [39], gel-BTHE (G) [40], gel-Boc-AEMA (H) [41], methacrylated poly(ethylene glycol)-modified gelatin(MPG) (I) [42,43], gelatin-PEG (K) [44]; Thiolated gelatins suitable for disulphide chemistry of thiol-ene chemistry (purple): gel-SH (J) [44,45], gel-SH (L) [45,46], aminated gelatin (M) [5,47], aminated-thiolated-gelatin (N) [47], gelatin-Cys-2-MPD (O) [48], gelatin-Cys (P) [48], gel-PEG-Cys (Q) [49], gelatin-TBA-MNA (R) [50], gel-S (S) [51,52], gelatin-thiobutyrolacton (T) [53]; Derivatives for enzymatic crosslinking (white): gelatin-tyramine (U) [4,11], gelatin/tyramine/heparin (V) [4]; Derivatives suitable for photo-oxidation (green): gelatin-FA (W) [54], gelatin-FI (X) [55] gelFGE (Y) [56,57]; $\pi-\pi$ cycloaddition (yellow): gel-MFVF (Z) [58], gel-AC ( $\alpha$ ) [59], gel-NC ( $\beta$ ) [59]; Derivatives suitable for Diels-Alder click (light green): gel-furan $(\gamma)$ [60], gel-FGE $(\delta)$ [61], gel-NB $(\varepsilon)$

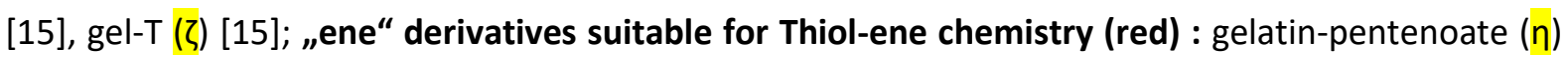
[53], gel-AGE ( $\theta)$ [62], gel-VE ( $)$ [63], gel-NB (к) $[46,64]$, gel-NB $(\lambda)[17,65]$, gel-NB $(\mu)$ [66] (image continued on the next page)

). These derivatives can be subdivided into different classes based on the applied crosslinking mechanism including chain-growth (Figure 1 blue) and step-growth polymerization. Within the stepgrowth classification, several other subclasses can be distinguished based on the applied crosslinking chemistry: thiol-ene (photo-)click chemistry: (thiols: Figure 1 purple; enes: figure 1 red), disulphide linkages (Figure $1 \& 3$ purple), Diels-Alder click (Figure $1 \&$ 3: light green), Schiffs-base formation 
(Figure 3 grey), $\pi-\pi$ cycloaddition (Figure $1 \& 3$ : yellow), photooxidation (Figure $1 \&$ 3: green) and enzymatic based crosslinking (Figure $1 \& 3$ : white).

Therefore, the present review aims to provide a helicopter view on all aspects related to the use of gelatin for biofabrication applications starting with raw materials and ending with final applications. Throughout the review, attention is paid to the physical and chemical properties, different chemical modification strategies and their implications on material and processing properties. Furthermore, an overview of different applied additive manufacturing technologies is provided including some final biomedical applications. Finally, a non-exhaustive overview of all presented gelatin derivatives and their processability potential towards specific additive manufacturing technologies is provided. 


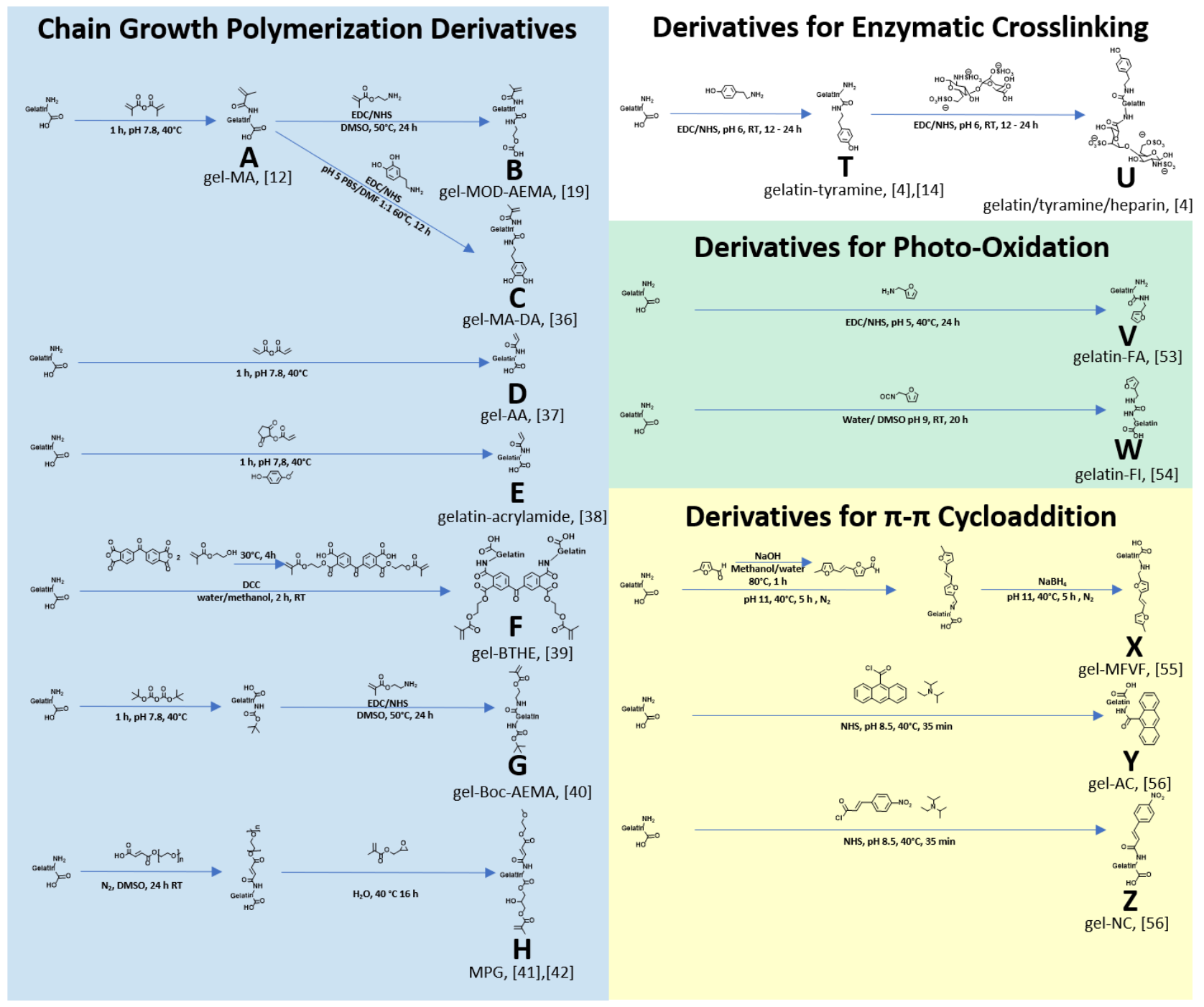

Figure 1: Non-exhaustive overview of different crosslinkable gelatins including their method of preparation classified according to their crosslinking chemistry: Chain-growth derivatives (blue): gelMOD/gel-MA(A) [9], gel-MOD-AEMA (B) [16], gel-MA-DA (C) [36], GMA (D) [37], gel-AA (E) [38], gelatin-acrylamide (F) [39], gel-BTHE (G) [40], gel-Boc-AEMA (H) [41], methacrylated poly(ethylene glycol)-modified gelatin(MPG) (I) $[42,43]$, gelatin-PEG (K) [44]; Thiolated gelatins suitable for disulphide chemistry of thiol-ene chemistry (purple): gel-SH (J) [44,45], gel-SH (L) [45,46], aminated gelatin (M) [5,47], aminated-thiolated-gelatin (N) [47], gelatin-Cys-2-MPD (O) [48], gelatin-Cys (P) [48], gel-PEG-Cys (Q) [49], gelatin-TBA-MNA (R) [50], gel-S (S) [51,52], gelatin-thiobutyrolacton (T) [53]; Derivatives for enzymatic crosslinking (white): gelatin-tyramine (U) [4,11], gelatin/tyramine/heparin (V) [4]; Derivatives suitable for photo-oxidation (green): gelatin-FA (W) [54], gelatin-FI (X) [55] gelFGE (Y) [56,57]; $\pi-\pi$ cycloaddition (yellow): gel-MFVF (Z) [58], gel-AC ( $\alpha$ ) [59], gel-NC ( $\beta$ ) [59]; Derivatives suitable for Diels-Alder click (light green): gel-furan $(\gamma)$ [60], gel-FGE $(\delta)$ [61], gel-NB $(\varepsilon)$

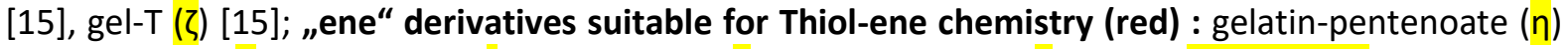
[53], gel-AGE ( $\theta)$ [62], gel-VE ( $)$ [63], gel-NB (k) $[46,64]$, gel-NB $(\lambda)[17,65]$, gel-NB $(\mu)[66]$ (image continued on the next page) 


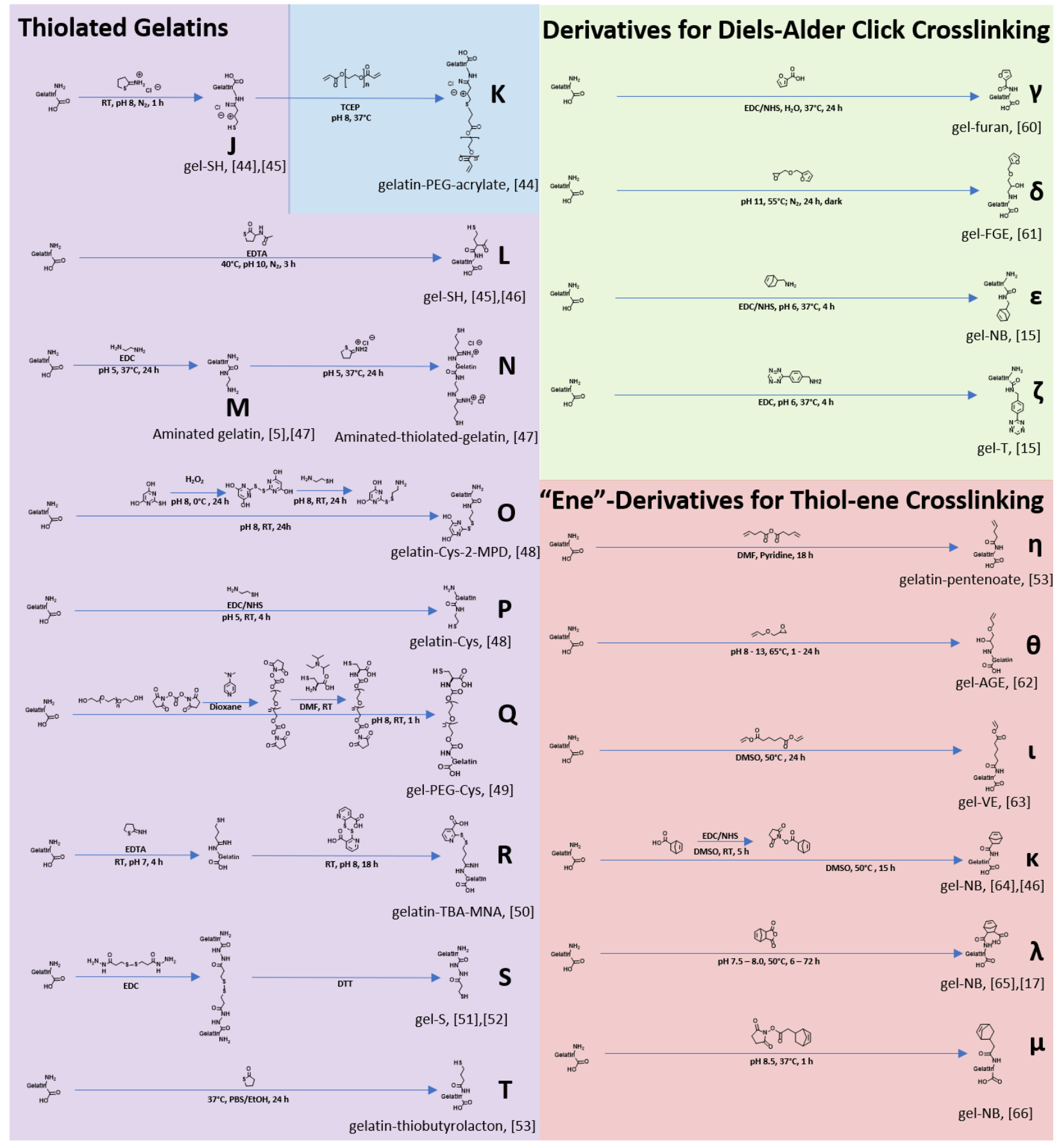




\section{Classification according to crosslinking mechanism}

\subsection{Crosslinking via Chain-Growth Polymerization}

The most commonly used crosslinkable gelatin derivatives take advantage of a chain growth polymerization crosslinking approach. Here, crosslinking occurs by polymerizing reactive functionalities (typically (meth)acrylates/(meth)acrylamides) immobilized onto gelatin resulting in the formation of short oligomer/polymer kinetic chains in between the gelatin chains $[9,16,26,38,62,64]$

(Figures 2. A \& C). Consequently, a polymer network is generated containing both gelatin polypeptide chains and synthetic oligomer/polymer chains. Crosslinking usually occurs via photo-polymerization. However, also other initiating systems can be applied (i.e. APS/TEMED) $[21,31,67]$. The benefits of chain-growth polymerization systems include straightforward material handling, consisting of material dissolution and addition of a suitable (photo-)initiator prior to crosslinking without the need for any additional crosslinker. Furthermore, the introduction of methacrylamides to gelatin (gel-MA) involves a straightforward single step reaction resulting in a plethora of applications. (Figure 1: A) $[8,9,25,28,33,68-75]$. Besides this success, other derivatives have also been reported to further tune/improve the material properties. Examples include the introduction of more reactive functionalities (i.e. acrylates/acrylamides (Figure $1 \mathrm{E}, \mathrm{F}$ ) or gelatin-PEG-acrylate (Figure $1 \mathrm{~K}$ ) $[38,44]$ ) to improve the crosslinking rate. Other attempts aim to increase the mechanical properties of crosslinked gelatin by introducing more crosslinkable sites through modification of the carboxylic acids of glutamic- and aspartic acid, being predominantly present in gelatin in comparison to lysine and hydroxylysine which are usually functionalized. Using this strategy, (additional) methacrylates could be introduced via carbodiimide coupling of 2-aminoethyl methacrylate yielding gel-MOD-AEMA (Figure $1 \mathrm{~B}$ ) and gel-Boc-AEMA (Figure $1 \mathrm{H}$ ) $[16,41,76]$. Finally, Ding et al. explored the incorporation of photocrosslinkable functionalities which already include a photoinitiating moiety (i.e. a benzophenone group linked to the methacrylate functionalities), thereby overcoming the need for the addition of a potentially cytotoxic photoinitiator (PI) (Figure $1 \mathrm{G}$ ) [40,77]. 
In comparison to the second predominant gelatin crosslinking chemistry (i.e. thiol-ene based systems (vide infra)) in which thiolated crosslinkers are applied to crosslink an "ene" functionalized material, chain-growth gelatin solutions remain stable for longer time periods above the dissociation temperature (cfr. the half-life of dithiotreitol (DTT), a commonly applied crosslinker, shifts from $11 \mathrm{~h}$ at $0^{\circ} \mathrm{C}$ to only $0.2 \mathrm{~h}$ at $40^{\circ} \mathrm{C}$ at $\mathrm{pH} 8.5$ whereas during modification gel-MA can be kept at $40^{\circ} \mathrm{C}$ for at least 24 hours without any problems). This thermal stability is typically required during most additive manufacturing processes or for cell encapsulation experiments $[16,78]$. Moreover, chain-growth systems typically yield stiffer hydrogels in comparison to step-growth hydrogels as a result of the kinetic polymer chains which can be a benefit towards stiffer tissue engineering applications including intervertebral discs (i.e. Storage modulus (G') ranging from 8 - $93 \mathrm{kPa}$ [79]) (Figure $5 \mathrm{~A}$ ) [64].

Drawbacks associated with chain growth hydrogels include the formation of a more heterogeneous network due to the presence of these kinetic chains rendering the material prone to shrinkage during crosslinking [40]. Furthermore, the kinetic profile of free radical chain-growth polymerizations is usually more complicated as a consequence of chain-length issues and reaction diffusion limitations resulting in termination which leads to a diminished control over the number of reacted functionalities $[80,81]$. Moreover, the crosslinking reaction is prone to oxygen inhibition due to rapid radical scavenging by oxygen molecules resulting in the formation of hydroxyperoxides and alcohols, which is undesirable upon targeting cell encapsulation and also influences reaction reproducibility [82]. These oxygen inhibition effects can be circumvented by using higher PI concentrations in combination with higher spatiotemporal energy (i.e. higher UV intensity, longer irradiation times) to crosslink the material [82]. As a result, chain-growth crosslinking typically requires more energy and more $\mathrm{PI}$ compared to thiol-ene-based, step-growth hydrogels (vide infra) [64]. However, both higher PI concentrations and higher light intensities can induce cellular damage rendering them less favourable for direct cell encapsulation $[62,82]$. As a consequence, increasing attention is paid towards the development of alternative crosslinking chemistries. 


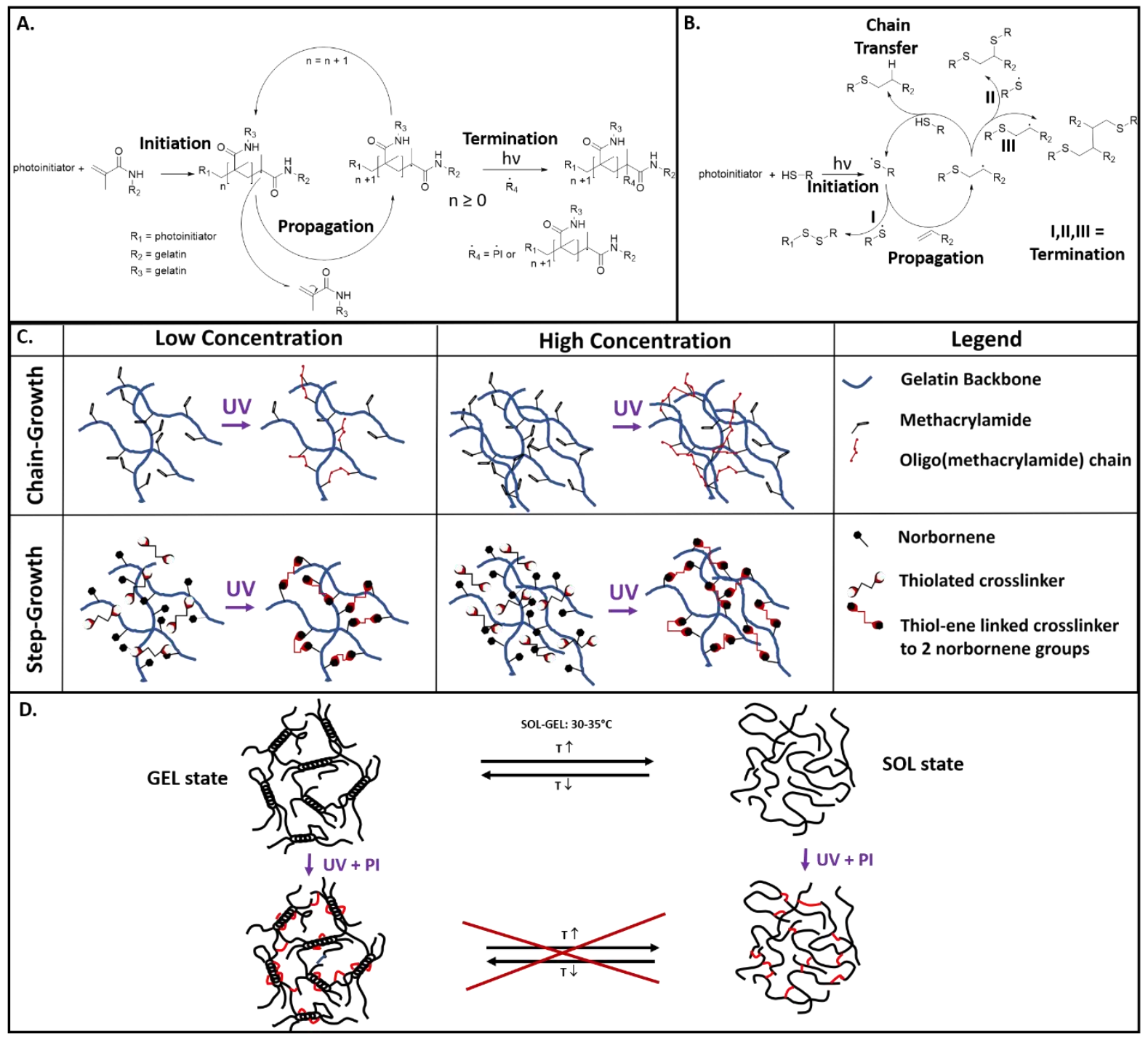

Figure 2: Illustration of the chain-growth (A.) vs step-growth (B.) crosslinking mechanism using thiolene photoclick chemistry. Influence of applied chemistry on network properties (C.) demonstrating the presence of kinetic chains in chain-growth crosslinking approaches as compared to a thiol-ene photoclick-based system. (Adapted from [64]) Influence of physical gelation on network density and associated mechanical properties (D.). (Adapted with permission from [16] copyright 2017 ACS (https://pubs.acs.org/doi/abs/10.1021\%2Facs.biomac.7b00905))

\subsection{Crosslinking via Step-Growth Polymerization}

The second major class of photo-crosslinkable gelatin hydrogels involves a step-growth polymerization crosslinking approach. A step-growth mechanism typically occurs between two complementary reactive groups which can ideally only react with one another [81]. Of specific interest in this area is the use of "click chemistry", a concept introduced by Sharpless et al. in 2001. Click chemistry involves chemical reactions which typically occur very fast (i.e. "spring-loaded"), with a high degree of control 
at high yields under relatively mild conditions (i.e. physiologically stable), without the formation of toxic side products, making them ideal to crosslink hydrogels for biomedical applications $[60,83]$. A non-exhaustive overview of step-growth crosslinking chemistries applied for gelatin hydrogels is presented in Figure 3. Furthermore, an overview of the modifications on processability using additive manufacturing technologies is presented in section 7 , table 1. 


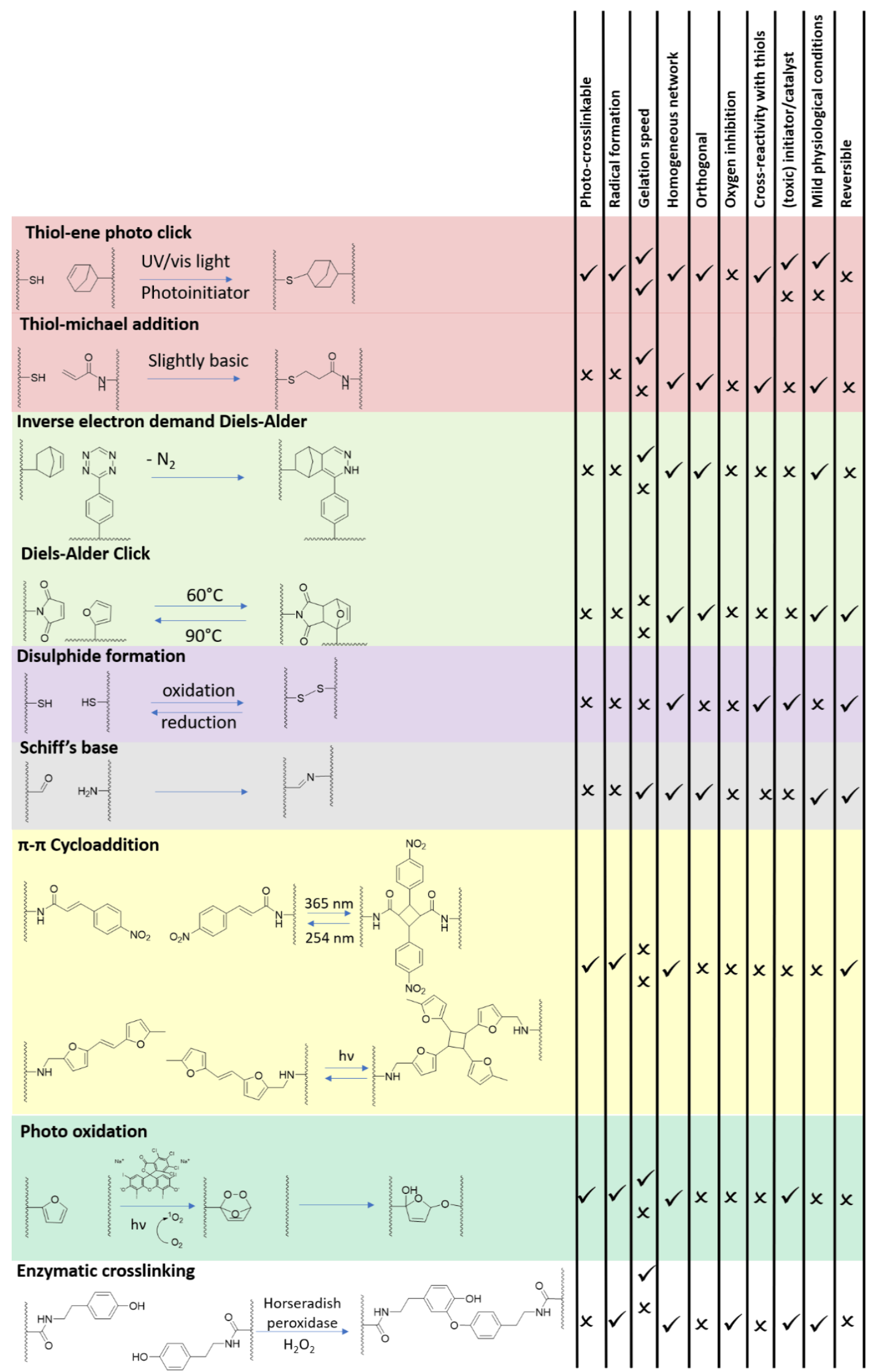

Figure 3: Overview of different step-growth crosslinking chemistries applied to gelatin including: thiolene (photo-)click (red), Diels-Alder click (light green), disulphide formation (purple), Schiff's base formation (grey), cycloaddition (yellow), photo-oxidation (dark green) and enzymatic crosslinking (white). 


\subsubsection{Thiol-ene Photo Click Crosslinking}

The most common "click" crosslinking chemistry applied for gelatin hydrogels is thiol-ene ("photo"-) click chemistry (Figure 2. B and Figure 3). These systems can be applied to form networks by reacting any thiol with any 'ene' functionality either following a light-induced, radical-mediated thiol-ene reaction or by the formation of an anionic species resulting in a thiol Michael-type addition (vide infra) [81]. The light-induced reaction usually proceeds via hydrogen abstraction of the thiol resulting in the formation of a thiyl radical which can be generated either in the presence or absence of a photoinitiator (Figure 2 B: initiation). Next, an anti-Markovnikov addition of this radical to the double bond present in the 'ene' species occurs (Figure 2 B: propagation) $[81,84]$. After the addition, a radical chain transfer occurs between the formed carbon-centered radical to another thiol group thereby forming another thyil radical (Figure 2 B: chain transfer) [84]. Finally, termination occurs when two radical species recombine forming either a disulphide bridge (i.e. when two thyil radicals combine (Figure 2 B: Termination I)), a carbon-sulfur bond (I.e. coupling of a thiyl radical with a carbon-centered radical (Figure 2 B: Termination II)) or a carbon-carbon bond (i.e. coupling of two carbon centered radicals (Figure 2 B: Termination III)) [84]. The rate limiting step in this process is the chain transfer step, therefore, thiol-ene reactions proceed slower in systems where this chain transfer is hindered (e.g. in the presence of methyl in methacrylates leading to steric hindrance in contrast to acrylates) [84]. Furthermore, due to the electrophilic nature of the thyil radical, electron rich enes typically undergo the fastest reaction $[84,85]$. However, norbornene, methacrylate, styrene and conjugated diene functionalities are exceptions to this rule. Thiol-norbornene reactions are extremely fast due to the ring-strain relief upon thyil addition and the rapid hydrogen abstraction rate $[62,65,85]$. The methacrylates, styrenes and conjugated dienes, are all characterised by a conjugated system, enabling radical stabilisation due to mesomeric delocalisation over multiple atoms, which results in slow hydrogen abstraction rates and concomitant lower reactivity [85]. Consequently, the reaction rate of different functionalities relative to each other exhibits the following trend [85]: 
Norbornene $>$ vinyl ether $>$ propenyl $>$ alkene $\sim$ vinyl ester $>\mathrm{N}$-vinyl amides $>$ allyl ether $>\mathrm{N}$-vinyl amides $>$ acrylate $>$ acrylonitrile $\sim$ methacrylate $>$ styrene $>$ conjugated dienes.

In general, the reaction can proceed with any type of non-sterically hindered 'ene' functionality. However, if a true step-growth polymerization reaction is pursued, an 'ene' functionality, which cannot undergo competitive chain-growth homo-polymerization (i.e. norbornenes and vinyl ethers) is preferred [81]. As a consequence, superior control over the reaction and concomitant homogeneity within the resulting network is obtained [81]. To develop a thiol-ene photo-crosslinkable gelatin, it has to contain 'ene' functionalities (typically norbornene, vinyl esters, pentenoyls, allyl ethers or acrylates) which can be crosslinked using a multi-functional, thiolated crosslinker (e.g. DTT) $[46,53,62,64,65,86,87]$ (Figure 1 (red), Figure 2. C and Figure 3 (red)). Alternatively, gelatin is functionalized with thiols and crosslinked using a multi-functional 'ene' crosslinker (e.g. polyethylene glycol-diacrylate PEGDA, gel-NB or gel-AA) [46,53,86,88,89] (Figure 1 (purple)).

Thiol-ene 'photo-click' hydrogels pose a benefit over chain-growth hydrogels (vide supra) as more homogeneous networks are formed with a higher conversion of the functional groups, in combination with less shrinkage during crosslinking resulting in less post-polymerization stress due to the highly orthogonal nature of the reaction $[62,90,91]$. Additionally, the crosslinking reaction is not susceptible to oxygen inhibition and exhibits lower radical concentrations (i.e. at least one order of magnitude below chain growth systems) and faster reaction rates as reflected by shorter gel-point times, making them more suitable for cell encapsulation (Figure 5 B) $[62,64,65,81,84,90,92,93]$. Furthermore, the number of reacted functionalities can be fully controlled by varying the thiol-ene ratio prior to crosslinking $[64,81,90]$. As a consequence, when using ene functionalities which cannot undergo competitive homo polymerization (i.e. vinyl ether, norbornene) pendant ene or thiol functionalities can be retained in the material after crosslinking, thereby allowing post crosslinking grafting or photomicropatterning with other compounds (i.e. integrin binding sites, growth factors, proteins,... ) with spatiotemporal control (e.g. when using photomasks, lithography approaches) thereby providing a better biomimetic matrix $[14,94,95]$. Drawbacks include the necessity of a multifunctional, thiolated 
crosslinker present in the reaction mixture which can be susceptible to cross-reactivity with other thiols leading to disulphide formation, especially in oxophilic aqueous systems, thereby resulting in poor stability of the crosslinkable solution[96-99]. The probability for disulphide formation increases over time at elevated temperatures which is essential to maintain gelatin solubility during the main part of additive manufacturing technologies (with the exception of two-photon polymerization (2PP) [78]. Additionally, these thiolated crosslinkers can exhibit reactivity with thiol functionalities present in living cells resulting in cellular damage [100]. Furthermore, the obtained hydrogels are generally characterised by lower storage moduli as compared to their chain-growth counterparts (i.e. $0.6-46$ kPa for thiol-ene [62] vs 0.07 [44] - $368 \mathrm{kPa}$ [101] for chain growth, Figure 5 A) [64,65]. Typical gelatin derivatives prone to thiol-ene photoclick crosslinking reactions are depicted in red (enes) and purple (thiolated gelatins) in Figure 1. Examples of gelatin derivatives with ene functionalities include: gelatinpentenoate $(\eta)$ [53], gel-AGE $(\theta)[62]$, gel-VE $(\iota)[63]$, gel-NB $(\kappa, \lambda \& \mu)[17,64,65]$. Examples of thiolated gelatin derivatives include: gel-SH (J) [44,45], gel-SH (L) [45,46], aminated-thiolated-gelatin (M) [47], gelatin-Cys (P) [48], gel-PEG-Cys (Q) [49], gelatin-thiobutyrolacton (T) [53];

\subsubsection{Thiol-Michael Addition Based Crosslinking}

Thiol-Michael addition is the reaction between a thiol and an electron deficient, activated double bond (i.e. alpha, beta unsaturated double bonds: acrylates, acrylamides; maleimides; vinyl sulphones fumarate esters; acrylonitrile; cinnamates and crotonates) via a slightly alkaline or nucleophilic catalysed mechanism [102,103] (Figure 3 (red)). Thiol-Michael type additions are highly specific nucleophilic additions, which take place without the formation of potentially harmful side products (i.e. radical species) and potentially cytotoxic PI's and (UV) irradiation [52]. Additionally, in contrast to the UV induced thiol-ene reaction, no radical-radical termination side products are formed resulting in quantitative conversion [102]. Furthermore, since the reaction only requires slightly alkaline conditions, the reaction can occur at physiological $\mathrm{pH}$ [52]. Although they are typically slower compared to thiol-ene photoclick reactions, it still exhibits relatively fast crosslinking kinetics with 
reported gel-points in the range of a few minutes [89] (Figure $5 \mathrm{~B}$ ). As a consequence, thiol-Michael addition is an ideal candidate for cell encapsulation purposes $[52,89]$.

Drawbacks include the relatively fast reaction profile, without any spatiotemporal control, making it less straightforward for biofabrication applications. However, it is anticipated that when printing one component in a container containing the other component, syringe-based printing becomes accessible. Examples of gelatin derivatives which have been applied in thiol-Michael type additions include: gel-SH (Figure $1 \mathrm{~J})[44,89]$ and gel-S (Figure $1 \mathrm{~S})[51,52]$.

\subsubsection{Diels-Alder Based Click Systems}

\subsubsection{Inverse Electron Demand Diels-Alder Based Systems}

Although thiol-ene photo-click systems pose some benefits over the conventional chain-growth crosslinking systems, both mechanisms still involve harmful radical species, while for thiol-ene systems (both thiol-ene photo click as thiol-michael addition), a cross reactivity can occur with thiols present in other proteins or the cells during cell (photo-)encapsulation [100]. Therefore, researchers are also exploring alternative "click" crosslinking mechanisms [61,83,103]. Koshy et al. evaluated a norbornene-tetrazine click system which allows crosslinking in the absence of any other trigger (i.e. UV irradiation, $\mathrm{PI}$, catalyst, ...) (Figure $1 \varepsilon, \zeta$; Figure 3 (light green)) [100]. The crosslinking occurs via an inverse electron demand Diels-Alder click reaction with the formation of nitrogen (Figure 3) [94]. Furthermore, the reaction is quantitative with high atom efficiency producing only nitrogen gas as side product $[94,97]$. Due to these aspects, tetrazine chemistry is increasingly applied in the field of polymer chemistry. By preparing a gelatin-norbornene component (gel-NB, figure $1(\varepsilon)$ ) and a gelatintetrazine (gel-T, Figure $1(\zeta)$ ) a stable, non-toxic hydrogel can be obtained after mixing (ClickGel) $[94,100]$. Furthermore, the gelation time can be tuned by varying the introduced dienophile. In this respect, norbornene provides a good compromise between reaction rate and sample manipulation time after mixing [94]. Furthermore, upon encapsulation with 3T3 fibroblasts, higher cell-viabilities were observed when compared to the gel-MA reference due to the absence of harmful UV irradiation [100]. 


\subsubsection{Reversible Diels-Alder Based Click Systems}

Alternatively, Garcia-Astrain et al. explored the use of a Diels-Alder based "click" type reaction consisting of a $(4+2)$ thermo-reversible cycloaddition between a dienophile and a diene to crosslink gelatin $[61,104]$ (Figure 3 (light green)). Similar to the earlier discussed norbornene-tetrazine scheme, the reaction occurs in the absence of light, catalysts or initiators. They introduced furan functionalities as diene to gelatin (gel-FGE figure $1 \delta$ ) by reaction of the primary amines with furfuryl glycidyl ether [61]. Crosslinking of the material occurred using a modified Jeffamine-based bismaleimide as dienophile $[61,104]$. Thermal crosslinking occurred at $65^{\circ} \mathrm{C}$ during 5 hours using a $60 \mathrm{wt} \%$ gel-FGE aqueous solution with different crosslinker amounts. De-crosslinking occurred by heating the hydrogel to $90^{\circ} \mathrm{C}$ to induce the retro Diels-Alder reaction. Although the presence of water typically favours the Diels-Alder reaction over the retro Diels-Alder reaction which complicates network cleavage, the authors managed to monitor the reaction using UV-VIS spectroscopy, thereby proving reformation of the diene and dienophile [61]. Another similar Diels-Alder based crosslinking approach applied for gelatin crosslinking, involves the reaction between a furan moiety introduced onto gelatin (gel-furan, Figure $1 \gamma)$, which is crosslinked using a bismaleimide [60] (Figure 3).

Gelatin derivatives benefitting from thermoreversible Diels-Alder click chemistry include: gel-furan (Figure $1(\gamma)$ [60], and gel-FGE (Figure $1(\delta))$ [61].

\subsubsection{Reversible disulphide linkage-based crosslinking}

Another reversible system, inspired by nature, involves the application of reversible thiol/disulphide formation. Disulphide bridges between thiols can be formed using an oxidative trigger (e.g. hydrogen peroxide), while cleaving of these bonds can be realised in the presence of reducing agents (e.g. DTT or glutathione) $[98,99,105-107]$ (Figure 3 (purple)). An additional benefit associated with thiolated systems is the fact that thiolation of polymers leads up to a 140 -fold improvement of mucoadhesion via the formation of disulphide linkages between the polymer and glycoproteins within the mucosal layer $[47,48,50,99,106,108]$. Due to the combination of this property and the controllable reversible nature of this chemistry, thiolated gelatins and thiolated hydrogels in general prove to be ideal 
candidates for controlled drug release studies $[107,108]$. However, although gelatin is a protein consisting of around 18 different amino acids, it does not contain cysteine in its backbone. Therefore, disulphide chemistry can only occur after chemical introduction of thiols $[44,45,47-53]$. To this end, Van Vlierberghe et al. introduced thiols either via reaction of the primary amines with $n$ acetylhomocysteine thiolactone (Figure $1 \mathrm{~L}$ ) or Traut's reagent (Figure $1 \mathrm{~J}$ ) to yield gel-SH [45]. They reported a linear correlation between storage modulus $\mathrm{G}^{\prime}$ of the resulting gel and degree of amine substitution [53]. Using this strategy, a maximum of $300 \mu \mathrm{mol}$ thiols/g of gelatin was achieved [45]. In an attempt to further increase the number of incorporated thiols, Duggan et al. first modified a part of the carboxylic acid side chains present in the glutamic and aspartic acid amino acids by coupling ethylene diamine using carbodiimide click chemistry [47,109]. Next, both the native and the introduced amines were reacted with Traut's reagent resulting in up to $660 \mu \mathrm{mol}$ of thiols/g of gelatin. Although crosslinking of the material proved successful, no attempts were made to de-crosslink the material afterwards by reducing the disulphide linkages [47].

An important drawback associated with thiols and thiolated polymers is their short shelf life due to the potential of auto-oxidation resulting in premature crosslinking [98]. To overcome this limitation, it can be useful to incorporate protected thiols onto gelatin $[48,50,98]$. To this end, Rohrer et al. applied thiols functionalised with a 2-mercaptopyrimidine-4,6 diol as a protective leaving group for mucoadhesive drug delivery applications (gelatin-Cys-2-MPD; Figure 10 ) [48] or protected thiolated gelatin with mercaptonicotinic acid (gelatin-TBA-MNA; Figure 1 R) [50].

Other thiolation strategies include the reaction of the primary amines with gamma-thiobutyrolactone [53] (Figure $1 \mathrm{~T}$ ), reaction of the carboxylic acids with cysteamine to yield gelatin-Cys (Figure $1 \mathrm{P}$ ) [48], reaction of the carboxylic acids with 3,3'-dithiobis(propionic hydrazide) (DTP) using carbodiimide chemistry followed by cleaving of the disulphides with DTT yielding gel-S (Figure 1 S) [51,52], the reaction of the primary amines with one side of a $\mathrm{N}$-hydroxysuccinimide (NHS)-bifunctional PEG followed by linking the primary amine of cysteine to the other NHS functionality to yield gel-PEG-Cys (Figure 1 Q) [49]. 
Although the concept of a reversible hydrogel is very interesting due to the aforementioned reasons, the use of disulphide linkages as crosslinks requires the use of oxidizing chemicals including hydrogen peroxide which can induce cellular damage. Furthermore, the introduced thiols can undergo side reactions with thiolated functionalities present in the cell [100]. As a consequence, the system is less suitable for cell-encapsulation purposes $[49,110]$.

\subsubsection{Schiff's-base reaction}

Another step-growth based strategy consists of reacting the primary amine groups from gelatin with aldehyde groups from a crosslinker in the absence of light (Figure 3 grey). This chemistry is referred to as a Schiff-base crosslinking reaction $[5,111,112]$. A potential crosslinker in this respect is dextran or alginate which can be oxidized with periodate to generate aldehyde functionalities $[5,111,113]$. As a consequence, a suitable ECM mimic is formed since the natural ECM also consists of proteins and polysaccharides. Furthermore, the Schiff's base is prone to hydrolysis resulting in reversible degradation of the crosslinks afterwards [114]. In order to render gelatin water soluble at room temperature (vide infra) while introducing additional amines to increase the crosslink density, Pan et al. reacted the carboxylic acids in gelatin with ethylene diamine using carbodiimide coupling chemistry [5]. In an attempt to increase the mechanical properties of a dextran-gelatin based system formed via Schiff's base reaction, Liu et al. first introduced methacrylate functionalities to dextran prior to the periodate oxidation. As a consequence, a denser network with higher storage moduli could be obtained which was successfully applied to encapsulate vascular endothelial cells [113].

\subsubsection{Photo-Reversible Systems: $\pi-\pi$ Cycloaddition}

A proposed photo-reversible crosslinking system takes advantage of functionalities which can undergo a photo-reversible dimerization reaction by irradiation at different wavelengths [59] (Figure 3 (yellow)). Examples of such functionalities are Furanic chromophores (gel-MFVF [58] Figure $1 \mathrm{Z}$ ) and nitrocinnamate (gel-NC [59] Figure $1 \beta$ ) which can undergo a photo-reversible $(\pi 2+\pi 2)$ photocycloaddition reaction with the formation of a cyclobutane ring as a consequence (Figure 3). 
Alternatively, anthracene (gel-AC Figure $1 \alpha$ ) can also undergo a photo-reversible $(\pi 4+\pi 4)$ cycloaddition $[59,115]$.

Garcia-Astrain et al. introduced furan containing chromophores (i.e. 5-(2-(5-methyl furylene vinylene)) furancarboxyaldehyde (MFVF)) onto gelatin (gel-MFVF Figure $1 \mathrm{Z}$ ), resulting in moderate crosslinking at 365nm (Figure 3). However, photo-reversibility was not explored in the reported work [58]. Furthermore, Gattas-Asfura et al. introduced nitrocinnamate functionalities onto gelatin (gel-NC) which was successfully crosslinked in the absence of a photo-initiatior by irradiation at $365 \mathrm{~nm}$, while subsequent irradiation at $254 \mathrm{~nm}$ allowed the (partial) photocleavage of the material (Figure 3). In another attempt to develop a photo-reversible gelatin, Gattas Asfura et al. synthesized gelatin with anthracene side groups. Although the modification proved successful, quantification was not possible due to solubility issues of the synthesized gelatin [59].

The use of a photo-reversible system can have benefits in drug release applications, since the release of an active compound can be triggered locally using irradiation $[59,115,116]$. Alternatively, when using selective photocleavage with a high degree of spatiotemporal control, localised cleaving of a matrix material can aid towards guiding cell migration $[117,118]$.

In general, these systems can undergo dimerization in a relatively straightforward way when immobilized on a polymer resulting in a crosslinked network. However, there are some problems associated with the reversible reaction as typically the materials do not fully cleave upon irradiation [59]. Furthermore, using UV light and especially, UV-C light as a trigger also poses some drawbacks, since it has poor penetration depth into tissue and can additionally pose carcinogenic effects [119]. Therefore, these systems require further development before becoming viable for real biofabrication applications.

\subsubsection{Photo-Oxidation Based Systems}

In an alternative approach to previously mentioned systems, Son et al. applied irradiation to activate a functionality rendering it prone to crosslinking (Figure 3 (dark green). To this end, they took advantage of photo-oxidation of furfuryl groups introduced onto gelatin (gel-Fl; Figure $1 \mathrm{X}$ ) for 
crosslinking purposes [55]. To yield gelatin with furfuryl side groups, two modification strategies have been reported to date. The first consisted of reaction of the primary amines in gelatin with furfuryl isocyanate (FI) to yield gelatin-FI with a DS of $98 \%$ (Figure $1 \mathrm{X}$ ) [55]. In order to further optimise the reactivity, another approach applies carbodiimide crosslinking to link the primary amines of furfuryl amine (FA) to the carboxylic acids of gelatin thereby yielding gelatin-FA [54] (Figure $1 \mathrm{~W}$ ). Finally, in a third approach, the primary amines of gelatin were linked to furfuryl glycidyl ether yielding gel-FGE (Figure 1 Y) [56,57].

In order to crosslink the gelatin, first, the furfuryl groups are oxidized using the photo-induced formation of singlet oxygen by rose bengal upon irradiation with visible light. As a result, a $(2+4)$ cycloaddition of singlet oxygen to the diene of the furan moiety leads to the formation of an endoperoxide [120]. Next, the formed endoperoxides on the furfuryl rings can undergo polycondensation to form a conjugated polymer [55]. Consequently, a crosslinked network can be formed using visible light. Therefore, the need for potential damage due to UV irradiation is circumvented [55]. Additionally, the reaction is not prone to oxygen inhibition and even requires the presence of oxygen in order to proceed [121]. Furthermore, both the rose bengal (a common food dye) and the gelatin-FI as such did not exhibit any cytotoxicity [55]. However, the formation of singlet oxygen is known to negatively influence cellular survival, and is often applied in photodynamic therapy to efficiently eliminate cancerous cells $[29,93]$. As a consequence, the chemistry is less suitable for cell encapsulation. However, Mazaki et al. applied a gelatin furfuryl system (gel-FA; figure $1 \mathrm{~W}$ ) in combination with rose bengal as photosensitizer. In their study they indicated that the presence of the gel-FA (Figure $1 \mathrm{~W}$ ) exhibited a cytoprotective effect on encapsulated bone marrow-derived stromal cells in the presence of $0.05 \%$ rose bengal in comparison to a blank solution containing only rose bengal. They reported a viability of $87 \% 24$ hours after encapsulation whereas the reference solution only exhibited around $10 \%$ survival. This effect is probably a consequence of the very fast reaction of the furfuryl groups in gelatin with the generated singlet oxygen, thereby acting as a singlet oxygen scavenger [54]. 
Furthermore, Son et al. applied gel-FI (Figure $1 \mathrm{X}$ ) in combination with rose Bengal as a photosensitizer in dental pulp regenerating experiments followed by in situ irradiation, thereby outperforming the calciumhydroxide control [55].

\subsubsection{Enzymatic crosslinking}

Enzymatic crosslinking approaches can be of interest due to the mild reaction process in combination with a high specificity of the enzymes to selectively crosslink the required functionalities, rendering it very suitable for cell encapsulation purposes [4].

Additionally, it is typically faster (i.e. seconds to minutes) in comparison to most other non-light (nonclick chemistry based) induced systems (i.e. glutaraldehyde, carbodiimide) which typically require multiple hours $[4,122]$.

A typical enzymatic crosslinking approach is the use of peroxidase catalysts for the oxidation of electron donors using $\mathrm{H}_{2} \mathrm{O}_{2}$, enabling linking of polyphenols at the aromatic ring (Figure 3). To benefit from this approach, Li et al. introduced tyramine side chains onto gelatin in order to provide enzymatic crosslinking points after material injection (gelatin-tyramine; Figure $1 \mathrm{U})[4,11,122]$. Crosslinking can be achieved by the addition of horseradish peroxidase (HRP) and hydrogen peroxide (Figure 3 (white)) [4,11]. The rate of crosslinking can be increased by increasing the HRP concentration and decreasing the hydrogen peroxide concentration, resulting in crosslinking within seconds [11]. This is a consequence of the fact that besides acting as a catalyst, $\mathrm{H}_{2} \mathrm{O}_{2}$ also deactivates HRP in high concentrations [11]. The crosslinking approach also proved to be non-cytotoxic despite the use of low quantities of $\mathrm{H}_{2} \mathrm{O}_{2}$ and allowed for encapsulation of $\mathrm{L} 929$ cells or Bone Marrow Stromal Cells (BMSCs) with over $95 \%$ viability $[11,122]$. A drawback of this approach is that this reaction is also prone to oxygen inhibition [123]. Recently, studies also indicated the potential towards photo-crosslinking of phenolic hydroxyl moieties, thereby clearing the road towards light-based additive manufacturing technologies [124] (Table 1).

Alternatively, transglutaminase can be used to crosslink gelatin without the prior need of a chemical modification [18]. Transglutaminase can catalyse the reaction between the gamma-carbonyl group of 
glutamine and the epsilon-amino group of a lysine amino acid resulting in the formation of an amide bond [18].

\section{Overview of Applied Photoinitiators}

When using light-based crosslinking chemistries, typically a photo-initiator is required to initiate the crosslinking reaction. Since gelatin is a hydrogel material, suitable photo-initiators need to be watersoluble, thereby rendering the options limited. A non-exhaustive overview of photo-initiators applied for gelatin crosslinking is presented below, which can be classified according to their activation behaviour.

\section{1. $\quad$ Norrish Type I photo-initiators}

Norrish type I photo-initiators are typically characterized by photocleavage into different smaller molecule radical species [125]. This type of PI is usually active in the UV-region and rarely exhibits activity in the visible spectrum. The most commonly applied photo-initiator for crosslinking and biofabrication purposes of gelatin derivatives is Irgacure 2959 (2-hydroxy-1-(4-(hydroxyethoxy)phenyl)-2-methyl-1-propanone) which is most efficient at $254 \mathrm{~nm}$ (Figure 4). This water-soluble photoinitiator has been considered as one of the gold standards since it has been commercially available for a long time [82]. Furthermore, at low concentrations (below $2.24 \mathrm{mM}$, corresponding to $0.05 \mathrm{wt} \%$ ), it is considered highly biocompatible which makes it suitable for biological applications $[28,62]$. Cytotoxic effects have been reported at concentrations exceeding $0.1 \mathrm{wt} \%$ corresponding to around 4.46 mM [62]. Since short wavelength (UV) irradiation has a low penetration depth and can induce cellular damage, research is shifting towards visible light initiators $[17,62,82,126]$. Unfortunately, Irgacure 2959 has proven to be very inefficient in the UV-A to visible light spectrum (i.e. molar absorptivity at $365 \mathrm{~nm}: 4 \mathrm{M}^{-1} \mathrm{~cm}^{-1}$ [2]). Therefore, researchers are increasingly substituting Irgacure 2959 by alternative photo-initiating systems. In this respect, lithium phenyl-2,4,6trimethylbenzoylphosphinate (LAP or Li-TPO-L) (Figure 4) has witnessed an increased use due to its higher efficiency in the visual spectrum (i.e. molar absorptivity at $365 \mathrm{~nm}: 218 \mathrm{M}^{-1} \mathrm{~cm}^{-1}$ [17]; at 400 $\mathrm{nm}: 30 \mathrm{M}^{-1} \mathrm{~cm}^{-1}[17]$; at $\left.405 \mathrm{~nm}: 50 \mathrm{M}^{-1} \mathrm{~cm}^{-1}[2]\right)$ and its superior water solubility while exhibiting a comparable biocompatibility to Irgacure $2959[17,28]$. Alternatively, also the use of VA-086 has been 
reported. VA-086 is an azo initiator resulting in the formation of nitrogen gas upon irradiation leading to the formation of gas bubbles in the hydrogel network (Figure 4) [27]. The photo-initiator is substantially less efficient in comparison to Irgacure 2959 as typically, a tenfold increase in concentration is required to yield comparable hydrogel properties [27]. However, Billiet et al. indicated an increase in cell viability using hepatocarcinoma cells during cell encapsulation experiments even at these high concentrations.

A final important class of PI's are 2PP-active PI's as 2PP gains increasing attention in the field of biofabrication. The first two-photon activated type I photo-initiator is DAS (tetrapotassium 4,4'-(1,2ethenediyl)bis(2-(3-sulfo-phenyl)diazenesulfonate)) which is a diazosulfonate-based initiator suitable for 2PP applications (vide infra) (Figure 4) [127]. However, DAS is not suitable to function as a conventional photo-initiator for linear absorption applications. Despite its lower activity in comparison to other 2PP-PI's at $800 \mathrm{~nm}$ as reflected by a lower two photon absorption cross-section (i.e. $40 \mathrm{GM}$ at $800 \mathrm{~nm}$ ), it is the only Type I photo-initiator suitable for cell encapsulation experiments during 2PP processing. Similar to VA-086, it is an azo initiator, implying that it produces nitrogen gas upon activation [127].

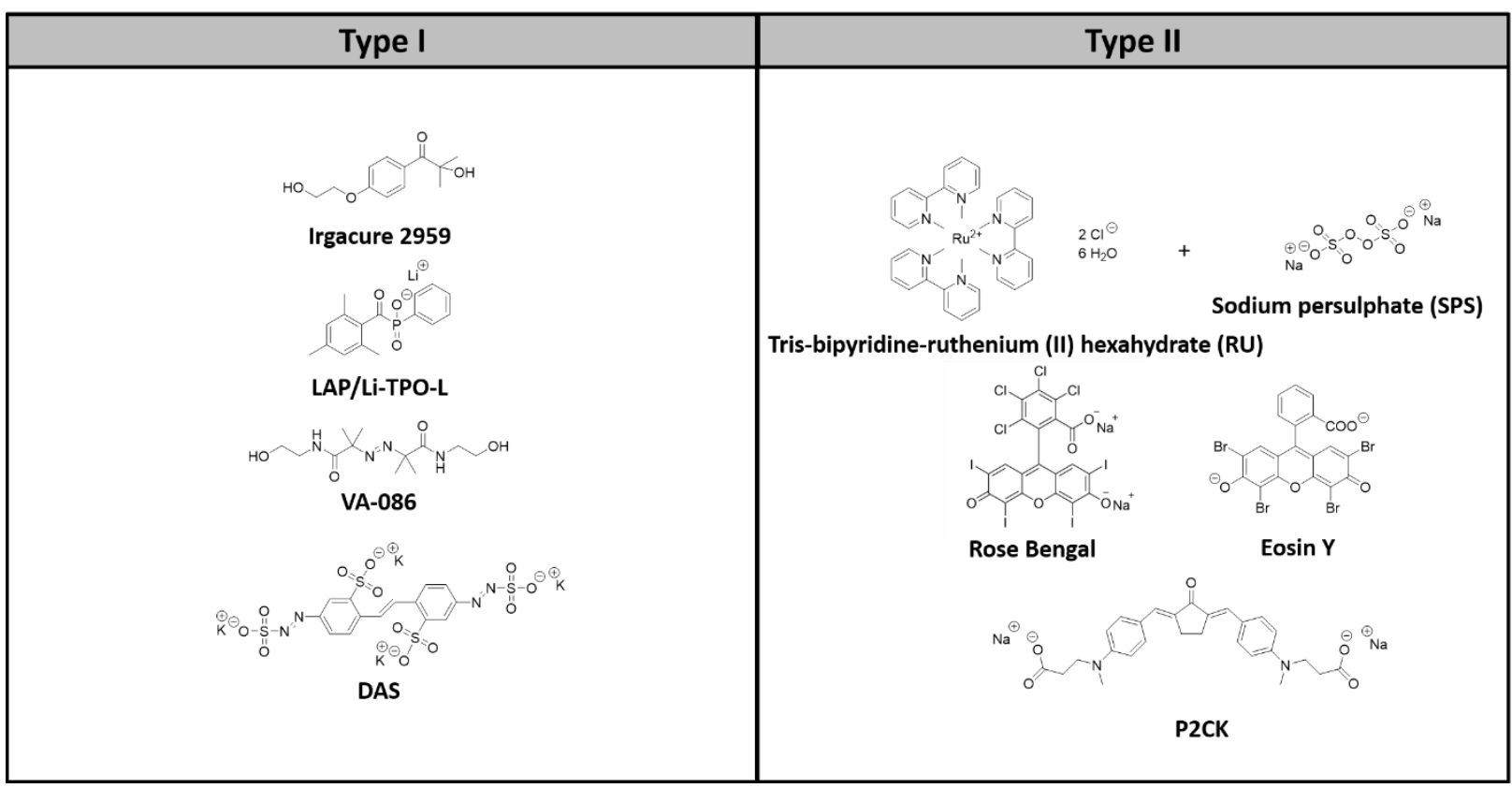


Figure 4: Overview of different photoinitiators applied for crosslinking of gelatin derivatives classified according to their initiaton mechanism.

\section{2. $\quad$ Norrish Type II Photo-initiators}

Type II initiators are initiators which can generate radicals without cleaving into smaller molecules [125]. Typical commercial examples of such molecules include eosin $Y$, rose bengal and ruthenium/SPS [17]. In contrast to type I PI's, most type II PI's require a (strong base) co-initiator (i.e. triethanolamine) to achieve a suitable reactivity for biofabrication approaches [24]. However, a lack of initiator reactivity can sometimes be overcome by using more reactive crosslinking chemistries. To this end, thiol-norbornene systems have for example shown to overcome the drawbacks associated with less efficient initiators [64]. Greene et al. have shown that eosin $Y$ can be applied to crosslink gelatinnorbornene with thiolated, 4-arm PEG in the absence of a co-initiator using visible light (i.e 550 $\mathrm{nm})$ [17]. Furthermore, they demonstrated its increased initiator efficiency, as 40 times more LAP was required to reach similar mechanical properties in comparison to eosin $Y$ at visible light irradiation (i.e. absorptivity of eosin $\mathrm{Y}: 100000 \mathrm{M}^{-1} \mathrm{~cm}^{-1}$ at $525 \mathrm{~nm}$ [17]). Additionally, eosin $\mathrm{Y}$ at $0.1 \mathrm{mM}$ exhibited an increased reactivity in comparison to LAP at $4 \mathrm{mM}$ as evidenced by a shorter gel-point time ( $24 \mathrm{~s}$ vs 42 s) [17]. Furthermore, eosin Y exhibits comparable cytotoxicity to LAP [17].

Another example of a type II visible light initiation system which is gaining increasing attention in the field of biofabrication is a ruthenium complex (tris-bipyridyl-ruthenium (II) hexahydrate) and sodium persulfate (SPS) as co-initiator and is referred to a $\mathrm{Ru} / \mathrm{SPS}[2,62,82,126]$. Upon irradiation, $\mathrm{Ru}^{2+}$ is photoexcited to $\mathrm{Ru}^{3+}$ followed by donating electrons to SPS, which in turn dissociates into sulphate anions and radicals $[82,128]$. Ru/SPS can be considered a very efficient $\mathrm{PI}$ as it is characterized by a high molar absorptivity: $14600 \mathrm{M}^{-1} \mathrm{~cm}^{-1}$ at $450 \mathrm{~nm}$ [2].

Finally, the most commonly applied PI to enable 2PP of hydrogel materials is also a type II initiator, namely P2CK (sodium 3,3'-(((1E,1E')-(2-oxocyclopentane-1,3-diylidene)bis(methanylydiebe))bis(4,1phenylene))bis(methylazanediyl))dipropanoate) $[16,29,64,129-131]$ which has proven to be very efficient (i.e. two photon absorption cross section: $140 \mathrm{GM}$ at $800 \mathrm{~nm}$ ) [127]. However, despite being a very efficient $2 \mathrm{PP} \mathrm{PI}, \mathrm{P} 2 \mathrm{CK}$ is not really suitable for cell encapsulation, as it can penetrate the cell 
membrane, whereafter upon irradiation, it will generate singlet oxygen, resulting in cytotoxicity making it an attractive candidate for two-photon based photodynamic therapy $[29,93,132]$.

\section{Important Considerations During Gelatin Modification Strategies}

Since gelatin is a biopolymer consisting of around 18 different amino acids with various functionalities in different ratios, the material is characterised by a specific behaviour towards solvents, reaction conditions, temperature, $\mathrm{pH}$, etc based on the relative amino acid composition.

Therefore, it is important to take a closer look at some of the aspects which are required to be taken into consideration when modifying or processing gelatin (-based) materials.

\subsection{Gelatin Functionalities Suitable for Chemical Modification and Analysis Thereof}

Gelatin contains a large number of functionalities in the side chains of the different amino acids which are prone to reaction. It contains amine functionalities in the side chains of lysine, hydroxylysine and ornithine (in gelatin type B), carboxylic acids in the side chains of glutamic and aspartic acid and hydroxyl functionalities in the side chains of serine, threonine and hydroxylysine [133]. Although most modification strategies discussed above use the primary amines as a handle to introduce functionalities, also the hydroxyl functionalities exhibit nucleophilic behaviour and can therefore compete in these reactions [69]. Depending on the reaction conditions, reports show that either both functionalities participate in the reaction or only one. Recently, Claassen et al. reported that during the modification of gelatin into gelatin methacryloyl (gel-MA) (Figure $1 \mathrm{~A}$ ), the hydroxyl functionalities participate in the reaction when a tenfold excess of methacrylic anhydride is added, while this is not the case when only 2 equivalents are added [69].

Additionally, Van Hoorick et al. investigated the degree of substitution (DS) of gel-MA and gel-NB using NMR spectroscopy based on the signals of the introduced functionalities (i.e. methacrylamide or norbornene signals) using the amino acid composition of the applied gelatin. They compared the obtained results with a spectrometric indirect amine determination technique based on orthophthalic dialdehyde. Both techniques yielded comparable results in terms of amine DS, thereby indicating only modification of the primary amines upon adding 1 equivalent methacrylic anhydride 
and upon applying a reaction time of 1 hour or by adding 0.75 or 2 equivalents of 5 -norbornene-2carboxylic acid succinimidyl ester and reacting for 20 hours thereby confirming that only modification of the primary amines occurs when using low molar excesses of reagents enabling the introduction of functionalities as reported by Claassen et al. [64].

Furthermore, Garcia-astrain et al. reported on a comparable DS for the modification of gelatin with furfuryl glycidyl ether after 24 hours of reaction as obtained via NMR spectroscopy, based on the signals of the furan ring and through a spectroscopic amine determination assay. This proves that also during this reaction, the hydroxyl functionalities remained unaffected [61].

In contrast, Shuster et al. reported on the modification of both the primary amines (100\%) and the carboxylic acids (35\%) during modification with glycidyl methacrylate at a molar excess of 17.75 equivalents at $40^{\circ} \mathrm{C}$ during an overnight reaction [134].

Bertlein et al. investigated the predominant site of modification during the functionalization of gelatin with allyl glycidyl ether. To this end, they calculated the number of reacted amines indirectly using a TNBSA (2,4,6-trinitrobenzene sulfonic acid) assay and compared this result with the DS as obtained via ${ }^{1} \mathrm{H}$-NMR spectroscopy. Furthermore, they looked into the reactivity of other functional groups by reacting model compounds including poly(allylamine), poly(vinyl alcohol) (PVA) and poly(acrylic acid) under the same reaction conditions. From this experiment, they observed that only $13 \%$ of the hydroxyl groups in PVA and only $10.8 \%$ of the carboxylic acids were reacted whereas full conversion was obtained for poly(allylamine) indicating the primary amines as the primary sites of reaction. However, an overestimation of the amount of introduced functionalities via NMR spectroscopy relative to the TNBSA assay was obtained when performing the modification under strong basic conditions, which is anticipated to be a consequence of the formation of additional primary amines due to basic hydrolysis of the amide functions along the backbone as further substantiated by GPC measurements [62]. Therefore, it can be concluded that indeed the hydroxyl functionalities can participate in the reactions targeting primary amine functionalization. However, this will only lead to 
a significant contribution when using large molar excesses of the applied reagent and prolonged reaction times (i.e. more drastic reaction conditions).

\subsection{Influence of modification on triple helix formation}

As already briefly discussed, gelatin is primarily composed of lysine, proline and hydroxyproline, where proline and hydroxyproline are responsible for the formation of hydrogen bonds resulting in triple helix formation $[7,133]$. Upon cooling below the dissociation/denaturation or gel temperature, the random coils in the gelatin solution start to aggregate to form microcrystalline junction zones resulting in physical crosslinks and therefore gel formation [6]. Reports have shown that introducing (bulky) groups (i.e. acyl groups) to the side chains of gelatin can hamper the triple helix formation and the associated renaturation properties associated to the physical gelation of gelatin (Figure $1 \mathrm{~B}, \mathrm{D}, \mathrm{C}, \mathrm{M}$ ) $[16,58,61,100,135]$. Since the transition from helix to coil is accompanied by endothermal heat, the effect of a modification on triple helix formation and associated physical gelation properties can be quantified using differential scanning calorimetry (DSC). These measurements can either be performed in solution or in the gel state during which the associated energies related to the physical gelation around $30^{\circ} \mathrm{C}$ can be assessed $[16,41,136]$. Alternatively, also strategies were reported in which the sample is first dehydrated by heating above $100^{\circ} \mathrm{C}$ followed by rapid cooling to prevent renaturation and by performing a second heating run during which differences in the glass transition $(\mathrm{Tg})$, which is associated to the triple helix formation of gelatin, can be compared $[47,58,61]$.

More recently, another approach to assess the triple helix formation has been elaborated which involves the use of modulated temperature DSC. In modulated DSC experiments, complex and overlapping thermal effects can be distinguished by superimposing a sinusoidal wave on the linear heating ramp. The resulting signal can be subdivided into a non-reversing and a reversing component via a deconvolution procedure $[6,137]$. As a consequence, time-dependent processes such as triple helix dissociation are present in the non-reversing signal whereas specific heat changes are visible in the reversing signal. Therefore, a straightforward distinction between the dissociation temperature $\left(T_{d}\right)$ and the $T g$ becomes possible[6]. By performing this method, Steyaert et al. indicated that the $\mathrm{Tg}$ 
decreases from about 104 down to $10{ }^{\circ} \mathrm{C}$ with moisture levels increasing from 0 to $23 \mathrm{wt} \%$. Furthermore, this $\mathrm{Tg}$ completely disappears in hydrogels, in contrast to triple helices which remain present both in the dry as in the hydrogel state and decrease with increasing water content since water acts as a plasticizer in between the gelatin chains [6]. However, at high water content, which is typically encountered in hydrogels, a plateau is obtained around $30^{\circ} \mathrm{C}[6]$.

At high modification degrees, typically observed when besides the primary amines, also other functionalities are modified, the physical gelation is no longer apparent, rendering mammal origin gelatin accessible to light-based additive manufacturing techniques including stereolithography $[5,16,138,139]$. In this respect, several research groups have attempted to render gelatin soluble at room temperature via the reaction of the carboxylic acids with ethane diamine (Figure $1 \mathrm{~J}$ ) [5], 2aminoethylmethacrylate (Figure 1 B) [16], dopamine (Figure 1 C) [36] or through acetylation of the hydroxyl groups (Figure 1 D) $[138,140]$. Another approach is the partial hydrolysis of gelatin, as it is known that the $T_{d}$ decreases with decreasing molecular weight $[7,43,134]$.

Bertlein et al. reported on the absence of physical gelation of gel-AGE based on porcine skin gelatin A at room temperature due to the modification of the primary amines with allyl glycidyl ether in alkaline conditions (Figure $1 \theta$ ) resulting in partial hydrolysis of the gelatin, thereby rendering it suitable for SLA applications [62]. However, when less basic conditions were applied during the synthesis of gelAGE, the material did maintain its physical gelation behaviour at room temperature [62].

Alternatively, when aiming at light-based additive manufacturing, the physical gelation behaviour of gelatin can be overcome by using gelatin originating from cold water fish which is already soluble at room temperature due to the lower concentrations of proline and hydroxyproline present within the backbone [141-143].

Finally, drastically increasing the surface to volume ratio for gelatin by electrospinning also results in cold water solubility. However, it should be noted that although the material becomes cold water soluble, it forms a hydrogel again within minutes [6]. 


\section{3. $\quad$ Degradation}

\subsubsection{Degradation of the Gelatin Backbone}

Gelatin is a material which is composed of robust amide bonds and will therefore not degrade under physiological conditions when crosslinked [60]. However, the material can be degraded by specific enzymes present in the natural ECM including collagenase, also referred to as matrix metalloproteinase 1 (MMP-1), which cleaves sequences such as Gly-Pro-Gln-Gly-lle-Ala-Gly-Gln between Gly and Ile, resulting in complete material degradation $[16,17]$. This effect was further substantiated by Koshy et al. who observed cell stretching of encapsulated 3 T3 fibroblasts in an inverse electron demand Diels-Alder crosslinked system at low gelatin concentrations due to matrix remodelling occurring by the cells. In a control experiment where the cells were treated with marimastat, which is an MMP inhibitor, the cells did not exhibit this stretching behaviour, thereby proving the enzymatic degradability of the material [100].

Another enzyme which allows gelatin degradation is chymotrypsin, which cleaves C-terminal peptide bonds preceding large hydrophobic amino acids (i.e. Tryptophan, proline and tyrosine) present in gelatin [14].

The crosslinking density of the material has a crucial effect on the degradation process $[16,60]$. Materials with a low crosslink density exhibit a linear correlation between mass loss and time indicating a surface erosion mechanism $[16,17]$. Besides longer degradation times, highly crosslinked gels typically first exhibit an increase in mass (in the hydrated state) due to cleavage of some crosslinks, while maintaining structural integrity resulting in an increased water uptake capacity. Only after longer degradation times, the materials start exhibiting a linear decrease in mass as a consequence of degradation $[14,16,17,24,60]$. Therefore, highly crosslinked gelatin materials exhibit a combination of bulk degradation and surface erosion behaviour [14].

The degradation mechanism can be of crucial importance when using gelatin hydrogels for drug delivery or growth factor as it will influence the release profile [14]. 
Furthermore, numerous studies indicate that the gelatin hydrogels degrade in the presence of cells as evidenced by differences in swelling ratio or storage moduli during culture [14]. Greene et al. observed a decrease in storage modulus over 14 days of culture in the presence of hepatocytic carcinoma cells, while the gels (gel-NB/PEG4SH 1-7wt\%) remained intact over the course of 14 days [14].

In order to prove the difference in network type between gelatin hydrogels crosslinked via a chain growth polymerization mechanism (gel-MA) and a step growth polymerization mechanism (gel-AGE + DTT), Bertlein et al. performed a partial collagenase degradation assay, by carrying out an acidic hydrolysis treatment followed by GPC analysis of the hydrolysis products. They observed the presence of non-degradable higher molecular weight fractions in the gel-MA hydrogels in comparison to the gel-AGE gels, as a consequence of the presence of the kinetic poly(methacrylamide chains) which are typically present in chain-growth hydrogels as discussed above [62].

\subsubsection{Degradation of Introduced Crosslinks}

The degradation behaviour of crosslinked gels can be tuned by selecting appropriate crosslinkers. For example when using ester-containing crosslinkers (i.e. PEGDA), hydrogels will degrade relatively fast even in the absence of enzymes. Conversely, when using a crosslinker with a more robust functionality (i.e. PEGDVS) degradation will only occur in the presence of enzymes [52]. As a consequence, the gelatin hydrogel composition can be tailored to tune the degradation properties, in particular when using multicomponent step-growth based systems. Some crosslinking chemistries focus specifically on the introduction of reversible crosslinks which can be cleaved when subjected to an external trigger including light resulting in spatiotemporal control over the degradation (Section 2.2.4.) or chemical triggers (Section 2.2.3.2. and Section 2.2.4.).

\section{Controlling the Mechanical Properties of Crosslinked Gelatin Hydrogels}

The mechanical properties of photo-crosslinkable gelatin hydrogels can be tuned in various ways either during gelatin modification or during material processing. 


\subsection{Influencing the Mechanical Properties by Chemical Modifications of Gelatin}

By varying the number of introduced crosslinkable functionalities, the mechanical properties of the crosslinked hydrogel can be controlled $[8,16,24,140]$. For most derivatives, the number of reacted primary amines (i.e. DS) can be controlled by varying the molar ratio of the functionalizing reagent (e.g. methacrylic anhydride [8], carbic anhydride [65], 5-norbornene-2-carboxylic acid [64], ...) with respect to the primary amines present in gelatin. When all primary amines are converted into crosslinkable functionalities, the mechanical properties can be increased even further by subsequent modification of the carboxylic acids present in the side chains of aspartic acid and glutamic acid with additional crosslinkable functionalities (e.g. 2-aminoethyl methacrylate) [16]. As a consequence, up to 5 times stiffer hydrogels can be obtained [16]. However, it should be noted that increasing the degree of modification will not always lead to an increase in hydrogel stiffness. Indeed, the introduction of additional functionalities can hamper triple helix formation (vide supra). Covalently crosslinked gels prove to be stronger when crosslinking occurs after physical gelation, since the triple helices formed can be 'locked' resulting in a smaller mesh size and associated superior mechanical properties $[16,140,144]$ (Figure 2 D). Alternatively, the mechanical properties of gelatin-methacrylamide can be altered through covalent linking to another biopolymer (e.g. alginate) prior to crosslinking [13]. Although weaker hydrogel blends have been reported compared to gelatin-methacrylamide, the modification enabled to fine-tune the mechanical properties through incorporation of divalent cations to physically crosslink the alginate chains [13]. An additional benefit of this approach is the formation of a network containing both protein and polysaccharide chains, thereby resulting in a more accurate ECM mimic with respect to the chemical composition [13]. Further approaches focussing on the formation of a superior ECM mimic comprising both proteins and glycosaminoglycans (GAGs) include the use of thiolated hyaluronic acid to crosslink gel-NB [24], the introduction of heparin onto gel-NB prior to crosslinking via coupling of the primary amines of gel-NB to the carboxylic acids in heparin using carbodiimide chemistry [14]. The introduction of heparin poses the additional benefit that it has specific domains to bind growth factors including VEGF and hepatocyte growth factor $[4,14]$. 
Furthermore, heparin even provides a stabilizing effect on growth factors as it protects them from denaturation and proteolytic degradation [4]. The incorporation of heparin loaded with hepatocyte growth factor in gel-NB gels resulted in the establishment of increased hepatocyte-specific functions for hepatocellular carcinoma cells (Huh7) [14].

\subsection{Influencing the Mechanical Properties During Hydrogel Processing}

After the introduction of crosslinkable functionalities onto gelatin, there are several methods to influence the mechanical properties of the hydrogel construct during processing. The most straightforward approach to vary the mechanical properties of a hydrogel is to vary the applied gelatin concentration for which higher concentrations typically lead to stiffer gels (Figure 5 A) $[8,16,24,38,64,71,89,145,146]$. However, evidence suggests that high gelatin concentrations $(>15$ $\mathrm{w} / \mathrm{v} \%)$ can compromise the biocompatibility and cellular response due to the presence of a too densely crosslinked network $[38,71,147]$.

It should be noted that if the mechanical properties are altered by altering the crosslinkable gelatin content, also the concentration of RGD functionalities alters and therefore, the biological response cannot only be attributed to the stiffness of the gel [14].

One way to overcome this limitation is through co-crosslinking gelatin with another photocrosslinkable material which can either be natural (e.g. polysaccharide) or synthetic (e.g. poly(ethylene glycol) (PEG) $[14,24,65,130,148]$. This co-crosslinking approach can be applied for chain-growth as well as step-growth polymerization systems. However, due to the high degree of supramolecular interactions occurring between the gelatin chains, which result in triple helix formation, the use of a secondary co-crosslinking material can result in phase separation as the secondary material can be excluded during physical gelation [12,149]. For example, Van Nieuwenhove et al. observed the formation of starch granules when co-crosslinking gel-MA with starch-pentenoate [12].

Examples of co-crosslinked materials using a chain-growth approach include k-carrageenan-MA [148], Starch-pentenoate (using DTT, via a step growth concurrent approach) [12], PEGDA [130,150], PEGTA [151], PVA-MA [2], HA-MA [67], pentaerythritol triacrylate (PTA) [43], trimethylolpropane triacrylate 
(TTA) [43], diisobutylacrylamide (DBA) [43], urethane-dimethacrylate (UDMA) [43], tripropylene glycol diacrylate (P3-A) [43], dipentaerythritol pentaacrylate (PPA) [43].

For step-growth polymerization of materials, a crosslinker is required which has a great influence on the mechanical properties of the resulting hydrogel. In this respect, especially the number of crosslinkable groups per molecule is of crucial importance [24]. For example, the use of a 4-arm PEGSH in comparison to a bifunctional DTT results in increased mechanical properties [65]. Additionally, Shih et al. compared the use of 4-arm PEGNB (20 kDa) with PEG-dinorbornene (10 kDa) to crosslink thiolated PVA hydrogels which resulted in an almost doubling of the hydrogel stiffness [24].

Examples of different applied crosslinkers in thiol-ene systems in which gelatin contains the -ene functionality (e.g. norbornene, allylether, pentenoyl, etc.) include DTT [64,65], PEG4SH 10kDa $[14,17,65]$, thiolated polyvinylalcohol (TPVA) [24], thiolated hyaluronic acid (THA) [24,52], thiolated gelatin $[46,53]$.

Examples of applied crosslinkers in thiol-ene systems in which gelatin contains the thiol functionality include PEGDA [49,52], hyperbranched acrylated PEG [89], pentenoyl gelatin [53] , PEG-divinylsulfone (PEGDVS) [52], gel-NB [46].

In some cases, additional co-crosslinked materials can be introduced besides the crosslinker to influence the mechanical properties of the hydrogel. For example, Greene et al. applied PEG4NB together with a gel-NB/PEG4SH system resulting in a 10 -fold increase in storage modulus (i.e. $0.8 \mathrm{kPa}$ up to $8 \mathrm{kPa}$ ) when incorporating up to $1.68 \mathrm{wt} \%$ PEG4NB into 2 wt\% gel-NB/PEG4SH gels without varying the biologically active component (i.e. gelatin)[14]. Shih et al. added PEG4SH to a gel-NB/gelSH system to increase the mechanical properties without increasing the total gelatin concentration in the mixture [24]. Examples of co-crosslinked materials which do not function as crosslinker in thiolene systems include PEG4NB 20 kDa [14] , PEG-dinorbornene 10 kDA (PEGdNB) [24] and thiolated-HA [52]. 
Furthermore, Greene et al. observed that the presence of low gelatin concentrations (i.e. 1 - 3 wt\% gel-NB) in crosslinked gel-NB/PEG4SH gels resulted in lower cell survival of encapsulated Huh7 hepatic carcinoma cells in comparison to higher concentrations (i.e. 5 - 7 wt\%) [14].

When using the same gelatin content, but varying the stiffness of the gel by varying the thiol-ene ratio in gel-NB gels, it was shown that hepatocyte cells exhibit a higher metabolic activity in gels with lower stiffness for an identical gelatin content [14].

Thiol-ene systems have another benefit over chain-growth hydrogels in the sense that by varying the thiol-ene ratio, the gelatin content can be tuned without changing the network density nor the associated mechanical properties $[14,24,64,65,81]$. To this end, Greene et al. managed to vary the gelNB content from 1 to $7 \mathrm{wt} \%$ while keeping the thiolated crosslinker concentration constant, resulting in similar mechanical properties throughout the complete concentration range [14].

Another handle to tune the mechanical properties of the crosslinked network is a variation in the applied irradiation dose for crosslinking $[9,14,16,27,38,145,146]$. Generally, lower doses result in weaker hydrogels as lower crosslink densities are obtained $[14,27,38]$. However, influencing the mechanical properties by varying the irradiation dose also affects the number of unreacted, potentially cytotoxic functionalities. Additionally, when chain-growth hydrogels are applied, varying the irradiation dose often is concomitant with a reduced reproducibility due to the complex reaction kinetic profile in combination with oxygen inhibition occurring during crosslinking [81]. Furthermore, when using highly reactive thiol-ene systems (e.g. norbornene), the influence of the dose will be less apparent, since already at very low irradiation doses (during 2PP: $20 \mathrm{~mW}$ at $100 \mathrm{~mm} / \mathrm{s}$ for gel-NB with a fully crosslinked network from $40 \mathrm{~mW}$ onwards vs $\geq 80 \mathrm{~mW}$ for gel-MA with a clear correlation between irradiation energy and swelling degree), the material will fully crosslink [16,64].

A final strategy is combining gelatin hydrogels with other materials (e.g. polyesters) for their mechanical properties without covalent linking $[68,152]$. This can either be done by combining it with a stiff scaffolding material (e.g. polyesters) obtained either via macro- or microprinting[28,31,68,152]. For example, Visser et al. managed to drastically improve the mechanical properties of gelatin via the 
incorporation of PCL fibres produced via melt electrowriting resulting in a stronger scaffold in comparison to the pure PCL scaffold or a pure gelatin pellet [68]. Markovic et al. applied a PLA scaffold obtained via fused deposition modelling and introduced a gel-MA bioink as an ECM mimic containing pre-osteoblasts inside in order to benefit from the stiff PLA for mechanical properties [28]. 

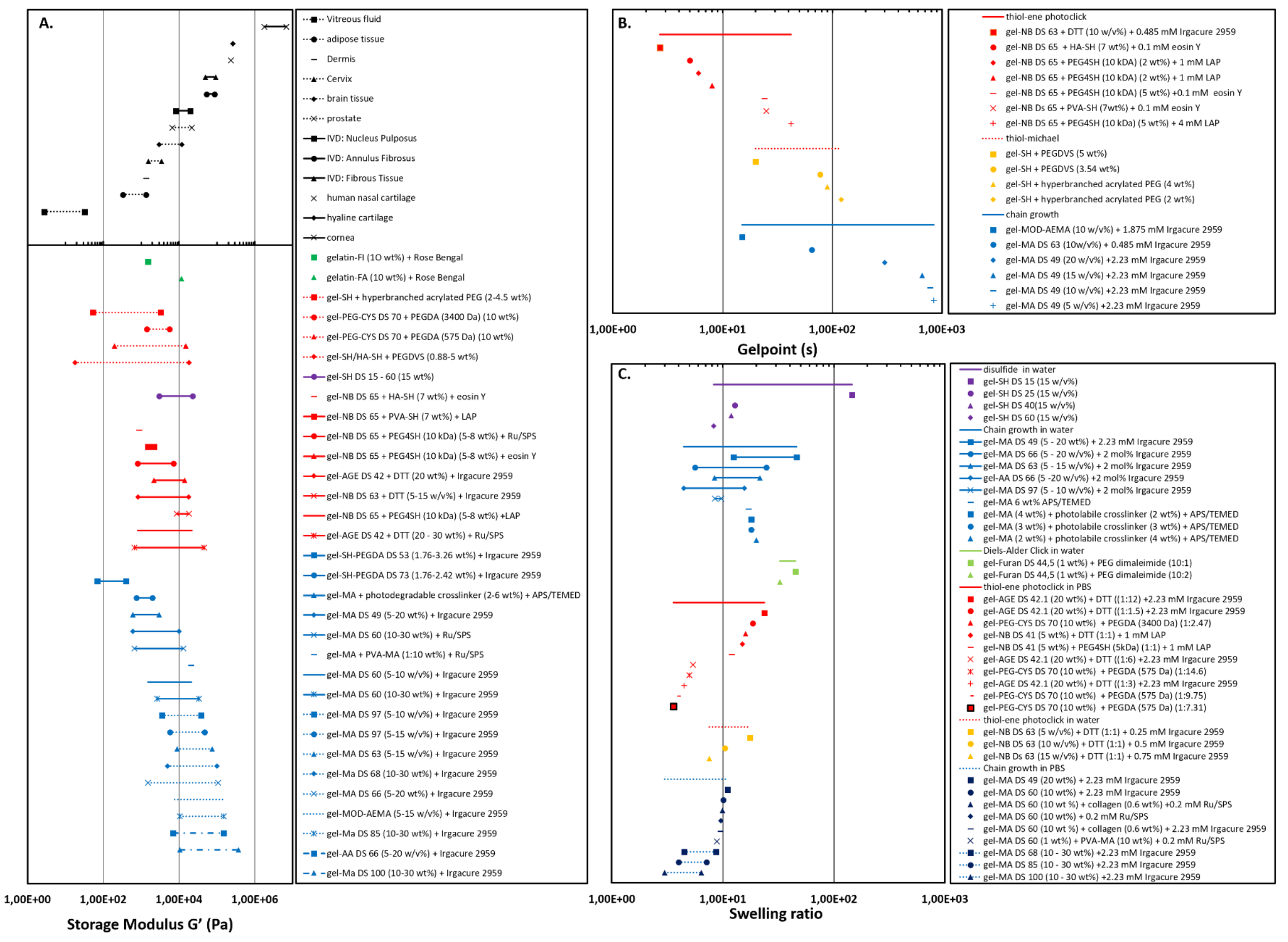
Figure 5: Scheme representing the physico-chemical properties of reported gelatin hydrogels (A.) .Storage moduli of reported gelatin derivatives classified according to crosslinking mechanism including photooxidation (green): gelatin-FI and gelatin-FA [54]; Thiol-Michael addition (red, dashed): gel-SH + hyperbranched PEG [89], gel-PEG-Cys [49]; gel-SH/HA-SH + PEGDVS [52]; Disulphide formation (purple): gel-SH [45]; Thiol-ene photoclick (Red, solid): gel-NB DS 65 + HA-SH [24], gel-NB DS 65 + PVA-SH [24], gel-NB DS 65 + PEG4SH (10 kDa) [17], gel-AGE DS 42 [62], gel-NB DS 63 [64]; Chain growth (Blue): gel-SH-PEGDA [44], gel-MA + photodegradable crosslinker [117], gel-MA DS 49 [153]; gel-MA DS 60 + Ru/SPS [62], gel-MA + PVA-MA [2], gel-MA DS 60 (5-10 w/v\%) [8], gel-MA DS 60 (10-30 wt\%) [62], gel-MA DS 97 [8,16], gel-MA DS 63 [64], gel-MA DS 68 [101], gel-MA DS 66 [38], gel-MOD-AEMA [16], Gel-MA DS 85 [101], gel-AA DS 66 [38], gel-MA DS 65 + CS-MA [154], gel-MA DS 100 [101] - in comparison to the mechanical properties of different tissues including: vitreous fluid [155], adipose tissue [156,157], dermis [158], cervix [159], brain tissue [160], prostate [161], intervertebral disc (IVD): nucleus pulposus [79], annulus fibrosus [79], fibrous tissue [79], human nasal cartilage [101,162], cornea [163].

(B.) Overview of reported gel points for different gelatin derivatives organized according to crosslinking mechanism including: thiol-ene photoclick (red): gel-NB DS 63 [64], gel-NB DS $65+$ HASH [24], gel-NB DS 65 + PEG4SH (10 kDa) [17], gel-NB + PVA-SH [24]; thiol-Michael (red dashed, orange): gel-SH + PEGDVS [52], gel-SH + hyperbranched acrylated PEG [89]; Chain-growth (blue): gel-MOD-AEMA [16], gel-MOD DS 63 [64], gel-MA DS 49 [153].

(C.) Mass swelling ratios of different reported gelatin derivatives organized according to crosslinking mechanism and applied solvent: disulphide in water (purple): gel-SH [45], chain growth in water (blue): gel-MA DS 49 [153], gel-MA DS 66 [38], gel-MA DS 63 [64], gel-AA DS 66 [38], gel-MA DS 97 [8], gel-MA 6 wt\% + photolabile crosslinker [117]; Diels-Alder click in water (green): gel-FA, gel-Fl [54]; thiol-ene photoclick in PBS (red): gel-AGE [54], gel-PEG-cys [49], gel-NB DS 41 [65]; thiol-ene photoclick in water (red dashed, orange): gel-NB DS 63 [64]; chain growth in PBS (dark/dashed blue): gel-MA DS 49 [153], gel-MA DS 60 [82], gel-MA DS 60 (1wt\%) + PVA-MA (10 wt\%) [2], gel-MA DS 68,85 \& 100 [101]

(If the elastic modulus $E^{\prime}$ was presented in the original document, an estimation of the shear storage modulus was obtained using $\left.E^{\prime}=2 G^{\prime}(1+\mu)\right)$ in which $\mu$ is 0.5 for ideal rubbery networks) [164]. 
All these aspects render gelatin hydrogels suitable to cover a broad range of mechanical properties. A non-exhaustive overview of the mechanical property range of earlier reported gelatin derivatives compared to the mechanical properties of different tissues can be found in Figure 5 [16]. As a consequence, gelatin-based materials prove to be versatile tools for mimicking the mechanical properties of a plethora of tissues.

\section{Gelatin Processing via Additive Manufacturing}

Gelatin-based hydrogels have frequently been processed via additive manufacturing (AM) techniques. These methods are mostly based on the layer-by-layer fabrication of 3D constructs according to a CAD model. Printing of the materials can either occur by following a direct approach during which the material is directly processed in the AM step [27] or by an indirect approach in which a template is fabricated using AM to control the shape of the secondary material $[8,165]$.

Indirect approaches are either applied to combine the mechanical properties of a stiff polymer with the desirable cell interactivity of gelatin or to introduce complex 3D structures into materials which cannot be processed through a direct AM method $[8,28,31,70,165]$. Van Hoorick et al. were able to produce self-supporting, low density (i.e. $5 \mathrm{w} / \mathrm{v} \%$ ) gel-MA scaffolds by using a sacrificial polyester scaffold [8]. Furthermore, besides a bio(material)ink, gelatin is often also used as a coating material to render scaffolds more cell interactive/cytocompatible $[70,166,167]$.

The direct additive manufacturing techniques applied for gelatin processing can be subdivided into 3 groups: (i) inkjet-based (ii) syringe-based and (iii) light-based techniques, including stereolithography (SLA), digital light processing (DLP) and 2PP. (Figure 6). 
a)

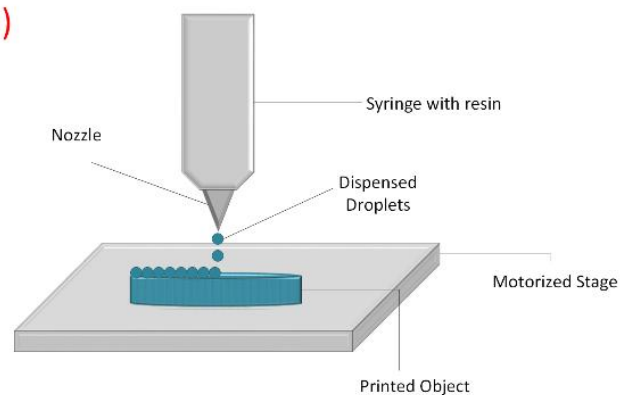

c)

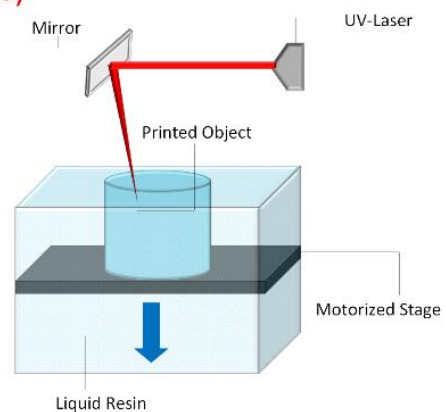

d)
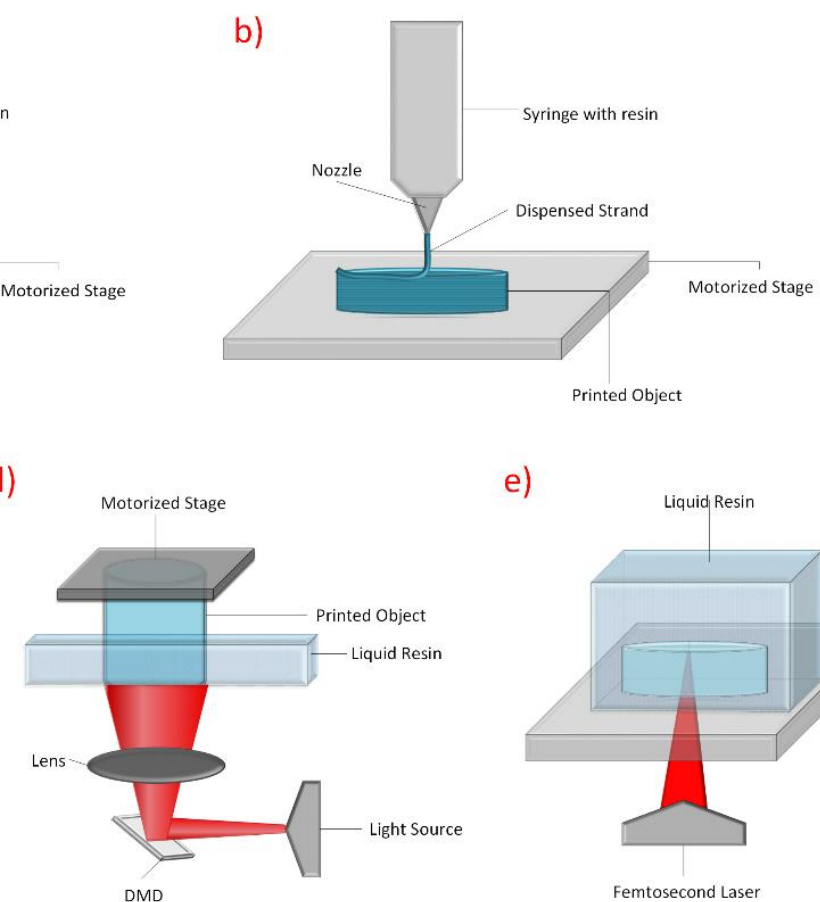

e)

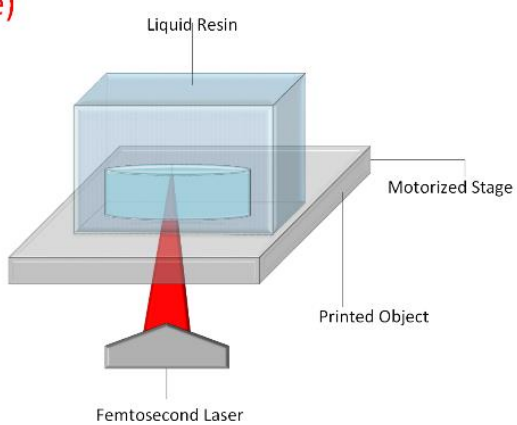

Figure 6. Overview of additive manufacturing techniques: a) Inkjet-based 3D printing, b) Syringe-based 3D printing, c) Stereolithography (SLA), d) Digital light processing (DLP) and e) Two-Photon Polymerization (2PP).

\subsection{Inkjet-Based 3D Printing}

An inkjet-based 3D printer is able to quickly generate $10-50 \mu \mathrm{m}$ sized droplets (i.e. picolitres) either through a thermal or a piezoelectric approach $[34,80]$. This technique has a low risk of contamination to occur due to the fact that it is a non-contact and drop-on-demand printing method [138]. Several studies have already shown that inkjet bioprinting can be successfully applied for processing of viable mammalian cells including neurons and endothelial cells $[168,169]$. However, in these studies, printing occurred in cell culture medium or physiological buffer without a matrix to support the cells. Therefore, Hoch et al. developed a double chemical functionalized gelatin derivative that can be used as bioink for piezoelectric, inkjet-based 3D printing (Figure 7). To this end, the authors methacrylated and acetylated both the primary amines and the carboxylic acid moieties of gelatin respectively to render it soluble at room temperature (see section 4.2). Irgacure 2959 was applied as photo-initiator in order to obtain a crosslinked hydrogel network. The cell viability of the encapsulated porcine chondrocytes close to $100 \%$, even after 240 min incubation. 

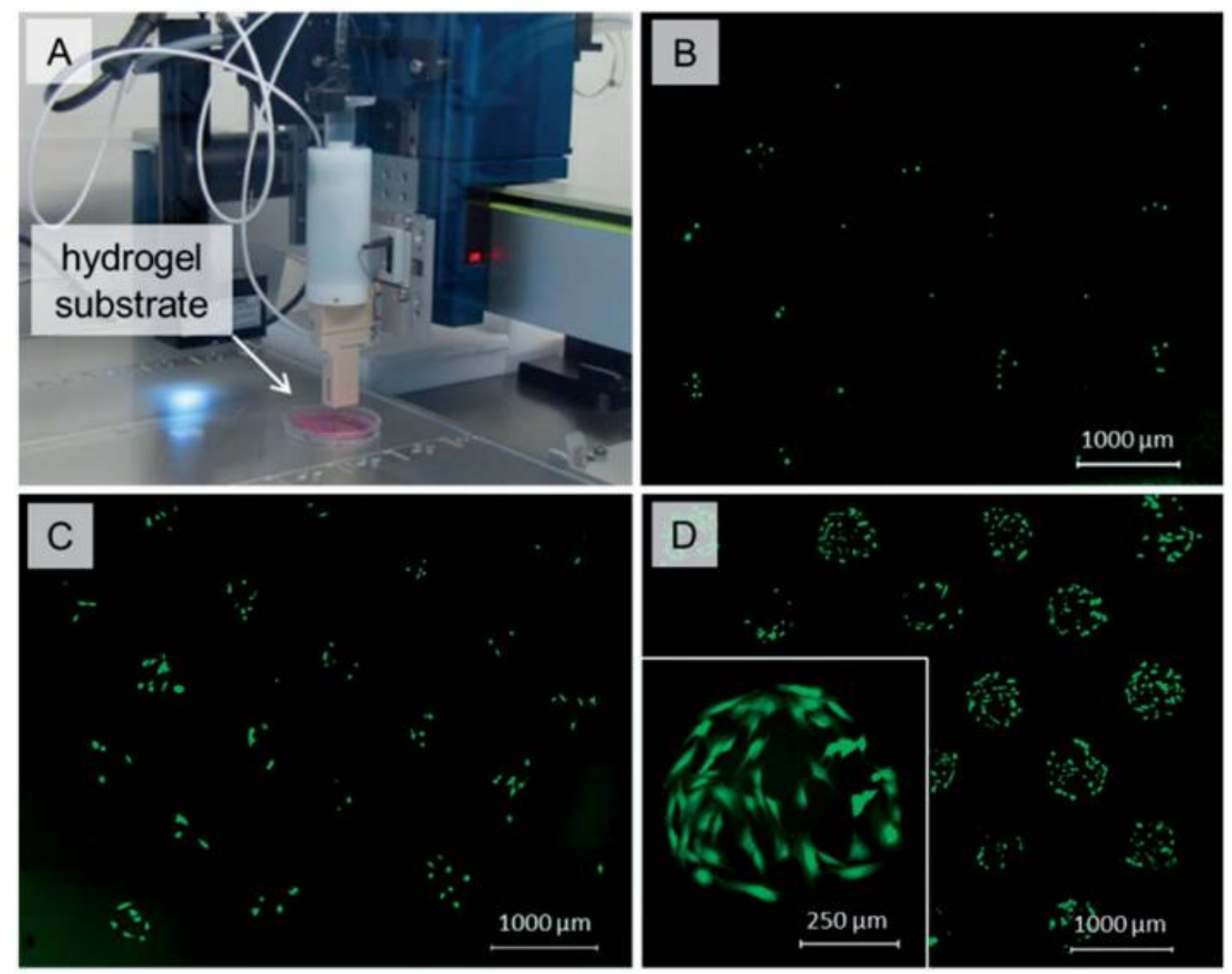

Figure 7: Piezoelectric printing of porcine chondrocytes. (A) The cells were suspended in a $10 \mathrm{wt} \%$ double modified gelatin-based bioink $\left(1 \times 10^{6}\right.$ cell per $\left.\mathrm{mL}\right)$ and were printed onto swollen gelatin-based substrates. Subsequently, the constructs were incubated in cell culture medium. The cell viability and morphology of the printed cells were determined using live/dead staining. (B) Images recorded 3h, (C) 24h, (D) $72 \mathrm{~h}$ after printing. Reproduced from Hoch et al. [138] with permission.

\subsection{Syringe-Based 3D Printing}

Probably the most widespread technique used for the fabrication of 3D scaffolds for tissue engineering applications is syringe-based 3D printing due to its simplicity, relatively low cost and its compatibility with a wide range of materials. In this approach, the material is either pneumatically or mechanically (piston- or screw-based) dispensed through a nozzle-based printing head. The strands of the material will be continuously extruded into 3D constructs by stacking $2 \mathrm{D}$ patterns according to a CAD model. Multiple parameters including the nozzle diameter, the writing speed, the applied pressure and the mechanical properties of the material will determine the spatial resolution of the 3D-printed structures [170]. Several literature reports have already stated that different gelatin derivatives can 
be used for syringe-based 3D printing applications $[27,34,148,171]$. One of the main advantages of this printing technique is that it enables the extrusion of the material both in the presence or absence of living cells. When a material is printed into a scaffold and is subsequently seeded with cells, the material is referred to as a biomaterial ink [172]. On the other hand, when a combination of biomaterials and cells is applied, it is commonly referred to as bioink [172].

Gelatin derivatives such as gel-MA are widely used as bioink due to their high cytocompatibility and tunable physical properties [173]. However, previous literature reports have indicated that several parameters including bioink concentration, printing pressure as well as nozzle type should be taken into account to produce cell-laden, gelatin-based 3D scaffolds with a high structural fidelity and a high cell viability using syringe-based bioprinting $[27,173,174]$.

Experimental results and computational fluid dynamics' simulations obtained by Billiet et al. and Liu et al. have shown that the nozzle type (conical versus straight) has an influence on the cell viability of encapsulated hepatocarcinoma cells (HepG2) and human umbilical vein endothelial cells (HUVECs) respectively in a gel-MA bioink $[27,173]$. The results indicated that high shear stresses, which are harmful for the cells, were generated along the entire length of a cylindrical, straight needle, while high shear stresses only existed at the very tip of conical nozzles resulting in higher cell viabilities. However, when high printing pressures are applied, cells experience high shear stresses in both types of nozzles [27].

Furthermore, cell-laden scaffolds with a high structural fidelity could be printed using gel-MA bioink concentrations between 10 and 20 w/v\% and Irgacure 2959 as photo-initiator [27,174]. The obtained high cell viability results (> 90\%) for the encapsulated HepG2 and primary human chondrocytes respectively indicated that the cells survived the printing process. However, it is known that high gelMA concentrations (> $10 \mathrm{w} / \mathrm{v} \%$ ) result in more densely crosslinked networks with a higher stiffness resulting in a decreased cell proliferation and migration rate for the encapsulated cells $[147,173,174]$. Therefore, lower concentrations of gel-MA bioinks would be more ideal for biofabrication purposes. Nonetheless, additively manufactured scaffolds using a low gel-MA concentration $(<7 \mathrm{w} / \mathrm{v} \%)$ 
exhibited low shape fidelity due to severe internal pore collapse [27]. Malda et al. reported this phenomenon as being one of the current paradoxes towards successful biofabrication of hydrogel materials [147]. Consequently, researchers are focussing on different strategies to circumvent the problems associated with low concentrated gel-MA bioinks in order to fabricate constructs with a high structural fidelity and a high cell activity using low gel-MA bioink concentrations. One possible solution would be to add sacrificial materials including unmodified gelatin, sodium alginate, gellan gum and hyaluronic acid to increase the viscosity of the gel-MA bioink facilitating syringe-based bioprinting $[146,151,175,176]$. However, it should be taken into account that the added sacrificial materials should not be toxic for the cells and should either degrade or carefully removed without destroying the structure of the scaffold. Yin et al. already combined gel-MA $(5 \mathrm{w} / \mathrm{v} \%)$ with gelatin $(8 \mathrm{w} / \mathrm{v} \%)$ and $0.5 \mathrm{w} / \mathrm{v} \% \mathrm{Li}-\mathrm{TPO}-\mathrm{L}$ to increase the viscosity of the bioink and to improve the reversible physical gelation properties of the bioink [175]. The authors successfully printed bone marrow-derived stem cell-laden (MSCs) scaffolds with a similar geometrical resolution compared to $30 \mathrm{w} / \mathrm{v} \%$ gel-MA scaffolds. The gelatin was gradually dissolved from the scaffold when the temperature was increased above $30^{\circ} \mathrm{C}$ without affecting the scaffold geometry. The obtained results indicated that the encapsulated cells were able to migrate and were still viable (> 90\%) after 7 days [175]. Furthermore, Jia et al. incorporated alginate in a bioink based on gel-MA, 4-arm poly(ethylene glycol)-tetra-acrylate (PEGTA) and Irgacure 2959 to enable fast ionic crosslinking and shape maintenance of the printed scaffold by co-delivery a $\mathrm{CaCl}_{2}$ solution prior to permanent fixation through UV-induced crosslinking of the gelMA and PEGTA precursors (Figure 8) [151]. Afterwards, the alginate was removed and scaffolds with a high cell viability ( $>80 \%$ ) were obtained [151]. 


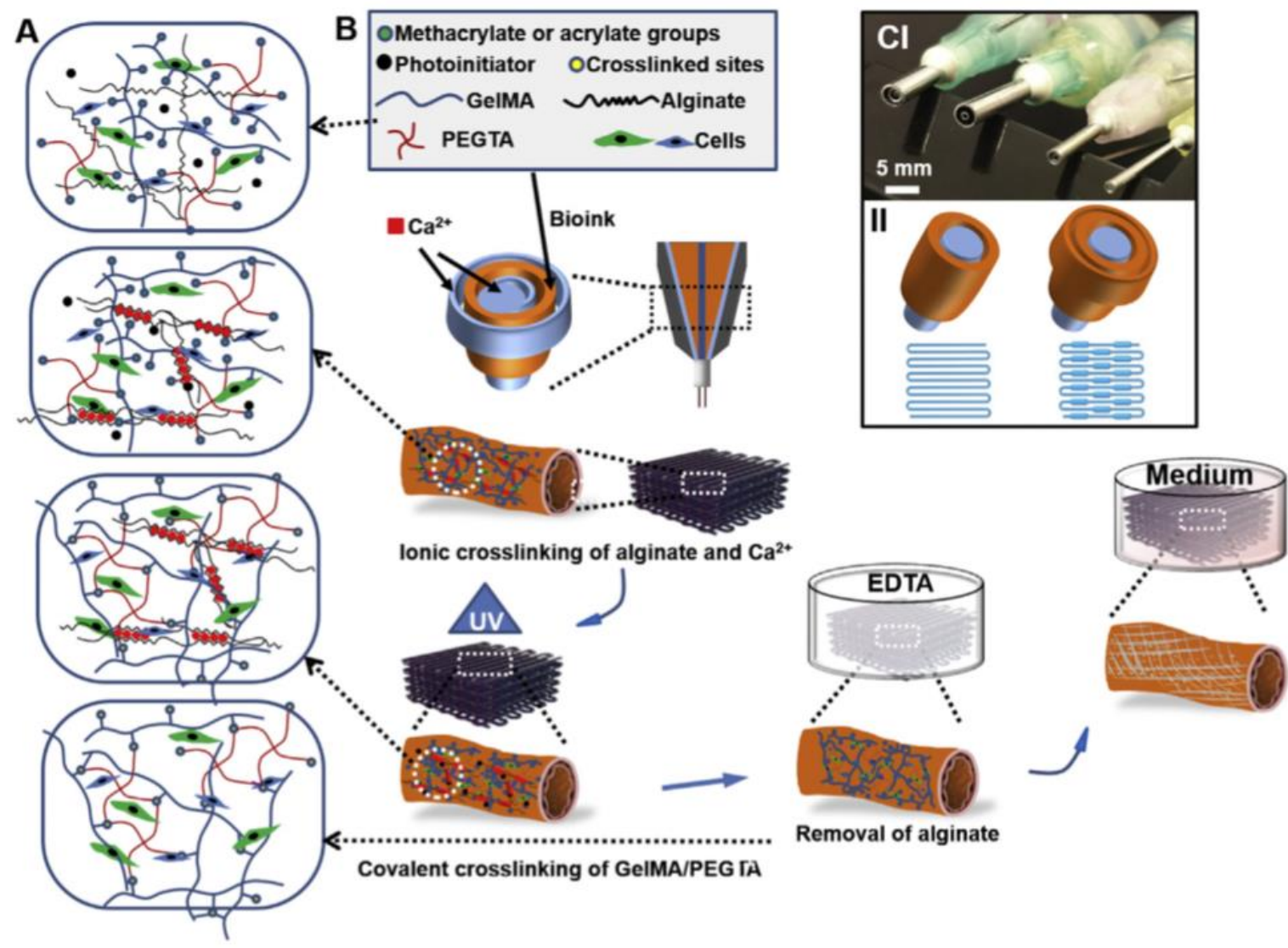

Figure 8: (A) Schematic illustration showing the two independent crosslinking processes of a bioink, in which alginate, gel-MA and PEGTA are ionically and covalently crosslinked, respectively, upon exposure to $\mathrm{CaCl}_{2}$ solution and UV light. (B) Schematic overview of the bioprinting of perfusable hollow tubes with the cell-encapsulating bioink and subsequent vascular formation. (C) Designed multilayered coaxial nozzles and schematic illustration showing fabrication of perfusable hollow tubes with constant diameters and changeable sizes. Reproduced from Jia et al. [151] with permission.

A second strategy is based on the pre-crosslinking of the bioink prior to syringe-based bioprinting or before the extruded strands were collected on the substrate. Levato et al. pre-crosslinked the gel-MA bioink for $10 \mathrm{~s}$ in the presence of Irgacure 2959 with UV-A light to improve the viscosity of the bioink [177]. However, pre-crosslinking typically resulted in high and inconsistent extrusion forces, heterogeneous 3D printed scaffolds and low cell viability [178]. In situ crosslinking would be a more promising alternative due to the low and consistent extrusion forces, uniformly printed strands and high cell viability of encapsulated cells. The advantages associated with this strategy include: (i) viscosity enhancement or co-polymerization with other polymers is not required, (ii) a wide range of photo-crosslinkable bioinks can be applied, (iii) the encapsulation of viable cells is allowed and (iv) 
complex and heterogeneous structures can be printed [178]. Indeed, Bertassoni et al. successfully applied the in situ crosslinking principle to fabricate HepG2 cell-laden 3D printed scaffolds with a high cell viability (> 80\%) [179]. A third option was investigated by Liu et al. who induced physical gelation in gel-MA by a straightforward cooling process down to $4{ }^{\circ} \mathrm{C}$ [173]. No sacrificial materials or precrosslinking steps were required to enable syringe-based bioprinting of 3-5 w/v\% gel-MA bioinks. The results indicated that scaffolds with a high shape and structural fidelity were produced due to the shear thinning and self-healing properties of the gel-MA physical gel [173]. Subsequently, the scaffolds were UV crosslinked in the presence of Irgacure 2959 to realize permanent stabilization. Furthermore, the research indicated that cooling of the gel-MA pre-bioinks in order to obtain a physical bioink did not exert a noticeably negative impact on the cell viability of the encapsulated HUVECs [173].

Natural and/or synthetic materials are often combined to create 3D scaffolds that closely mimic the physico-chemical characteristics of the in vivo environment of the ECM. Gelatin has already been combined with biopolymers including (modified) alginate and hyaluronic acid and/or synthetic materials such as PEGTA $[151,180-183]$. To date, methacrylated hyaluronic acid (HA-MA) has already been successfully applied in various biomedical applications. However, HA-MA is not cell-interactive and is therefore frequently combined with gel-MA. Indeed, Skardal et al. and Qi et al. printed stable, cell-laden 3D constructs with a bioink consisting of gel-MA and HA-MA combined with 2,2-dimethoxy2-phenylacetophenone or Irgacure 2959 respectively for tissue engineering purposes [181,183]. Furthermore, Ruther et al. and Colosi et al. combined alginate di-aldehyde with gelatin and alginate with gel-MA respectively for vascular tissue engineering applications following a Schiff's base crosslinking approach (vide supra) $[180,182]$. Synthetic materials are often added to gel-MA bioinks in order to increase the mechanical properties. Jia et al. for example combined gel-MA with a 4-arm PEGTA due to the superior crosslinking density and thus increased mechanical strength of PEGTA compared to linear PEG derivatives [151]. The latter is due to the 4-arm branched structure and multiple photo-crosslinkable sites resulting in the maintenance of a beneficial porous structure. 
The potential of bioinks based on other gelatin derivatives has also been investigated. Indeed, Yan et al. used a thiolated gelatin bioink combined with calcium ions and a homobifunctional maleimidepoly(ethylene glycol)-maleimide crosslinker for the fabrication of 3D scaffolds (Figure $1 \mathrm{~L}$ ) [184]. The obtained results indicated that long-term stable scaffolds were formed (> 1 month) and that the encapsulated SV40 immortalized mouse cholangiocytes survived the printing process resulting in a high cell viability [184]. Furthermore, Bertlein et al. developed a bioink based on allylated gelatin (gelAGE, Figure $1 \theta$ ) with DTT and Irgacure 2959 which was processable via syringe-based bioprinting. The encapsulated cells remained viable (> 70\%) after 23 days of cell culture [62].

The precise positioning/switching between different cell types remains a major challenge in tissue engineering [182]. However, the combination of bioprinting of a cell-laden bioink into a scaffold and post-seeding a different cell type on top of the printed construct holds great potential to produce scaffolds with co-cultured cells. Colosi et al. created HUVECs-laden gel-MA-alginate based scaffolds which were seeded afterwards with cardiomyocytes in order to investigate whether or not the construct could serve as an in vitro platform for cardiac tissue engineering applications [182]. The obtained results showed that the constructs were soft enough to enable the migration of the encapsulated HUVECS while being strong enough to support the synchronic beating of cardiomyocytes.

\subsection{Light-Induced Techniques}

Photo-crosslinkable gelatin derivatives are also frequently processed via light-induced techniques including stereolithography (SLA), digital light processing (DLP) and 2PP due to the advantages these techniques hold over syringe-based 3D printing [150,185-187]. First of all, clogging problems are eliminated, because these methods are nozzle-free. In addition, shear stresses due to the material passing through a nozzle-based printing head are avoided rendering this approach suitable for printing in the presence of living cells [188]. Furthermore, the printing speed and the resolution of lightinduced techniques are generally much higher compared to syringe-based methods [2]. Therefore, 
these techniques exhibit the potential to better mimic the complex and microscale architecture of biological tissues.

\subsubsection{Stereolithography}

Stereolithography (SLA) is a rapid prototyping technique that exploits a laser light beam, typically UV light, to polymerize photo-crosslinkable materials in a layer-by-layer scanning approach in order to fabricate complex 3D scaffolds with a high resolution (Figure 6A) [185]. Several research reports have already indicated that photo-crosslinkable gelatin derivatives such as gel-MA are processable via stereolithography $[36,150]$. Zhou et al. modified the primary amine functionalities of gelatin with methacrylamide groups and the carboxylic acid moieties with dopamine to obtain a photocrosslinkable gelatin derivative that was crosslinked in the presence of Irgacure 2959 (gelMA-DA Figure $1 \mathrm{C}$ ) suitable for neural tissue engineering (Figure 9) [36]. The developed scaffolds exhibited a homogeneous, highly porous and interconnected 3D environment which was favourable for supporting the growth and differentiation of the seeded neural stem cells [36]. Furthermore, the research of Zhu et al. showed that stereolithography can also be applied in the presence of living cells [150]. However, although the mechanical properties of gel-MA are ideal for soft tissue engineering, they prove to be insufficient for cartilage regeneration [150]. Therefore, the authors combined gelMA with the synthetic PEGDA and the photo-initiator Irgacure 2959 [150]. Moreover, the addition of a synthetic polymer not only increased the mechanical properties but also enhanced the printing resolution of the bioink. The results indicated that the compressive modulus could be increased from 1 up to $5 \mathrm{MPa}$ and that the width of a printed line, reflecting the attainable resolution, decreased from 950 to $350 \mu \mathrm{m}$ by adding $5 \mathrm{w} / \mathrm{v} \%$ PEGDA to $10 \mathrm{w} / \mathrm{v} \%$ gel-MA. In addition, a high viability (> 75\% at day 1) and a high proliferation rate were observed for the encapsulated MSCs in these scaffolds indicating that the synthetic component did not negatively affect the cells [150]. 


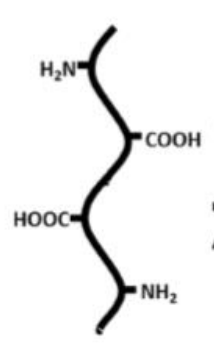

Gelatin

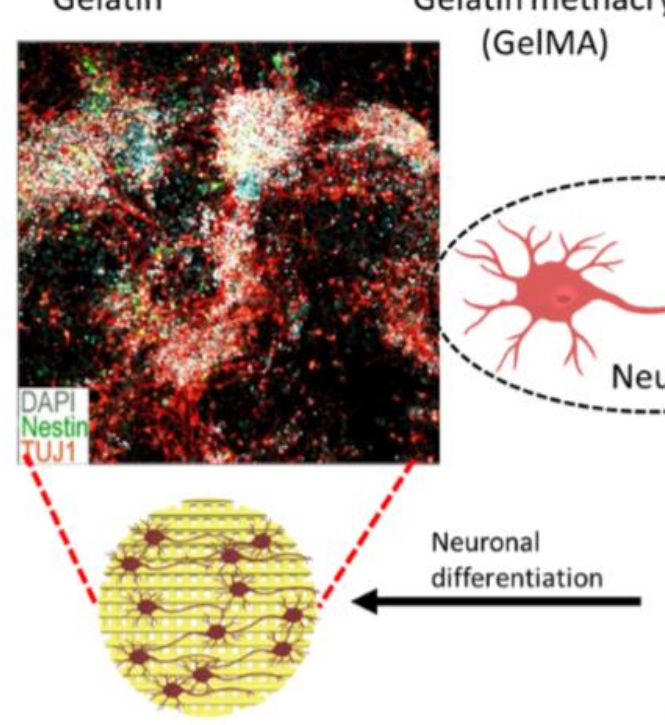

Neuronal network

(GelMA)

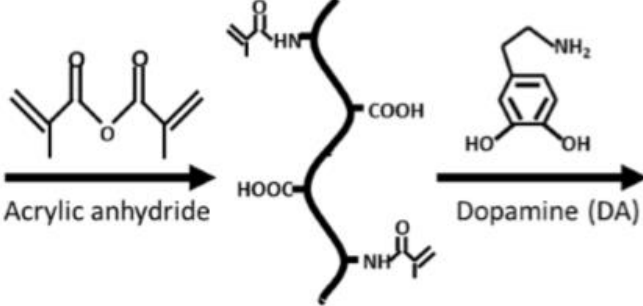

Gelatin methacrylate

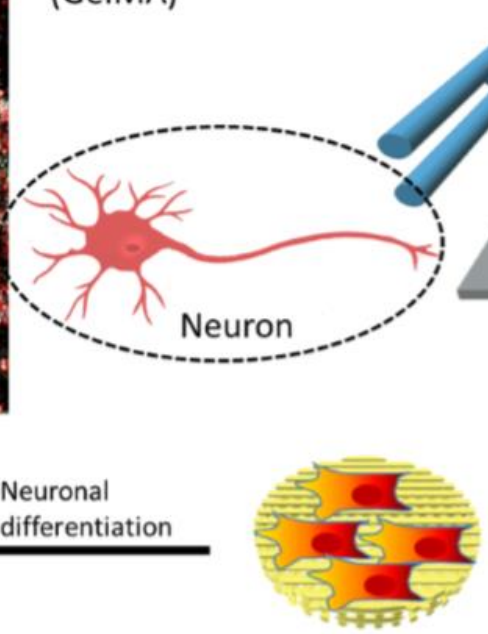

NSCs seeded on scaffold

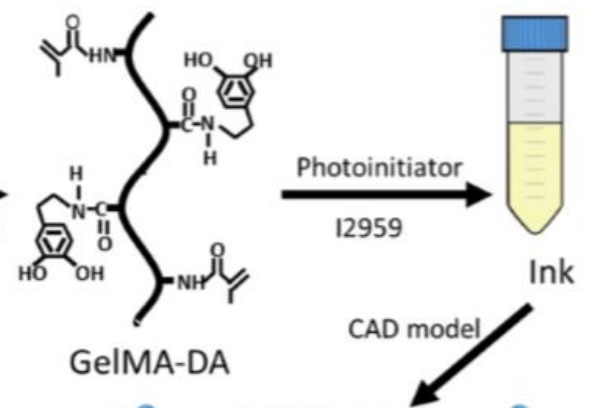

UV laser head
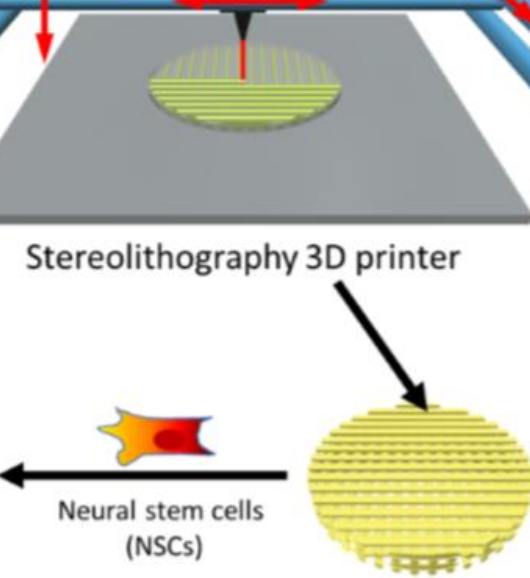

GelMA-DA scaffold

Figure 9: Schematic overview of bioink preparation and stereolithography bioprinting. Reproduced from Zhou et al. [36] with permission.

\subsubsection{Digital Light Processing}

Digital light processing (DLP) consists of a light source, a digital micromirror device (DMD), a liquid resin and a motorised stage (Figure 6B). The DMD, i.e. semiconductor array of digital light switches, controls the light intensity of each pixel. Next, the light is focussed into the liquid resin to selectively crosslink the photo-crosslinkable precursor $[165,189]$. When the desired pattern of that layer according to the CAD model is crosslinked, the motorised stage moves and the next layer is printed. Via this way, complex 3D structures can be fabricated $[165,189]$. The major benefit of DLP over conventional stereolithography is that with each flash, an entire layer is crosslinked, making it significantly faster in comparison to stereolithography [2].

It is known from various literature reports that gelatin derivatives can be successfully applied for DLP. The modified gelatins can either be used as a biomaterial ink or as bioink and are often combined with 
other materials to improve the structural fidelity of the scaffolds or to improve their mechanical properties. Grogan et al. and Gauvin et al. used gel-MA with Irgacure 2959 as a biomaterial ink for the production of micropatterned scaffolds suitable for meniscus and vascular tissue engineering respectively $[186,187]$. Both authors combined the gel-MA with $\mathrm{CaCO}_{3}$ in order to improve the structural integrity of the developed scaffolds. Using this approach, it became possible to produce micropatterned scaffolds which were able to support cellular alignment of human avascular zone meniscus cells and HUVECs. After scaffold fabrication, the $\mathrm{CaCO}_{3}$ was removed via incubation in a $\mathrm{HCl}$ solution $[186,187]$.

The nozzle-free DLP technique holds a lot of potential towards printing in the presence of cells. Soman et al. and Schuster et al. investigated the potential of a gel-MA bioink using Irgacure 2959 and Irgacure 819 as photoinitiators for various tissue engineering applications using DLP $[43,134,190]$. Soman et al. combined $10 \mathrm{w} / \mathrm{v} \%$ gel-MA with murine embryonic fibroblasts (NIH-3T3) to fabricate complex structures including spiral, pyramid, flower and dome-shaped micro-geometries [190]. The authors observed that cell encapsulation in complex 3D patterned scaffolds provided long-term control over cell viability $(>80 \%)$, cell proliferation, morphology and geometric guidance compared to conventional cell seeding methods [190]. Synthetic materials are often added to the gel-MA bioink in order to increase the mechanical properties rendering the formulation suitable for bone tissue engineering applications which often require a high mechanical strength. For example, Schuster et al. modified gel-MA with PEG derivatives (Figure 1 I) to obtain a suitable bioink for osteoblasts and endothelial cells. The results indicated that the bioink had a negligible cytotoxicity and could be processed using DLP [43].

Recently, conductive hydrogels have been developed for tissue engineering applications, because they can serve as a bioactive scaffold that can electrically stimulate cells and modulate their function. By integrating inherently conductive polymers such as polyaniline in gel-MA hydrogels, it became possible to produce electro-conductive hydrogels. Wu et al. and Sawyer et al. developed electrically conductive gel-MA-poly(aniline) (gel-MA-PANi) hydrogels which are suitable as biomaterial ink or 
bioink for DLP respectively using Irgacure 2959 as a photoinitiator [191,192]. Wu et al. showed that the swelling properties, compressive modulus, cell adhesion and spreading response of the gel-MAPANi hydrogels are similar to pure gel-MA [191]. However, the electrical properties are superior compared to gel-MA [191]. Sawyer et al. developed a gel-MA-PANi-based bioink that is suitable for the encapsulation of human osteogenic cells. The authors found out that the cell viability of the encapsulated cells within the gel-MA-PANi hydrogels was similar to pure gel-MA hydrogels. Furthermore, the cells in the gel-MA-PANi hydrogels demonstrated the capability of depositing minerals within the hydrogel matrix after being chemically induced for two weeks. Additionally, the composite hydrogel could be processed into complex shapes using DLP [192].

Miri et al. developed a DLP system which is combined with a microfluidic device containing four on/off pneumatic valves [193]. This device is capable of fast switching between four different (cell-laden) hydrogel bioinks based on PEGDA and gel-MA to achieve layer-by-layer multimaterial bioprinting. Via this way, complex 3D printed structures could be fabricated using three different bioinks including gelMA combined with osteoblasts, MSCs or fibroblasts using LAP as a biocompatible photo-initiator. In conclusion, this system provides a robust platform for on demand bioprinting of high-fidelity multimaterial microstructures for various tissue engineering, regenerative medicine and biosensing applications, which are otherwise not readily achievable at high speed with conventional stereolithographic biofabrication systems [193].

Most DLP devices operate using UV or near-UV blue light ( $405 \mathrm{~nm}$ ) which may be harmful for cells due to the long UV exposure times. Therefore, Wang et al. investigated the potential of a visible-lightcrosslinkable gelatin methacrylamide based bioink using an eosin Y PI for SLA bioprinting (Figure 10) [194]. Eosin $Y$ is a green-light sensitive photo-initiator $(500-600 \mathrm{~nm})$ which initiates a highly biocompatible crosslinking reaction [195]. Wang et al. showed that the optimal combination for SLA bioprinting was $0.02 \mathrm{mM}$ eosin $\mathrm{Y}$ with $15 \mathrm{w} / \mathrm{v} \%$ gel-MA [194]. The results indicated that the NIH-3T3 cells survived the printing process ( $>80 \%$ ) and were able to proliferate and to form 3D intercellular networks. Furthermore, Lim et al. developed a bioink based on methacrylated PVA, being a promising 
synthetic, non-toxic and hydrophilic material, gel-MA for its cell interactivity and the visible light photo-initiator system Ru/SPS using MSCs [2]. The cell-laden scaffolds developed using DLP had a complex architecture with a high resolution in which the encapsulated cells remained viable (> $85 \%)$, homogeneously distributed and functional [2].

The research of Bertlein et al. showed that also different gelatin derivatives could be processed via DLP. The authors were able to print partially hydrolysed allylated gelatin (gel-AGE) combined with DTT without the need for any photo-absorber to be present [62].

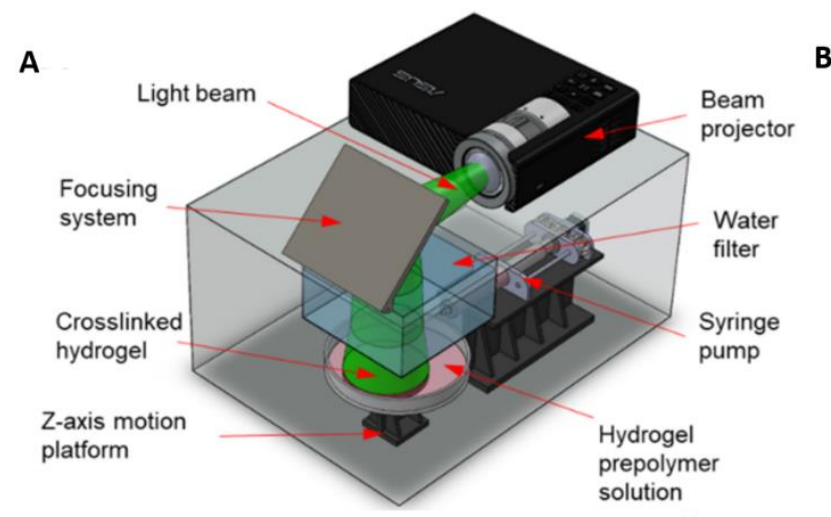

B
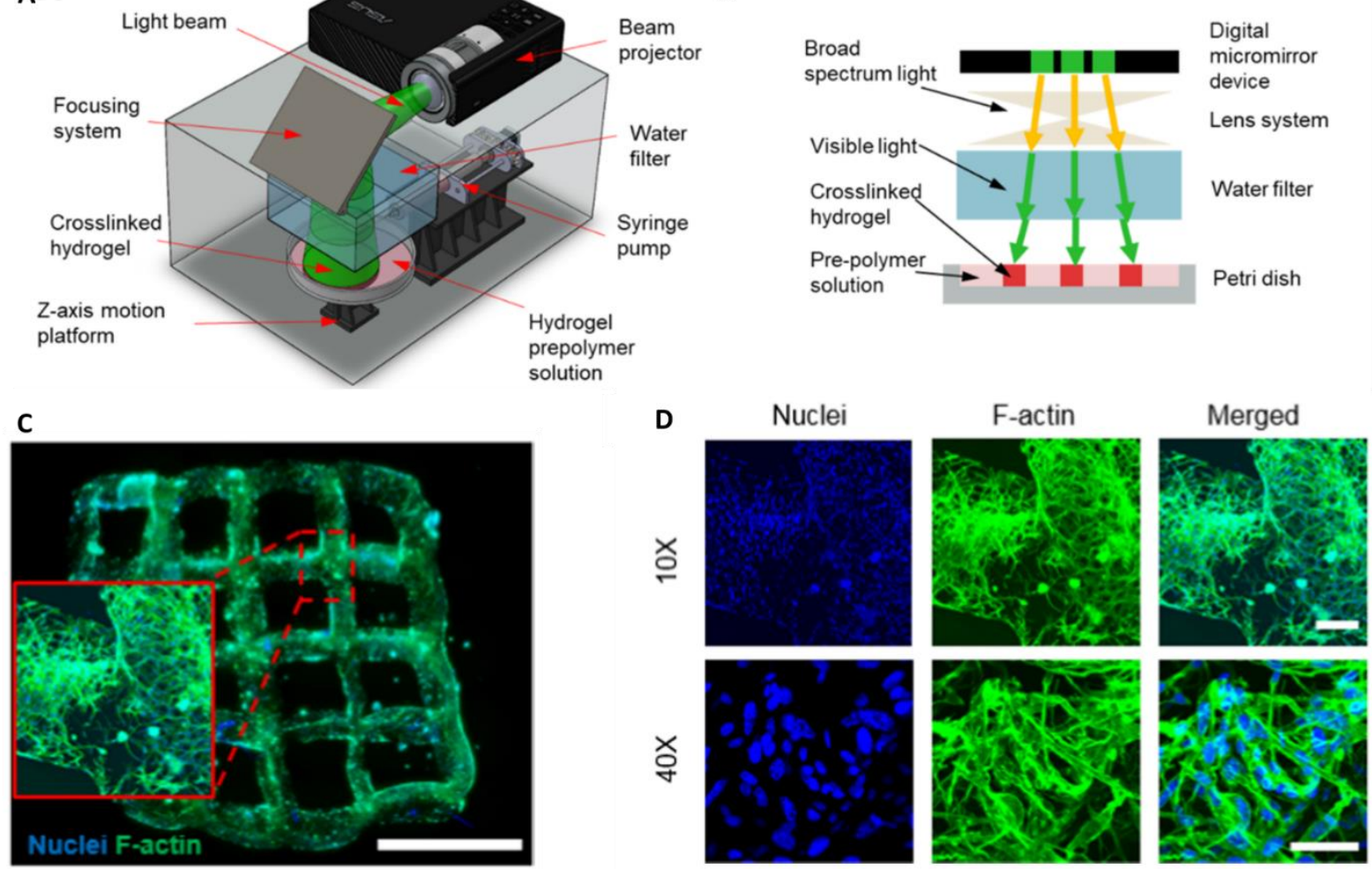

Figure 10: (A) Schematic illustration of visible-light-based DLP 3D bioprinting with the various components. (B) Schematic overview of the principles of single-layer printing. (C) NIH-3T3 cell-laden bioprinted scaffold at day 5 stained with DAPI for nuclei (blue) and phalloidin 488 for F-actin (green). The scale bar represents $2 \mathrm{~mm}$. (D) Confocal fluorescence microscopy images of a junction in the mesh pattern at 10x and 40x magnification. The scale bar represents $300 \mu \mathrm{m}(10 \mathrm{x})$ and $50 \mu \mathrm{m}(40 \mathrm{x})$. Reproduced from Wang et al. [194] with permission.

\subsubsection{Two-Photon Polymerization}

2PP-is based on the non-linear absorption of laser light to induce crosslinking in a photosensitive resin.

By tightly focusing a femtosecond laser beam into the material, the simultaneous interaction of a photo-initiator molecule with two photons, each possessing half the required energy to bridge the 
band gap required for photo-initiator excitation can be met to initiate localised free-radical polymerization [16,196,197]. Compared to conventional light-based additive manufacturing techniques using linear (i.e. single-photon) absorption, for which polymerization can occur throughout the entire beam path and is only limited by its penetration depth into the material, 2PP allows the polymerization only in a small volumetric element (voxel) enabling the fabrication of structures with resolutions below the diffraction limit. The maximum achievable resolution is determined by the size of the voxel which depends on the applied optics and laser source $[16,198,199]$. As a consequence of this unique principle, this is the only additive manufacturing technology which allows processing of gelatin in the physically crosslinked state. Moreover, processing in the physically crosslinked state not only leads to more efficient crosslinking, but also provides support to the structures during crosslinking, resulting in the possibility to generate more complicated architectures $[16,29,64]$.

In 2011, our research groups (i.e. A. Ovsianikov \& S. Van Vlierberghe) were the first to report on 2PP processing of modified gelatin (gel-MA, Figure $1 \mathrm{~A}$ ) for the generation of scaffolds for tissue engineering purposes using primary adipose tissue-derived stem cells (Figure $11 \mathrm{C}$ ) [30]. Ever since, multiple studies reported 2PP processing of modified gelatin being mainly gel-MA $[63,131,200,201]$. In 2014, Ovsianikov et al. reported the first study on 2PP in the presence of living cells [29]. Although the cells did not survive direct exposure to the laser during structuring, it was possible to use 2PP to entrap cells within 3D microstructures [29] . Furthermore, the research indicated that the cytotoxicity was not a result of the applied laser intensity, but could be attributed to the formation of cytotoxic species (i.e. singlet oxygen) within the cells as a side-product of P2CK photo-initiator activation $[29,93]$ . This hypothesis was later substantiated by the development of a macromolecular photo-initiator based on hyaluronic acid, which did enable 2PP processing combined with the encapsulation of living cells in the exposed areas as well [132]. The study indicated that the previously observed cytotoxicity originated from the penetration of the low molecular weight photo-initiator through the cell membrane, thereby resulting in photo-oxidative damage within the cell during irradiation. By immobilizing the photo-initiator onto a macromolecule, it could no longer penetrate the cell 
membrane, thereby allowing 2PP in the presence of living cells [132]. Additionally, a different approach using a type I cleavable diazosulfonate PI DAS (Figure 4) has been developed for direct encapsulation of living cells in gel-MA hydrogels. As a result, cell survival was five times higher when compared to P2CK, while maintaining high writing speeds $(1000 \mathrm{~mm} / \mathrm{s})$ thereby further demonstrating its potential as a biocompatible photo-initiator for 2PP [127] (Figure $11 \mathrm{E}$ ).

Despite these successful approaches, gelatin-methacryloyl is characterized by some limitations in the context of 2PP processing. In general, the poor reaction kinetics and associated mechanical properties require relatively high light doses (e.g. $70 \mathrm{~mW}$ at $1000 \mathrm{~mm} / \mathrm{s}$ scan speed) to crosslink the material. Furthermore, the subsequent swelling of the 2PP-produced structures can compromise the highresolution capacity of this technology [127].

There are several approaches which have already been developed to overcome the poor mechanical properties and low reactivity associated with gel-MA for 2PP structuring. The mechanical properties could be improved by using a secondary material to function as/contribute to mechanical support $[50,130,201]$. A second strategy consisted of co-crosslinking low concentrations of PEGDA (1\%) for the formation of a co-network. In this respect, processing benefits from the higher mechanical properties of PEG, along with superior acrylate-based reaction kinetics [130]. Alternatively, benefitting from an indirect approach, first a stronger material (e.g. a mixture of hydrophobic acrylates) can be structured to function as support, followed by subsequent gel-MA structuring [201] .

Another approach to improve the properties of gel-MA is to modify the material chemically. To this end, Van Hoorick et al. developed a gelatin derivative of which all primary amines were modified into methacrylamides $(0.385 \mathrm{mmol} / \mathrm{g}$ gelatin), while additional methacrylates were introduced onto the carboxylic acids, resulting in $1 \mathrm{mmol}$ crosslinkable groups per gram gelatin (Figure $1 \mathrm{~B}$ ) [16,76]. As a consequence, a denser gelatin network can be formed exhibiting both higher stiffness along with less to no occurrence of post-production swelling. Additionally, the reaction kinetics were improved compared to conventional gelatin-methacrylamide thereby resulting in a broader 2PP spatiotemporal processing range (Figure 11 A) $[16,76]$. Furthermore, 2D biocompatibility experiments indicated a 
comparable biocompatibility towards both fibroblasts (L929) and osteoblasts (MC3T3) for gel-MODAEMA and the well-established gel-MA [16] .

Although the introduction of these additional functionalities resulted in a drastic improvement in terms of 2PP processing, the crosslinking reactions remain subject to the drawbacks associated with chain-growth polymerizable hydrogels as discussed earlier. Therefore, to further improve the material processing range, 2PP experiments have also been explored using thiol-ene photoclick hydrogels [63]. Qin et al. reported the synthesis of gelatin hydrolysate vinyl esters which were copolymerized with reduced derivatives of bovine serum albumin as a thiolated crosslinker. In a different system, gelatin type B was modified with norbornene functionalities (Figure $1 \mathrm{k}$ ) [63]. Gel-NB was processed via 2PP using DTT as thiolated crosslinker resulting in a drastically improved spatiotemporal 2PP processing range compared to all previously reported gelatin derivatives. On the one hand, only half of the energy was required to result in reproducible crosslinking (i.e. $20 \mathrm{~mW}$ at $100 \mathrm{~mm} / \mathrm{s}$ for gel-NB + DTT DS 63 vs $40 \mathrm{~mW}$ at $100 \mathrm{~mm} / \mathrm{s}$ for gel-MOD-AEMA) despite a four times decreased concentration of crosslinkable functionalities (i.e. $0.24 \mathrm{mmol} / \mathrm{g}$ for gel-NB vs $1 \mathrm{mmol} / \mathrm{g}$ for gel-MOD-AEMA). Additionally, from $40 \mathrm{~mW}$ onwards, further increasing the laser power did not influence the hydrogel swelling behaviour, which indicated that the material was already fully crosslinked, in contrast to gelMOD-AEMA for which a further increase of the laser power resulted in concomitantly decreasing swelling ratios $[16,64]$. Furthermore, also a broader concentration range could be applied for 2PP processing, since reproducible structuring was reported for the first time below a $10 \mathrm{w} / \mathrm{v} \%$ gelatin concentration (i.e. $5 \mathrm{w} / \mathrm{v} \%$ ) [64]. It should be noted that when comparing to gel-MA with a comparable DS, gel-NB is characterized by significantly lower swelling ratios due to the higher degree of conversion during structuring [64]. As a consequence, a superior CAD-CAM mimicry is observed when using gelNB + DTT in comparison to gel-MA, while the lower required spatiotemporal energy for full conversion leads to stiffer gels at lower laser powers. As a consequence, the material could also be applied for the fabrication of complex structures able to support their own weight despite the presence of only 
small support structures or micro-scaffolds, which were fully populated by fibroblasts after 7 days of cell culture (Figure 11 A) [64] .

Another application of 2PP-assisted photomanipulation of gelatin-based hydrogels has been reported by Pennacchio et al. They incorporated an azobenzene crosslinker into acrylamide-modified gelatin

(Figure $1 \mathrm{~F}$, Figure $11 \mathrm{D}$ ) hydrogels. Upon 2PP illumination, the azobenzene molecules undergo isomerization from the more planar (trans) to a bent (cis) conformation. This transformation triggers changes in the material properties such as the mesh size, stiffness and/or its swelling behaviour resulting in a dynamic hydrogel platform for 3D cell culture (Figure 11 D) [39].

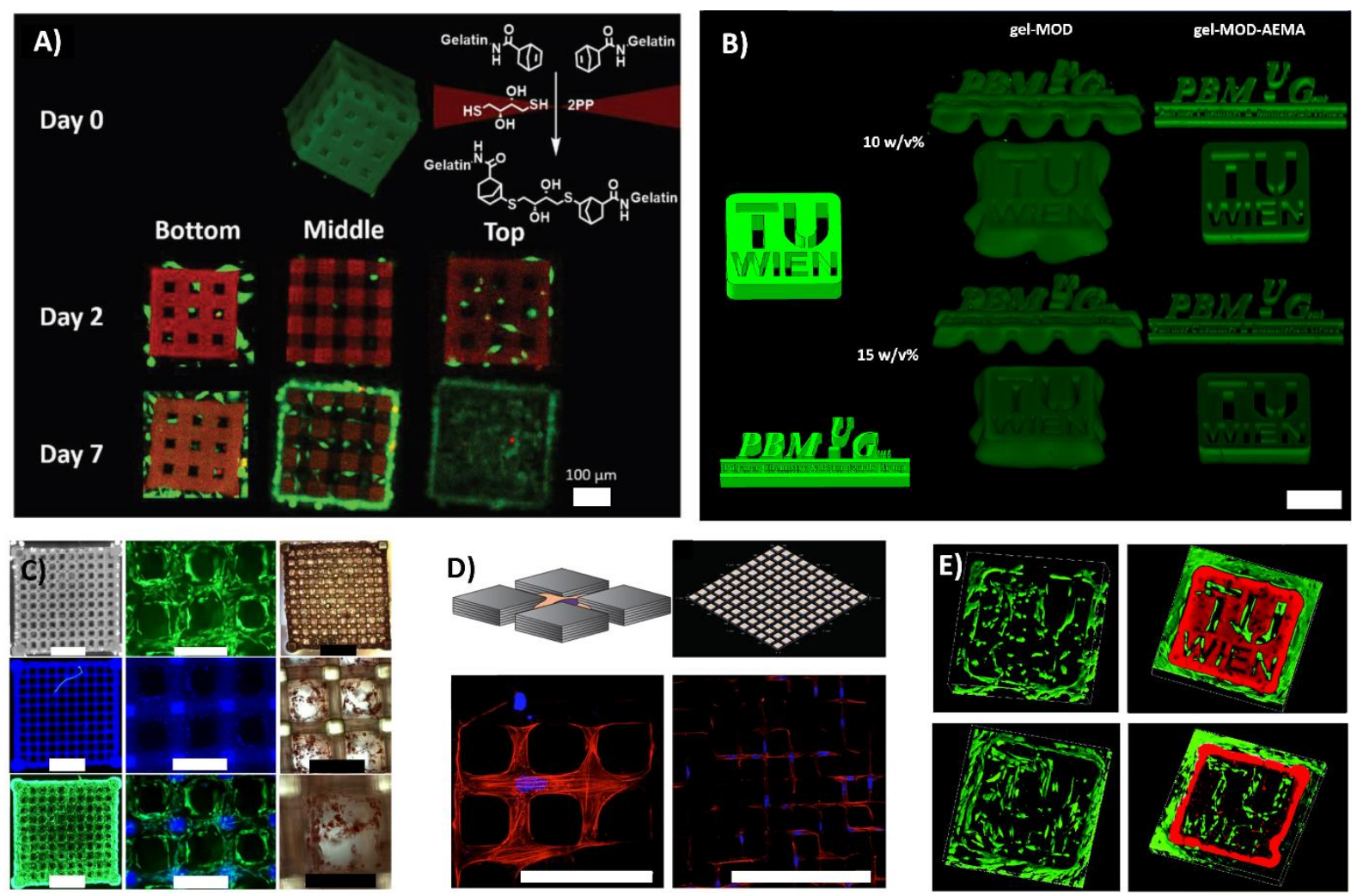

Figure 11: (A.) Scheme demonstrating the thiol-ene photoclick crosslinking of gelatin into a microscaffold, subsequent cell culture in the presence of $L 929$ fibroblasts after 2 and 7 days cell culture (reproduced from [64] with permission). The scale bar represents $100 \mu \mathrm{m}$. (B.) Difference in shape fidelity between gel-MOD and gel-MOD-AEMA due to post-production swelling as compared to the CAD model. (scale bars represent $100 \mu \mathrm{m}$ ) (Image adapted from [16] with permission; copyright 2017 ACS (https://pubs.acs.org/doi/abs/10.1021\%2Facs.biomac.7b00905)). (C) First reported gelatin scaffold obtained via 2PP seeded with primary adipose-derived stem cells. The scale bars represent from top to bottom $1000 \mu \mathrm{m}, 300 \mu \mathrm{m}$ and $200 \mu \mathrm{m}$ respectively) (Reprinted with permission from [30] under the CC BY 3.0) (D) Micropattern of a photoresponsive gelatin derivative, enabling light-based control over swelling properties (reprinted with permission from [39]). The scale bar represents 100 $\mu \mathrm{m}(\mathrm{E})$ 2PP structures recorded in gel-MA hydrogels, using DAS (left) and P2CK (right) as PI, thereby proving viability of the cells (green cells) inside the structured material when using DAS. The red signal 
shown for the P2CK samples is caused by the autofluorescence of the $2 \mathrm{PI}$. The dimensions of the structures are $500 \times 500 \times 125 \mu \mathrm{m}^{3}$ (reprinted with permission from [127]).

\section{Processability of gelatin derivatives using additive manufacturing technologies.}

Table 1 provides a non-exhaustive overview of the processability of all reported gelatin derivatives using additive manufacturing technologies. Furthermore, if a specific derivative hasn't been reported for a certain processing technology to date, a reasonable estimation of processability using that particular technology is presented based on the properties of the derivative. The symbol ' $\checkmark$ ' is applied if no difficulties towards processing are anticipated based on previous experiments with similar derivatives, ' $x$ ' represents improbable processability while ' $\checkmark / x^{\prime}$ refers to the fact that processability is anticipated upon thorough adaptation of the printing technology (i.e. heating of the resin bath for SLA/DLP, or in situ UV crosslinking during deposition when using syringe based printers) and/or severe optimisation of the printing parameters (e.g. slower printing speed).

The hypotheses were based on the following criteria:

For inkjet processing, it can be anticipated that the derivative will only exhibit the correct viscosity range if the modification induces solubility at room temperature as reported by Hoch et al. and discussed in section 4.2 [37]. Furthermore, syringe-based processing is likely to be possible if the material exhibits physical gelation at room temperature while enabling either subsequent crosslinking or crosslinking during deposition. For example, when a multicomponent system is used for which spontaneous crosslinking occurs upon mixing, this can be accomplished either by using a mixing needle or if one component is printed in a container containing the other material.

For the light-based processes (i.e. SLA, DLP, 2PP), processability is anticipated if photo-crosslinking occurs within a reasonable time frame (i.e. seconds to minutes depending on the applied technique). As a second requirement, for SLA and DLP, the derivatives should still be soluble at room temperature as discussed in section 4.2. If the material forms a gel, it is denoted with ' $\checkmark / x$ to indicate that the material is likely to be processable if the process occurs at elevated temperatures thereby inducing gel to sol transition. Finally, for 2PP processing, the material can be crosslinked both in liquid and in gel state, and therefore a derivative is considered processable if the material is photo-crosslinkable 
within a reasonable time frame(i.e. seconds to minutes) and if there is a possibility to add a 2PP-active photoinitiator to the formulation.

Table 1: Non-exhaustive overview of additive manufacturing processability of the gelatin derivatives discussed in Figure 1 based on crosslinking mechanism. In colour, the references in which the specific printing technology was reported are presented. In grey, the anticipated processability using the respective technologies is depicted.

\begin{tabular}{|c|c|c|c|c|c|c|c|c|c|c|c|}
\hline Derivative & $\begin{array}{l}\text { Figure } 1 \\
\text { ref. }\end{array}$ & Ink jet & Syringe & SLA & DLP & 2PP & $\begin{array}{l}\text { Ink } \\
\text { jet }\end{array}$ & $\begin{array}{l}\text { Syrin } \\
\text { ge }\end{array}$ & SLA & DLP & 2PP \\
\hline \multicolumn{12}{|c|}{ Chain Growth Polymerization Derivatives } \\
\hline gel-MA & A & [37][202] & {$[27][203][204][205]$} & {$[206]$} & {$[207][208]$} & $\begin{array}{l}{[127][29]} \\
{[132][30]} \\
{[200]}\end{array}$ & & & & & \\
\hline gel-MOD-AEMA & B & & & & & {$[64][76]$} & $\checkmark$ & $\checkmark / x$ & $\checkmark$ & $\checkmark$ & \\
\hline gel-MA-DA & C & & & {$[36]$} & & & $\checkmark$ & $\sqrt{ } / x$ & & $\checkmark$ & $\checkmark$ \\
\hline GMA & $\mathrm{D}$ & [37] & [209] & & & & & & $\checkmark$ & $\checkmark$ & $\checkmark$ \\
\hline gel-AA & $E$ & & & & & & $x$ & $\checkmark$ & $\sqrt{ } / x$ & $\checkmark / x$ & $\checkmark$ \\
\hline $\begin{array}{l}\text { gelatin- } \\
\text { acrylamide }\end{array}$ & $\mathrm{F}$ & & & & & [39] & $x$ & $\checkmark$ & $\checkmark / x$ & $\sqrt{ } / x$ & \\
\hline gel-BTHE & G & & & & & & $\sqrt{ } / x$ & $\checkmark$ & $\checkmark / x$ & $\checkmark / x$ & $\checkmark / x^{a}$ \\
\hline gel-Boc-AEMA & $\mathrm{H}$ & & & & & & $\checkmark$ & $\checkmark / x$ & $\checkmark$ & $\checkmark$ & $\checkmark$ \\
\hline MPG & I & & & [43] & {$[42]$} & & $\checkmark$ & $\sqrt{ } / x$ & & & $\checkmark$ \\
\hline gelatin-PEG & $\mathrm{K}$ & & & & & & $x$ & $\checkmark$ & $\checkmark / x$ & $\checkmark / x$ & $\checkmark$ \\
\hline \multicolumn{12}{|c|}{ Thiolated Gelatins } \\
\hline gel-SH & $\mathrm{J}$ & & & & & & $x$ & $\checkmark$ & $\sqrt{ } / x^{b}$ & $\sqrt{ } / x^{b}$ & $\sqrt{b}$ \\
\hline gel-SH & $\mathrm{L}$ & & {$[46]^{\mathrm{b}}$} & & & & $x$ & $\checkmark$ & $\sqrt{ } / x^{b}$ & $\sqrt{ } / x^{b}$ & $\sqrt{\mathrm{b}}$ \\
\hline $\begin{array}{l}\text { Aminated- } \\
\text { thiolated-gelatin }\end{array}$ & $\mathrm{N}$ & & & & & & $\sqrt{ } / x$ & $\sqrt{ } / x$ & $\sqrt{ } / x^{b}$ & $\sqrt{ } / x^{b}$ & $\sqrt{b}$ \\
\hline $\begin{array}{l}\text { gelatin-Cys-2- } \\
\text { MPD }\end{array}$ & 0 & & & & & & $x$ & $\checkmark$ & $x$ & $x$ & $x$ \\
\hline gelatin-Cys & $P$ & & & & & & $x$ & $\checkmark$ & $\sqrt{ } / x^{b}$ & $\sqrt{ } / x^{b}$ & $\sqrt{\mathrm{b}}$ \\
\hline gel-PEG-Cys & $Q$ & & & & & & $x$ & $\checkmark$ & $\sqrt{ } / x^{b}$ & $\sqrt{ } / x^{b}$ & $\sqrt{\mathrm{b}}$ \\
\hline gelatin-TBA-MNA & $\mathrm{R}$ & & & & & & $x$ & $\checkmark$ & $x$ & $x$ & $x$ \\
\hline gel-S & $S$ & & & & & & $x$ & $\checkmark$ & $\sqrt{ } / x^{b}$ & $\checkmark / x^{b}$ & $\sqrt{\mathrm{b}}$ \\
\hline $\begin{array}{l}\text { gelatin- } \\
\text { thiobutyrolacton }\end{array}$ & $T$ & & & & & & $x$ & $\checkmark$ & $\sqrt{ } / x^{b}$ & $\sqrt{ } / x^{b}$ & $\sqrt{b}$ \\
\hline \multicolumn{12}{|c|}{ Derivatives for Enzymatic Crosslinking } \\
\hline gelatin-tyramine & $U$ & {$[210][211][212]$} & {$[124]$} & & & & & & $\sqrt{ } / x$ & $\sqrt{ } / x$ & $\checkmark$ \\
\hline $\begin{array}{l}\text { gelatin/tyramine/ } \\
\text { heparin }\end{array}$ & $\mathrm{V}$ & & & & & & $\checkmark / x$ & $\checkmark$ & $\checkmark$ & $\checkmark$ & $\checkmark$ \\
\hline \multicolumn{12}{|c|}{ Derivatives for Photo-Oxidation } \\
\hline gelatin-FA & W & & & & & & $x$ & $\checkmark$ & $\sqrt{ } / x$ & $\sqrt{ } / x$ & $\checkmark$ \\
\hline gelatin-FI & $\mathrm{X}$ & & & & & & $x$ & $\checkmark$ & $\sqrt{ } / x$ & $\sqrt{ } / x$ & $\checkmark$ \\
\hline gel-FGE & $\mathrm{Y}$ & & {$[56][57]$} & & & & $x$ & & $\checkmark / x$ & $\checkmark / x$ & $\checkmark$ \\
\hline \multicolumn{12}{|c|}{ Derivatives for $\pi-\pi$ Cycloaddition } \\
\hline gel-MFVF & Z & & & & & & $x$ & $\checkmark / x$ & $\sqrt{ } / x$ & $\checkmark / x$ & $\checkmark / x$ \\
\hline gel-AC & $\alpha$ & & & & & & $x$ & $x$ & $x$ & $x$ & $x$ \\
\hline gel-NC & $\beta$ & & & & & & $x$ & $\checkmark$ & $\sqrt{ } / x$ & $\checkmark / x$ & $\sqrt{ } / x$ \\
\hline \multicolumn{12}{|c|}{ Derivatives for Diels-Alder Click Chemistry } \\
\hline gel-furan & $Y$ & & & & & & $x$ & $\checkmark / x$ & $\sqrt{ } / x^{c}$ & $\checkmark / x^{c}$ & $\sqrt{c}$ \\
\hline gel-FGE & $\delta$ & & & & & & $x$ & $\sqrt{ } / x$ & $\sqrt{ } / x^{c}$ & $\sqrt{ } / x^{c}$ & $\sqrt{ } \mathrm{c}$ \\
\hline gel-NB & $\varepsilon$ & & & & & & $\checkmark / x^{e}$ & $\checkmark$ & $\sqrt{ } / x^{d}$ & $\checkmark / x^{d}$ & $\checkmark d$ \\
\hline gel-T & $\zeta$ & & & & & & $\sqrt{ } / x^{\mathrm{e}}$ & $\checkmark$ & $x$ & $x$ & $x$ \\
\hline \multicolumn{12}{|c|}{ "ene" Derivatives for Thiol-ene Chemistry } \\
\hline $\begin{array}{l}\text { gelatin- } \\
\text { pentenoate }\end{array}$ & $\eta$ & & & & & & $x$ & $\checkmark^{d}$ & $\sqrt{ } / x^{d}$ & $\sqrt{ } / x^{d}$ & $\checkmark^{d}$ \\
\hline gel-AGE & $\theta$ & & {$[62]^{d}$} & & {$[62]^{d}$} & & $x$ & & $\sqrt{d}$ & & $\sqrt{d}$ \\
\hline gel-VE & $\mathrm{L}$ & & & & & {$[63]^{d}$} & $x$ & $\checkmark d$ & $\sqrt{ } / x^{d}$ & $\checkmark / x^{d}$ & \\
\hline gel-NB & $\mathrm{K}$ & & {$[46]^{d}$} & & & {$[64]^{d}$} & $x$ & & $\sqrt{ } / x^{d}$ & $\checkmark / x^{d}$ & \\
\hline gel-NB & $\lambda$ & & & & & & $x$ & $\sqrt{d}$ & $\sqrt{ } / x^{d}$ & $\sqrt{ } / x^{d}$ & $\sqrt{d}$ \\
\hline gel-NB & $\mu$ & & & & & & $x$ & $\checkmark d$ & $\sqrt{ } / x^{d}$ & $\sqrt{ } / x^{d}$ & $\sqrt{d}$ \\
\hline
\end{tabular}

alt is anticipated that 2PP the coupled benzophenone moiety is 2 photon active as there are numerous reported benzophenone based $2 \mathrm{PP}$ Photoinitiators[213][214][215].

b In the presence of an ene-containing crosslinker. 
'Using a photo-oxidation approach

dIn the presence of thiolated crosslinker

eWhen using a drop on drop method of both components if the correct viscosity is obtained

\section{Conclusions}

Throughout the past two decades, a plethora of photo-crosslinkable gelatins suitable for tissue engineering purposes have emerged. Although a large number of crosslinkable chemistries, each characterized by their specific benefits and drawbacks, have been reported, the majority of the reported gelatin derivatives apply a chain growth crosslinking system (e.g. gel-MA). However, a second important chemistry which is gaining increasing attention in the field is thiol-ene (photo-)click chemistry, which exhibits substantial benefits for light-based biofabrication strategies due to an increased reactivity and material tunability. Besides the most important crosslinking chemistries, a lot of alternatives have also been investigated. Unfortunately, most of these alternatives still remain in the proof of concept stage without actual applications. However, the reported successful biofabrication strategies of the more conventional derivatives in combination with a desirable biocompatibility, cell interactivity and cost-effectiveness, have resulted in the commercialisation of the most common crosslinkable gelatin derivative (gel-MA) for research purposes. Due to the recent successes accomplished with thiol-ene based systems for biofabrication purposes, it is anticipated that thiol-ene derivatives will also penetrate into the research market. It is anticipated that off-the-shelf availability of these materials can drastically decrease the applied research learning curve. This, in combination with gelatin's wide applicability and the declining cost trend characteristic for additive manufacturing technologies, can likely induce a paradigm shift towards high-end biofabrication breakthroughs along with their integration into a clinical setting. Since gelatin is already FDA-approved with widespread applications in the food and pharmaceutical industry, it is only a matter of time until biofabrication strategies using photo-crosslinkable gelatins will be conventional in the clinic. 


\section{Acknowledgement}

Jasper Van Hoorick and Liesbeth Tytgat were granted an FWO-SB PhD grant provided by the Research Foundation Flanders (FWO, Belgium). The FWO-FWF grant (a bilateral Research foundation Flanders Austrian Science fund project) is acknowledged for financial support. S. Van Vlierberghe would like to thank the FWO for financial support under the form of research grants (G005616N, G0F0516N, FWOKN273, G044516N) as well as Ghent University for funding a starting grant through the Special Research Fund.

For permissions related to using the material of Figure $2 \mathrm{D}$ and Figure $11 \mathrm{~B}$ ACS should be contacted.

\section{References}

[1] J. Groll, J.A. Burdick, D. Cho, B. Derby, M. Gelinsky, S.C. Heilshorn, T. Jüngst, J. Malda, A definition of bioinks and their distinction from biomaterial inks, Biofabriation. 11 (2019) 1-5.

[2] K.S. Lim, R. Levato, P.F. Costa, M.D. Castilho, C.R. Alcala-Orozco, K.M.A. Van Dorenmalen, F.P.W. Melchels, D. Gawlitta, G.J. Hooper, J. Malda, T.B.F. Woodfield, Bio-resin for high resolution lithography-based biofabrication of complex cell-laden constructs, Biofabrication. 10 (2018). doi:10.1088/1758-5090/aac00c.

[3] L. Moroni, T. Boland, J.A. Burdick, C. De Maria, B. Derby, G. Forgacs, J. Groll, Q. Li, J. Malda, V.A. Mironov, C. Mota, M. Nakamura, W. Shu, S. Takeuchi, T.B.F. Woodfield, T. Xu, J.J. Yoo, G. Vozzi, Biofabrication: A Guide to Technology and Terminology, Trends Biotechnol. 36 (2018) 384-402. doi:10.1016/j.tibtech.2017.10.015.

[4] Z. Li, T. Qu, C. Ding, C. Ma, H. Sun, S. Li, X. Liu, Injectable gelatin derivative hydrogels with sustained vascular endothelial growth factor release for induced angiogenesis, Acta Biomater. 13 (2015) 88-100. doi:10.1016/j.actbio.2014.11.002.

[5] J.F. Pan, H.F. Yuan, C.A. Guo, J. Liu, X.H. Geng, T. Fei, S. Li, W.S. Fan, X.M. Mo, Z.Q. Yan, Onestep cross-linked injectable hydrogels with tunable properties for space-filling scaffolds in tissue engineering, RSC Adv. 5 (2015) 40820-40830. doi:10.1039/c5ra02588e.

[6] I. Steyaert, H. Rahier, S. Van Vlierberghe, J. Olijve, K. De Clerck, Gelatin nanofibers: Analysis of 
triple helix dissociation temperature and cold-water-solubility, Food Hydrocoll. 57 (2016) 200208. doi:10.1016/j.foodhyd.2016.01.016.

[7] R. Schrieber, H. Gareis, Gelatine Handbook: Theory and Industrial Practice, 2007.

[8] J. Van Hoorick, H. Declercq, A. De Muynck, A. Houben, L. Van Hoorebeke, R. Cornelissen, J. Van Erps, H. Thienpont, P. Dubruel, S. Van Vlierberghe, Indirect additive manufacturing as an elegant tool for the production of self-supporting low density gelatin scaffolds., J. Mater. Sci. Mater. Med. 26 (2015) 247. doi:10.1007/s10856-015-5566-4.

[9] a I. Van Den Bulcke, B. Bogdanov, N. De Rooze, E.H. Schacht, M. Cornelissen, H. Berghmans, Structural and rheological properties of methacrylamide modified gelatin hydrogels., Biomacromolecules. 1 (2000) 31-38. doi:10.1021/bm990017d.

[10] J. Van Hoorick, P. Gruber, M. Markovic, M. Tromayer, J. Van Erps, H. Thienpont, R. Liska, A. Ovsianikov, P. Dubruel, S. Van Vlierberghe, Crosslinkable Gelatins with Superior Mechanical Properties Through Carboxylic Acid Modification : Increasing the Two-Photon Polymerization $\begin{array}{llll}\text { Potential. } & \text { (Supporting Information), }\end{array}$ doi:10.1021/acs.biomac.7b00905.

[11] S. Sakai, K. Hirose, K. Taguchi, Y. Ogushi, K. Kawakami, An injectable, in situ enzymatically gellable, gelatin derivative for drug delivery and tissue engineering, Biomaterials. 30 (2009) 3371-3377. doi:10.1016/j.biomaterials.2009.03.030.

[12] I. Van Nieuwenhove, A. Salamon, K. Peters, G. Graulus, J.C. Martins, D. Frankel, K. Kersemans, F. De Vos, S. Van Vlierberghe, P. Dubruel, Gelatin- and starch-based hydrogels. Part A : Hydrogel development, characterization and coating, Carbohydr. Polym. 152 (2016) 129-139. doi:10.1016/j.carbpol.2016.06.098.

[13] G.-J. Graulus, A. Mignon, S. Van Vlierberghe, H. Declercq, K. Fehér, M. Cornelissen, J.C. Martins, P. Dubruel, Cross-linkable alginate-graft-gelatin copolymers for tissue engineering applications, Eur. Polym. J. (2015). doi:10.1016/j.eurpolymj.2015.06.033.

[14] T. Greene, C. Lin, Modular Cross-Linking of Gelatin-Based Thiol - Norbornene Hydrogels for in 
Vitro 3D Culture of Hepatocellular Carcinoma Cells, ACS Biomater. Sci. Eng. 1 (2015) 13141323. doi:10.1021/acsbiomaterials.5b00436.

[15] S.T. Koshy, R.M. Desai, P. Joly, J. Li, R.K. Bagrodia, S.A. Lewin, N.S. Joshi, D.J. Mooney, ClickCrosslinked Injectable Gelatin Hydrogels, Adv. Healthc. Mater. 5 (2016) 541-547. doi:10.1002/adhm.201500757.

[16] J. Van Hoorick, P. Gruber, M. Markovic, M. Tromayer, J. Van Erps, H. Thienpont, R. Liska, A. Ovsianikov, P. Dubruel, S. Van Vlierberghe, Cross-Linkable Gelatins with Superior Mechanical Properties Through Carboxylic Acid Modification: Increasing the Two-Photon Polymerization Potential, Biomacromolecules. (2017). doi:10.1021/acs.biomac.7b00905.

[17] T. Greene, T. Lin, O.M. Andrisani, C. Lin, Comparative study of visible light polymerized gelatin hydrogels for 3D culture of hepatic progenitor cells, J. Appl. Polym. Sci. 44585 (2017) 1-10. doi:10.1002/app.44585.

[18] A. Ito, A. Mase, Y. Takizawa, M. Shinkai, H. Honda, K.-I. Hata, M. Ueda, T. Kobayashi ' ", Transglutaminase-Mediated Gelatin Matrices Incorporating Cell Adhesion Factors as a Biomaterial for Tissue Engineering, J. Biosci. Bioeng. 95 (2003) 196-199. doi:10.1263/jbb.95.196.

[19] Gelatin, Report No. 58, FDA's SCOGS Database, Rep. No. 58, ID Code 9000-70-8. (1979).

[20] J.B. Rose, S. Pacelli, A.J. El Haj, H.S. Dua, A. Hopkinson, L.J. White, F.R. a J. Rose, Gelatin-based materials in ocular tissue engineering, Materials (Basel). 7 (2014) 3106-3135. doi:10.3390/ma7043106.

[21] S. Van Vlierberghe, Crosslinking strategies for porous gelatin scaffolds, J. Mater. Sci. 51 (2016) 1-9. doi:10.1007/s10853-016-9747-4.

[22] a. Bigi, G. Cojazzi, S. Panzavolta, K. Rubini, N. Roveri, Mechanical and thermal properties of gelatin films at different degrees of glutaraldehyde crosslinking, Biomaterials. 22 (2001) 763768. doi:10.1016/S0142-9612(00)00236-2.

[23] E. Schacht, A.I. Van Den Bulcke, B. Delaey, J.-P. Draye, Medicaments based on polymers 
composed of methacrylamide-modified gelatin, 2002.

[24] H. Shih, T. Greene, M. Korc, C. Lin, Modular and Adaptable Tumor Niche Prepared from Visible Light Initiated Thiol-Norbornene Photopolymerization, Biomacromolecules. 17 (2016) 38723882. doi:10.1021/acs.biomac.6b00931.

[25] L. Brigo, A. Urciuolo, S. Giulitti, G. Della, M. Tromayer, R. Liska, N. Elvassore, G. Brusatin, 3D high-resolution two-photon crosslinked hydrogel structures for biological studies, Acta Biomater. 55 (2017) 373-384. doi:10.1016/j.actbio.2017.03.036.

[26] B.J. Klotz, D. Gawlitta, A.J.W.P. Rosenberg, J. Malda, F.P.W. Melchels, Gelatin-Methacryloyl Hydrogels: Towards Biofabrication-Based Tissue Repair, Trends Biotechnol. 34 (2016) 394-407. doi:10.1016/j.tibtech.2016.01.002.

[27] T. Billiet, E. Gevaert, T. De Schryver, M. Cornelissen, P. Dubruel, The 3D printing of gelatin methacrylamide cell-laden tissue-engineered constructs with high cell viability., Biomaterials. 35 (2014) 49-62. doi:10.1016/j.biomaterials.2013.09.078.

[28] M. Markovic, J. Van Hoorick, K. Hölzl, M. Tromayer, P. Gruber, S. Nürnberger, P. Dubruel, S. Van Vlierberghe, R. Liska, A. Ovsianikov, Hybrid Tissue Engineering Scaffolds by Combination of Three-Dimensional Printing and Cell Photoencapsulation, J. Nanotechnol. Eng. Med. 6 (2015) 021004. doi:10.1115/1.4031466.

[29] A. Ovsianikov, S. Mühleder, J. Torgersen, Z. Li, X.-H. Qin, S. Van Vlierberghe, P. Dubruel, W. Holnthoner, H. Redl, R. Liska, J. Stampfl, Laser photofabrication of cell-containing hydrogel constructs., Langmuir. 30 (2014) 3787-94. doi:10.1021/la402346z.

[30] A. Ovsianikov, A. Deiwick, S. Van Vlierberghe, M. Pflaum, M. Wilhelmi, P. Dubruel, B. Chichkov, Laser Fabrication of 3D Gelatin Scaffolds for the Generation of Bioartificial Tissues, Materials (Basel). 4 (2011) 288-299. doi:10.3390/ma4010288.

[31] J. Van Rie, H. Declercq, J. Van Hoorick, M. Dierick, L. Van Hoorebeke, R. Cornelissen, H. Thienpont, P. Dubruel, S. Van Vlierberghe, Cryogel-PCL combination scaffolds for bone tissue repair., J. Mater. Sci. Mater. Med. 26 (2015) 5465. doi:10.1007/s10856-015-5465-8. 
[32] D. Loessner, C. Meinert, E. Kaemmerer, L.C. Martine, K. Yue, P.A. Levett, T.J. Klein, F.P.W. Melchels, A. Khademhosseini, D.W. Hutmacher, Functionalization, preparation and use of cellladen gelatin methacryloyl - based hydrogels as modular tissue culture platforms, Nat. Protoc. 11 (2016) 727-746. doi:10.1038/nprot.2016.037.

[33] K. Yue, G. Trujillo-de Santiago, M.M. Alvarez, A. Tamayol, N. Annabi, A. Khademhosseini, Synthesis, properties, and biomedical applications of gelatin methacryloyl (GelMA) hydrogels, Biomaterials. 73 (2015) 254-271. doi:10.1016/j.biomaterials.2015.08.045.

[34] K. Hölzl, S. Lin, L. Tytgat, S. Van Vlierberghe, L. Gu, A. Ovsianikov, Bioink properties before, during and after 3D bioprinting, Biofabrication. 8 (2016) 032002. doi:10.1088/1758$5090 / 8 / 3 / 032002$.

[35] Allevi3D, (n.d.).

[36] X. Zhou, H. Cui, M. Nowicki, S. Miao, S.J. Lee, F. Masood, B.T. Harris, L.G. Zhang, ThreeDimensional-Bioprinted Dopamine-Based Matrix for Promoting Neural Regeneration, ACS Appl. Mater. Interfaces. 10 (2018) 8993-9001. doi:10.1021/acsami.7b18197.

[37] E. Hoch, T. Hirth, G.E.M. Tovar, K. Borchers, Chemical tailoring of gelatin to adjust its chemical and physical properties for functional bioprinting, J. Mater. Chem. B. 1 (2013) 5675-5685. doi:10.1039/c3tb20745e.

[38] T. Billiet, B. Van Gasse, E. Gevaert, M. Cornelissen, J.C. Martins, P. Dubruel, Quantitative Contrasts in the Photopolymerization of Acrylamide and Methacrylamide-Functionalized Gelatin Hydrogel Building Blocks, Macromol. Biosci. 13 (2013) 1531-1545. doi:10.1002/mabi.201300143.

[39] F.A. Pennacchio, C. Fedele, S. De Martino, S. Cavalli, R. Vecchione, P.A. Netti, ThreeDimensional Microstructured Azobenzene-Containing Gelatin as a Photoactuable Cell Confining System, ACS Appl. Mater. Interfaces. 10 (2018) 91-97. doi:10.1021/acsami.7b13176.

[40] F.-C. Ding, S. Hsu, W.-Y. Chiang, Synthesis of a new photoreactive gelatin with BTDA and HEMA derivatives, J. Appl. Polym. Sci. 109 (2008) 589-596. doi:10.1002/app. 
[41] I. Van Nieuwenhove, S. Birgit, G.-J. Graulus, S. Van Vlierberghe, P. Dubruel, Protein Functionalization revised: $\mathrm{N}$-tert-butoxycarbonylation as an elegant tool to circumvent protein crosslinking, Macromol. Rapid Commun. 35 (2014) 1351-1355. doi:10.1002/marc.201400103.

[42] R. Liska, M. Schuster, R. Inführ, C. Turecek, C. Fritscher, B. Seidl, V. Schmidt, L. Kuna, a. Haase, F. Varga, H. Lichtenegger, J. Stampfl, Photopolymers for rapid prototyping, J. Coatings Technol. Res. 4 (2007) 505-510. doi:10.1007/s11998-007-9059-3.

[43] M. Schuster, C. Turecek, F. Varga, H. Lichtenegger, J. Stampfl, R. Liska, 3D-shaping of biodegradable photopolymers for hard tissue replacement, Appl. Surf. Sci. 254 (2007) 11311134. doi:10.1016/J.APSUSC.2007.07.182.

[44] Y. Cao, B.H. Lee, H.B. Peled, S.S. Venkatraman, Synthesis of stiffness-tunable and cellresponsive Gelatin-poly(ethylene glycol) hydrogel for three-dimensional cell encapsulation, J. Biomed. Mater. Res. - Part A. 104 (2016) 2401-2411. doi:10.1002/jbm.a.35779.

[45] S. Van Vlierberghe, E. Schacht, P. Dubruel, Reversible gelatin-based hydrogels: Finetuning of material properties, Eur. Polym. J. 47 (2011) 1039-1047. doi:10.1016/j.eurpolymj.2011.02.015.

[46] L. Tytgat, L. Van Damme, J. Van Hoorick, H. Declercq, H. Thienpont, H. Ottevaere, P. Blondeel, P. Dubruel, S. Van Vlierberghe, Additive manufacturing of photo-crosslinked gelatin scaffolds for adipose tissue engineering, Acta Biomater. (Accepted) (2019) 1-21.

[47] S. Duggan, O. O'Donovan, E. Owens, W. Cummins, H. Hughes, Synthesis of mucoadhesive thiolated gelatin using a two-step reaction process, Eur. J. Pharm. Biopharm. 91 (2015) 75-81. doi:10.1016/j.ejpb.2015.01.027.

[48] J. Rohrer, A. Partenhauser, O. Zupančič, G. Leonavičiūtė, S. Podričnik, A. Bernkop-Schnürch, Thiolated gelatin films: Renaissance of gelatin as sustained intraoral dosage form, Eur. Polym. J. 87 (2017) 48-59. doi:10.1016/j.eurpolymj.2016.11.028.

[49] Y. Fu, K. Xu, X. Zheng, A.J. Giacomin, A.W. Mix, W.J. Kao, 3D cell entrapment in crosslinked thiolated gelatin-poly(ethylene glycol) diacrylate hydrogels, Biomaterials. 33 (2012) 48-58. doi:10.1016/j.biomaterials.2011.09.031. 
[50] J. Rohrer, O. Zupancic, W. Suchaoin, K. Netsoomboon, F. Laffleur, S. Oh, A. Bernkop-Schnürch, Synthesis and in vitro characterisation of preactivated thiolated gelatin, Eur. Polym. J. 73 (2015) 268-277. doi:10.1016/j.eurpolymj.2015.10.023.

[51] X.Z. Shu, Y. Liu, F. Palumbo, G.D. Prestwich, Disulfide-crosslinked hyaluronan-gelatin hydrogel films: A covalent mimic of the extracellular matrix for in vitro cell growth, Biomaterials. 24 (2003) 3825-3834. doi:10.1016/S0142-9612(03)00267-9.

[52] J.M. Heffernan, D.J. Overstreet, L.D. Le, B.L. Vernon, R.W. Sirianni, Bioengineered Scaffolds for 3D Analysis of Glioblastoma Proliferation and Invasion, Ann. Biomed. Eng. 43 (2015) 19651977. doi:10.1007/s10439-014-1223-1.

[53] L. Russo, A. Sgambato, R. Visone, P. Occhetta, M. Moretti, M. Rasponi, F. Nicotra, L. Cipolla, Gelatin hydrogels via thiol-ene chemistry, Monatshefte Fur Chemie. 147 (2016) 587-592. doi:10.1007/s00706-015-1614-5.

[54] T. Mazaki, Y. Shiozaki, K. Yamane, A. Yoshida, M. Nakamura, Y. Yoshida, D. Zhou, T. Kitajima, M. Tanaka, Y. Ito, T. Ozaki, A. Matsukawa, A novel, visible light-induced, rapidly cross-linkable gelatin scaffold for osteochondral tissue engineering, Sci. Rep. 4 (2014) 1-10. doi:10.1038/srep04457.

[55] T. II Son, M. Sakuragi, S. Takahashi, S. Obuse, J. Kang, M. Fujishiro, H. Matsushita, J. Gong, S. Shimizu, Y. Tajima, Y. Yoshida, K. Suzuki, T. Yamamoto, M. Nakamura, Y. Ito, Visible lightinduced crosslinkable gelatin, Acta Biomater. 6 (2010) 4005-4010. doi:10.1016/j.actbio.2010.05.018.

[56] S. AnilKumar, S.C. Allen, N. Tasnim, T. Akter, S. Park, A. Kumar, M. Chattopadhyay, Y. Ito, L.J. Suggs, B. Joddar, The applicability of furfuryl-gelatin as a novel bioink for tissue engineering applications, J. Biomed. Mater. Res. - Part B Appl. Biomater. 107 (2019) 314-323. doi:10.1002/jbm.b.34123.

[57] S. Anil Kumar, N. Tasnim, E. Dominguez, S.C. Allen, L.J. Suggs, Y. Ito, B. Joddar, A Comparative Study of a 3D Bioprinted Gelatin-Based Lattice ans Rectangular-Sheet Structures, Gels. 4 (2018) 
1-12. doi:10.3390/gels4030073.

[58] C. García-Astrain, C. Peña-Rodriguez, A. Retegi, A. Eceiza, M.A. Corcuera, N. Gabilondo, Green chemistry for the cross-linking of photo-sensitive furan modified gelatin, Mater. Lett. 160 (2015) 142-145. doi:10.1016/j.matlet.2015.07.096.

[59] K.M. Gattás-Asfura, E. Weisman, F.M. Andreopoulos, M. Micic, B. Muller, S. Sirpal, S.M. Pham, R.M. Leblanc, Nitrocinnamate-functionalized gelatin: synthesis and "smart" hydrogel formation via photo-cross-linking., Biomacromolecules. 6 (2005) 1503-9. doi:10.1021/bm049238w.

[60] F. Yu, X. Cao, L. Zeng, Q. Zhang, X. Chen, An interpenetrating HA/G/CS biomimic hydrogel via Diels-Alder click chemistry for cartilage tissue engineering, Carbohydr. Polym. 97 (2013) 188195. doi:10.1016/j.carbpol.2013.04.046.

[61] C. García-Astrain, A. Gandini, C. Peña, I. Algar, A. Eceiza, M. Corcuera, N. Gabilondo, Diels-Alder "click" chemistry for the cross-linking of furfuryl-gelatin-polyetheramine hydrogels, RSC Adv. 4 (2014) 35578-35587. doi:10.1039/c4ra06122e.

[62] S. Bertlein, G. Brown, K.S. Lim, T. Jungst, T. Boeck, T. Blunk, J. Tessmar, G.J. Hooper, T.B.F. Woodfield, J. Groll, Thiol - Ene Clickable Gelatin: A Platform Bioink for Multiple 3D Biofabrication Technologies, Adv. Mater. 1703404 (2017) 1-6. doi:10.1002/adma.201703404.

[63] X.H. Qin, J. Torgersen, R. Saf, S. Mühleder, N. Pucher, S.C. Ligon, W. Holnthoner, H. Redl, A. Ovsianikov, J. Stampfl, R. Liska, Three-dimensional microfabrication of protein hydrogels via two-photon-excited thiol-vinyl ester photopolymerization, J. Polym. Sci. Part A Polym. Chem. 51 (2013) 4799-4810. doi:10.1002/pola.26903.

[64] J. Van Hoorick, P. Gruber, M. Markovic, M. Rollot, G. Graulus, M. Vagenende, M. Tromayer, J. Van Erps, H. Thienpont, J.C. Martins, S. Baudis, A. Ovsianikov, P. Dubruel, S. Van Vlierberghe, Highly Reactive Thiol-Norbornene Photo-Click Hydrogels : Toward Improved Processability, Macromol. Rapid Commun. 1800181 (2018) 1-7. doi:10.1002/marc.201800181.

[65] Z. Mũnoz, H. Shih, C.-C. Lin, Gelatin hydrogels formed by orthogonal thiol-norbornene photochemistry for cell encapsulation, Biomater. Sci. 2 (2014) 1063. doi:10.1039/c4bm00070f. 
[66] B. V Sridhar, E.A. Dailing, J.L. Brock, J.W. Stansbury, M.A. Randolph, K.S. Anseth, A Biosynthetic Scaffold that Facilitates Chondrocyte-Mediated Degradation and Promotes Articular Cartilage Extracellular Matrix Deposition, Regen. Eng. Transl. Med. 1 (2015) 11-21. doi:10.1007/s40883015-0002-3.

[67] O. Bas, E.M. De-Juan-Pardo, M.P. Chhaya, F.M. Wunner, J.E. Jeon, T.J. Klein, D.W. Hutmacher, Enhancing structural integrity of hydrogels by using highly organised melt electrospun fibre constructs, Eur. Polym. J. 72 (2015) 451-463. doi:10.1016/j.eurpolymj.2015.07.034.

[68] J. Visser, F.P.W. Melchels, J.E. Jeon, E.M. van Bussel, L.S. Kimpton, H.M. Byrne, W.J. a Dhert, P.D. Dalton, D.W. Hutmacher, J. Malda, Reinforcement of hydrogels using three-dimensionally printed microfibres., Nat. Commun. 6 (2015) 6933. doi:10.1038/ncomms7933.

[69] C. Claaßen, M.H. Claaßen, V. Tru, L. Sewald, G.E.M. Tovar, K. Borchers, A. Southan, Quantification of Substitution of Gelatin Methacryloyl: Best Practice and Current Pitfalls, Biomacromolecules. 19 (2018) 42-52. doi:10.1021/acs.biomac.7b01221.

[70] A. Houben, N. Pien, X. Lu, F. Bisi, J. Van Hoorick, M.N. Boone, P. Roose, H. Van den Bergen, D. Bontinck, T. Bowden, P. Dubruel, S. Van Vlierberghe, Indirect Solid Freeform Fabrication of an Initiator-Free Photocrosslinkable Hydrogel Precursor for the Creation of Porous Scaffolds, Macromol. Biosci. (2016) 1-12. doi:10.1002/mabi.201600289.

[71] B. Sara Žigon-Branc, M. Markovic, J. Van Hoorick, S. Van Vlierberghe, P. Dubruel, E. Zerobin, S. Baudis, A. Ovsianikov, S. Žigon-Branc, Impact of hydrogel stiffness on differentiation of human adipose-derived stem cell microspheroids, Tissue Eng. Part A. 43 (2018) 1-40. doi:10.1089/ten.TEA.2018.0237.

[72] Y.-C. Chen, R.-Z. Lin, H. Qi, Y. Yang, H. Bae, J.M. Melero-Martin, A. Khademhosseini, Functional Human Vascular Network Generated in Photocrosslinkable Gelatin Methacrylate Hydrogels, Adv. Funct. Mater. 22 (2012) 2027-2039. doi:10.1002/adfm.201101662.

[73] X. Zhao, Q. Lang, L. Yildirimer, Z.Y. Lin, W. Cui, N. Annabi, K.W. Ng, M.R. Dokmeci, A.M. Ghaemmaghami, A. Khademhosseini, Photocrosslinkable Gelatin Hydrogel for Epidermal 
Tissue Engineering, Adv. Healthc. Mater. 5 (2016) 108-118. doi:10.1002/adhm.201500005.

[74] J.W. Nichol, S.T. Koshy, H. Bae, C.M. Hwang, S. Yamanlar, A. Khademhosseini, Cell-laden microengineered gelatin methacrylate hydrogels., Biomaterials. 31 (2010) 5536-44. doi:10.1016/j.biomaterials.2010.03.064.

[75] A. Mignon, D. Pezzoli, E. Prouvé, L. Lévesque, A. Arslan, N. Pien, D. Schaubroeck, D. Mantovani, S. Van Vlierberghe, P. Dubruel, Combined effect of Laponite and polymer molecular weight on the cell-interactive properties of synthetic PEO-based hydrogels. submitted., React. Funct. Polym. 136 (2019) 95-106. doi:10.1016/j.reactfunctpolym.2018.12.017.

[76] D. Mandt, P. Gruber, M. Markovic, M. Tromayer, M. Rothbauer, S. Rudi, A. Kratz, S.F. Ali, J. Van Hoorick, W. Holnthoner, S. Mühleder, P. Dubruel, S. Van Vlierberghe, P. Ertl, R. Liska, A. Ovsianikov, Fabrication of biomimetic placental barrier structures within a microfluidic device utilizing two-photon polymerization, Int. J. Bioprining. 4 (2018) 1-12. doi:10.18063/ijb.v4i2.144.

[77] M.-H. Chang, Y.-P. Hsiao, C.-Y. Hsu, P.-S. Lai, Photo-Crosslinked Polymeric Matrix with Antimicrobial Functions for Excisional Wound Healing in Mice, Nanomaterials. 8 (2018) 791. doi:10.3390/nano8100791.

[78] R. Stevens, L. Stevens, N.C. Price, The Stabilities of Various Thiol Compounds used in Protein Purifications, in: Biochem. Educ. 11, 1983: p. 70.

[79] A.L. Freeman, G.R. Buttermann, B.P. Beaubien, W.E. Rochefort, Compressive properties of fibrous repair tissue compared to nucleus and annulus., J. Biomech. 46 (2013) 1714-21. doi:10.1016/j.jbiomech.2013.03.034.

[80] R.F. Pereira, P.J. Bartolo, 3D Bioprinting of photocrosslinkable hydrogel constructs, J. Appl. Polym. Sci. 1 (2015). doi:10.1002/app.42889.

[81] C.E. Hoyle, C.N. Bowman, Thiol - Ene Click Chemistry, Angew. Chemie. 49 (2010) 1540-1573. doi:10.1002/anie.200903924.

[82] K.S. Lim, B.S. Schon, N. V. Mekhileri, G.C.J. Brown, C.M. Chia, S. Prabakar, G.J. Hooper, T.B.F. 
Woodfield, New Visible-Light Photoinitiating System for Improved Print Fidelity in GelatinBased Bioinks, ACS Biomater. Sci. Eng. 2 (2016) 1752-1762. doi:10.1021/acsbiomaterials.6b00149.

[83] H.C. Kolb, M.G. Finn, K.B. Sharpless, Click Chemistry: Diverse Chemical Function from a Few Good Reactions, Angew. Chemie - Int. Ed. 40 (2001) 2004-2021. doi:10.1002/15213773(20010601)40:11<2004::AID-ANIE2004>3.3.CO;2-X.

[84] T.O. Machado, C. Sayer, P.H.H. Araujo, Thiol-ene polymerisation: A promising technique to obtain novel biomaterials, Eur. Polym. J. 86 (2017) 200-215. doi:10.1016/j.eurpolymj.2016.02.025.

[85] C.E. Hoyle, T.A.I.Y. Lee, T. Roper, Thiol - Enes: Chemistry of the Past with Promise for the Future, J. Polym. Sci. Part A Polym. Chem. 42 (2004) 5301-5338. doi:10.1002/pola.20366.

[86] L. Li, C. Lu, L. Wang, M. Chen, J. White, X. Hao, K.M. McLean, H. Chen, T.C. Hughes, GelatinBased Photocurable Hydrogels for Corneal Wound Repair, ACS Appl. Mater. Interfaces. 10 (2018) 13283-13292. doi:10.1021/acsami.7b17054.

[87] M.M. Perera, N. Ayres, Gelatin based dynamic hydrogels via thiol-norbornene reactions, Polym. Chem. 8 (2017) 6741-6749. doi:10.1039/c7py01630a.

[88] K. Xu, Y. Fu, W. Chung, X. Zheng, Y. Cui, I.C. Hsu, W.J. Kao, Thiol-ene-based biological/synthetic hybrid biomatrix for 3-D living cell culture, Acta Biomater. 8 (2012) 2504-2516. doi:10.1016/j.actbio.2012.03.049.Thiol.

[89] Y. Dong, A. Sigen, M. Rodrigues, X. Li, S.H. Kwon, N. Kosaric, S. Khong, Y. Gao, W. Wang, G.C. Gurtner, Injectable and Tunable Gelatin Hydrogels Enhance Stem Cell Retention and Improve Cutaneous Wound Healing, Adv. Funct. Mater. 27 (2017) 1-12. doi:10.1002/adfm.201606619.

[90] C.-C. Lin, C.S. Ki, H. Shih, Thiol-norbornene photo-click hydrogels for tissue engineering applications., J. Appl. Polym. Sci. 132 (2015) 1-11. doi:10.1002/app.41563.

[91] C. Resetco, B. Hendriks, N. Badi, F. Du Prez, Thiol-ene chemistry for polymer coatings and surface modification-building in sustainability and performance, Mater. Horizons. 4 (2017) 
1041-1053. doi:10.1039/c7mh00488e.

[92] J.D. Mccall, K.S. Anseth, Thiol - Ene Photopolymerizations Provide a Facile Method To Encapsulate Proteins and Maintain Their Bioactivity, (2012).

[93] A. Dobos, W. Steiger, D. Theiner, P. Gruber, M. Lunzer, J. Van Hoorick, S. Van Vlierberghe, A. Ovsianikov, Screening of two-photon activated photodynamic therapy sensitizers using a 3D osteosarcoma model, Analyst. (2019). doi:10.1039/C9AN00068B.

[94] D.L. Alge, M.A. Azagarsamy, D.F. Donohue, K.S. Anseth, Synthetically tractable click hydrogels for three-dimensional cell culture formed using tetrazine-norbornene chemistry, Biomacromolecules. 14 (2013) 949-953. doi:10.1021/bm4000508.

[95] W.M. Gramlich, I.L. Kim, J.A. Burdick, Synthesis and orthogonal photopatterning of hyaluronic acid hydrogels with thiol-norbornene chemistry, Biomaterials. 34 (2013) 9803-9811. doi:10.1016/j.biomaterials.2013.08.089.

[96] R.F. Pereira, P.J. Bártolo, 3D Photo-Fabrication for Tissue Engineering and Drug Delivery, Engineering. 1 (2015) 090-112. doi:10.15302/J-ENG-2015015.

[97] C.F. Hansell, P. Espeel, M.M. Stamenovi??, I.A. Barker, A.P. Dove, F.E. Du Prez, R.K. Oreilly, Additive-free clicking for polymer functionalization and coupling by tetrazine-norbornene chemistry, J. Am. Chem. Soc. 133 (2011) 13828-13831. doi:10.1021/ja203957h.

[98] F. Goethals, D. Frank, F. Du Prez, Protected thiol strategies in macromolecular design, Prog. Polym. Sci. 64 (2017) 76-113. doi:10.1016/j.progpolymsci.2016.09.003.

[99] M. Gajendiran, J.-S. Rhee, K. Kim, Recent Developments in Thiolated Polymeric Hydrogels for Tissue Engineering Applications, Tissue Eng. Part B Rev. 24 (2017) ten.TEB.2016.0442. doi:10.1089/ten.TEB.2016.0442.

[100] S.T. Koshy, R.M. Desai, P. Joly, J. Li, R.K. Bagrodia, S.A. Lewin, N.S. Joshi, D.J. Mooney, ClickCrosslinked Injectable Gelatin Hydrogels, Adv. Healthc. Mater. 5 (2016) 541-547. doi:10.1002/adhm.201500757.

[101] E. Hoch, C. Schuh, T. Hirth, G.E.M. Tovar, K. Borchers, Stiff gelatin hydrogels can be photo- 
chemically synthesized from low viscous gelatin solutions using molecularly functionalized gelatin with a high degree of methacrylation., J. Mater. Sci. Mater. Med. 23 (2012) 2607-17. doi:10.1007/s10856-012-4731-2.

[102] D.P. Nair, M. Podgórski, S. Chatani, T. Gong, W. Xi, C.R. Fenoli, C.N. Bowman, The Thiol-Michael addition click reaction: A powerful and widely used tool in materials chemistry, Chem. Mater. 26 (2013) 724-744. doi:10.1021/cm402180t.

[103] Y. Jiang, J. Chen, C. Deng, E.J. Suuronen, Z. Zhong, Click hydrogels, microgels and nanogels: Emerging platforms for drug delivery and tissue engineering, Biomaterials. 35 (2014) 49694985. doi:10.1016/j.biomaterials.2014.03.001.

[104] C. García-Astrain, O. Guaresti, K. González, A. Eceiza, M.A. Corcuera, N. Gabilondo, Click gelatin hydrogels : Characterization and drug release behaviour, Mater. Lett. 182 (2016) 134-137. doi:10.1016/j.matlet.2016.06.115.

[105] A.N. Zelikin, J.F. Quinn, F. Caruso, Disulfide cross-linked polymer capsules: En route to biodeconstructible systems, Biomacromolecules. 7 (2006) 27-30. doi:10.1021/bm050832v.

[106] B. Gyarmati, Á. Némethy, A. Szilágyi, Reversible disulphide formation in polymer networks: A versatile functional group from synthesis to applications, Eur. Polym. J. 49 (2013) 1268-1286. doi:10.1016/j.eurpolymj.2013.03.001.

[107] S. Kommareddy, M. Amiji, Preparation and evaluation of thiol-modified gelatin nanoparticles for intracellular DNA delivery in response to glutathione, Bioconjug. Chem. 16 (2005) 14231432. doi:10.1021/bc050146t.

[108] A. Bernkop-Schnürch, Thiomers: A new generation of mucoadhesive polymers, Adv. Drug Deliv. Rev. 57 (2005) 1569-1582. doi:10.1016/j.addr.2005.07.002.

[109] T. Seki, H. Kanbayashi, T. Nagao, S. Chono, M. Tomita, M. Hayashi, Y. Tabata, K. Morimoto, Effect of aminated gelatin on the nasal absorption of insulin in rats., Biol. Pharm. Bull. 28 (2005) 510-4. doi:10.1248/bpb.28.510.

[110] A.J. Kowaltowski, A.E. Vercesi, Mitochondrial Damage Induced By Conditions of Oxidative 
Stress, Free Radic. Biol. Med. 26 (1998) 463-471. papers2://publication/uuid/A2D9860B-98814635-BE65-833E1FB6FFA0.

[111] B. Sarker, D.G. Papageorgiou, R. Silva, T. Zehnder, F. Gul-E-Noor, M. Bertmer, J. Kaschta, K. Chrissafis, R. Detsch, A.R. Boccaccini, Fabrication of alginate-gelatin crosslinked hydrogel microcapsules and evaluation of the microstructure and physico-chemical properties, J. Mater. Chem. B. 2 (2014) 1470-1482. doi:10.1039/c3tb21509a.

[112] Y. Liu, M.B. Chan-Park, Hydrogel based on interpenetrating polymer networks of dextran and gelatin for vascular tissue engineering, Biomaterials. $30 \quad$ (2009) 196-207. doi:10.1016/j.biomaterials.2008.09.041.

[113] Y. Liu, M.B. Chan-Park, Hydrogel based on interpenetrating polymer networks of dextran and gelatin for vascular tissue engineering, Biomaterials. $30 \quad$ (2009) $\quad 196-207$. doi:10.1016/j.biomaterials.2008.09.041.

[114] S.H. Medina, J.P. Schneider, Chemical Ligations in the Design of Hydrogel Materials, in: W.R. Algar, P.E. Dawson, I.L. Medintz (Eds.), Chemoselective Bioorthogonal Ligation React., 2017: pp. 497-542. doi:10.1002/9783527683451.ch16.

[115] L.A. Wells, M.A. Brook, H. Sheardown, Generic, anthracene-based hydrogel crosslinkers for photo-controllable drug delivery, Macromol. Biosci. $11 \quad$ (2011) 988-998. doi:10.1002/mabi.201100001.

[116] S. Sirpal, K.M. Gattás-Asfura, R.M. Leblanc, A photodimerization approach to crosslink and functionalize microgels, Colloids Surfaces B Biointerfaces. 58 (2007) 116-120. doi:10.1016/j.colsurfb.2007.02.016.

[117] K.M.C. Tsang, N. Annabi, F. Ercole, K. Zhou, D.J. Karst, F. Li, J.M. Haynes, R.A. Evans, H. Thissen, A. Khademhosseini, J.S. Forsythe, Facile one-step micropatterning using photodegradable gelatin hydrogels for improved cardiomyocyte organization and alignment, Adv. Funct. Mater. 25 (2015) 977-986. doi:10.1002/adfm.201403124.

[118] M. Lunzer, L. Shi, O.G. Andriotis, P. Gruber, M. Markovic, P.J. Thurner, D. Ossipov, R. Liska, A. 
Ovsianikov, A Modular Approach to Sensitized Two-Photon Patterning of Photodegradable Hydrogels Angewandte, (2018) 15122-15127. doi:10.1002/anie.201808908.

[119] N.N. Ferreira, L.M.B. Ferreira, V.M.O. Cardoso, F.I. Boni, A.L.R. Souza, M.P.D. Gremião, Recent advances in smart hydrogels for biomedical applications: From self-assembly to functional approaches, Eur. Polym. J. 99 (2018) 117-133. doi:10.1016/j.eurpolymj.2017.12.004.

[120] A.M. Braun, H. Dahn, E. Gassmannt, L. Jakobsl, J. Katevall, C.G. Martinez, E. Oliverosl, (2+4)Cycloaddition with singlet oxygen. 170-investigation of the reactivity of Furfuryl alcohol endoperoxide., Photochem. Photobiol. 70 (1999) 868-874.

[121] Y. Tajima, H. Arai, Y. Tezuka, T. Ishii, K. Takeuchi, Photochemical reaction of furans in the presence of [60] fullerene, Fuller. Sci. Technol. 5 (1997) 1531-1544. doi:10.1080/15363839708013336.

[122] M. Yao, J. Zhang, F. Gao, Y. Chen, S. Ma, K. Zhang, H. Liu, F. Guan, New BMSC-Laden Gelatin Hydrogel Formed in Situ by Dual- Enzymatic Cross-Linking Accelerates Dermal Wound Healing, ACS Omega. 4 (2019) 8334-8340. doi:10.1021/acsomega.9b00878.

[123] H. Degn, Bistability caused by Substrate Inhibition of Peroxidase in an Open Reaction System, Nature. 217 (1968).

[124] S. Sakai, H. Ohi, T. Hotta, H. Kamei, M. Taya, Differentiation potential of human adipose stem cells bioprinted with hyaluronic acid/gelatin-based bioink through microextrusion and visible light-initiated crosslinking, Biopolymers. 109 (2018) 1-8. doi:10.1002/bip.23080.

[125] R.G.W. Norrish, C.H. Bamfore, Photo-decomposition of Aldehydes and Ketones, Nature. 140 (1937) 195-196.

[126] K.S. Lim, B.J. Klotz, G.C.J. Lindberg, F.P.W. Melchels, G.J. Hooper, J. Malda, D. Gawlitta, T.B.F. Woodfield, Visible Light Cross-Linking of Gelatin Hydrogels Offers an Enhanced Cell Microenvironment with Improved Light Penetration Depth, Macromol. Biosci. (2019) 1-14. doi:10.1002/mabi.201900098.

[127] M. Tromayer, A. Dobos, P. Gruber, A. Ajami, R. Dedic, A. Ovsianikov, R. Liska, A biocompatible 
diazosulfonate initiator for direct encapsulation of human stem cells via two-photon polymerization, Polym. Chem. 9 (2018) 3108-3117. doi:10.1039/C8PY00278A.

[128] D.A. Fancy, C. Denison, K. Kom, Y. Xie, T. Holdeman, F. Amini, T. Kodadek, Scope, limitations and mechanistic aspects of the photo-induced cross-linking of proteins by water-soluble metal complexes, Chem. Biol. 7 (2000) 697-708.

[129] Z. Li, J. Torgersen, A. Ajami, S. Mühleder, X. Qin, W. Husinsky, W. Holnthoner, A. Ovsianikov, J. Stampfl, R. Liska, Initiation efficiency and cytotoxicity of novel water-soluble two-photon photoinitiators for direct 3D microfabrication of hydrogels, RSC Adv. 3 (2013) 15939. doi:10.1039/c3ra42918k.

[130] L. Brigo, A. Urciuolo, S. Giulitti, G. Della Giustina, M. Tromayer, R. Liska, N. Elvassore, G. Brusatin, 3D high-resolution two-photon crosslinked hydrogel structures for biological studies, Acta Biomater. 55 (2017) 373-384. doi:10.1016/j.actbio.2017.03.036.

[131] X. Wang, X.H. Qin, C. Hu, A. Terzopoulou, X.Z. Chen, T.Y. Huang, K. Maniura-Weber, S. Pané, B.J. Nelson, 3D Printed Enzymatically Biodegradable Soft Helical Microswimmers, Adv. Funct. Mater. 28 (2018) 1-8. doi:10.1002/adfm.201804107.

[132] M. Tromayer, P. Gruber, M. Markovic, A. Rosspeintner, E. Vauthey, H. Redl, A. Ovsianikov, R. Liska, A biocompatible macromolecular two-photon initiator based on hyaluronan, Polym. Chem. 8 (2017) 451-460. doi:10.1039/C6PY01787H.

[133] J.E. Mark, Polymer Data Handbook, 1999. doi:10.1021/ja907879q.

[134] M. Schuster, C. Turecek, G. Weigel, R. Saf, J. Stampfl, F. Varga, R. Liska, Gelatin-Based Photopolymers for Bone Replacement Materials, J. Polym. Sci. Part A Polym. Chem. 47 (2009) 7078-7089. doi:10.1002/pola.

[135] R. Zamani Alavijeh, P. Shokrollahi, J. Barzin, A thermally and water activated shape memory gelatin physical hydrogel, with a gel point above the physiological temperature, for biomedical applications, J. Mater. Chem. B. 5 (2017) 2302-2314. doi:10.1039/c7tb00014f.

[136] J.R. Prado, S. Vyazovkin, Melting of Gelatin Gels Containing Laponite, Montmorillonite, and 
Chhitosan Particles, Macromol. Chem. Phys. $215 \quad$ (2014) 867-872. doi:10.1002/macp.201400029.

[137] P. De Meuter, H. Rahier, B. Van Mele, The use of modulated temperature differential scanning calorimetry for the characterisation of food systems, Int. J. Pharm. 192 (1999) 77-84. doi:10.1016/S0378-5173(99)00274-4.

[138] E. Hoch, T. Hirth, G.E.M. Tovar, K. Borchers, Chemical tailoring of gelatin to adjust its chemical and physical properties for functional bioprinting, J. Mater. Chem. B. 1 (2013) 5675-5685. doi:10.1039/c3tb20745e.

[139] L. Rebers, T. Granse, G. Tovar, A. Southan, K. Borchers, Physical Interactions Strengthen Chemical Gelatin Methacryloyl Gels, Gels. 5 (2019) 4. doi:10.3390/gels5010004.

[140] L. Rebers, T. Granse, G. Tovar, A. Southan, K. Borchers, Physical Interactions Strengthen Chemical Gelatin Methacryloyl Gels, Gels. 5 (2019) 4. doi:10.3390/gels5010004.

[141] B. Sen Chiou, R.J. Avena-Bustillos, P.J. Bechtel, H. Jafri, R. Narayan, S.H. Imam, G.M. Glenn, W.J. Orts, Cold water fish gelatin films: Effects of cross-linking on thermal, mechanical, barrier, and biodegradation properties, Eur. Polym. J. 44 (2008) 3748-3753. doi:10.1016/j.eurpolymj.2008.08.011.

[142] A.A. Karim, R. Bhat, Gelatin alternatives for the food industry: recent developments, challenges and prospects, Trends Food Sci. Technol. 19 (2008) 644-656. doi:10.1016/j.tifs.2008.08.001.

[143] E. Hoch, K. Borchers, G.E.M. Tovar, Modifizierte Gekatine, Vefahren zu ihrer Herstellung unf Verwending, DE 102012219691 A1 2014.04.30, 2012.

[144] S. Ma, M. Natoli, X. Liu, M.P. Neubauer, F.M. Watt, A. Fery, W.T.S. Huck, Monodisperse collagen-gelatin beads as potential platforms for 3D cell culturing, J. Mater. Chem. B. 1 (2013) 5128. doi:10.1039/c3tb20851f.

[145] C.D. O'Connell, B. Zhang, C. Onofrillo, S. Duchi, R. Blanchard, A. Quigley, J. Bourke, S. Gambhir, R. Kapsa, C. Di Bella, P. Choong, G.G. Wallace, Tailoring the mechanical properties of gelatin methacryloyl hydrogels through manipulation of the photocrosslinking conditions, Soft Matter. 
14 (2018) 2142-2151. doi:10.1039/C7SM02187A.

[146] W. Schuurman, P.A. Levett, M.W. Pot, P.R. van Weeren, W.J.A. Dhert, D.W. Hutmacher, F.P.W. Melchels, T.J. Klein, J. Malda, Gelatin-methacrylamide hydrogels as potential biomaterials for fabrication of tissue-engineered cartilage constructs, Macromol. Biosci. 13 (2013) 551-561. doi:10.1002/mabi.201200471.

[147] J. Malda, J. Visser, F.P. Melchels, T. Jüngst, W.E. Hennink, W.J.A. Dhert, J. Groll, D.W. Hutmacher, 25th anniversary article: Engineering hydrogels for biofabrication, Adv. Mater. 25 (2013) 5011-5028. doi:10.1002/adma.201302042.

[148] L. Tytgat, M. Vagenende, H. Declercq, J.C. Martins, H. Thienpont, H. Ottevaere, Synergistic effect of k-carrageenan and gelatin blends towards adipose tissue engineering, Carbohydr. Polym. 189 (2018) 1-9. doi:10.1016/j.carbpol.2018.02.002.

[149] M.F. Butler, M. Heppenstall-Butler, "Delayed" Phase Separation in a Gelatin/Dextran Mixture Studied by Small-Angle Light Scattering, Turbidity, Confocal Laser Scanning Microscopy, and Polarimetry, Biomacromolecules. 4 (2003) 928-936. doi:10.1021/BM0340319.

[150] W. Zhu, H. Cui, B. Boualam, F. Masood, E. Flynn, R.D. Rao, Z.Y. Zhang, L.G. Zhang, 3D bioprinting mesenchymal stem cell-laden construct with core-shell nanospheres for cartilage tissue engineering, Nanotechnology. 29 (2018). doi:10.1088/1361-6528/aaafa1.

[151] W. Jia, P.S. Gungor-Ozkerim, Y.S. Zhang, K. Yue, K. Zhu, W. Liu, Q. Pi, B. Byambaa, M.R. Dokmeci, S.R. Shin, A. Khademhosseini, Direct 3D bioprinting of perfusable vascular constructs using a blend bioink, Biomaterials. 106 (2016) 58-68. doi:10.1016/j.biomaterials.2016.07.038.

[152] O. Bas, E.M. De-Juan-Pardo, M.P. Chhaya, F.M. Wunner, J.E. Jeon, T.J. Klein, D.W. Hutmacher, Enhancing structural integrity of hydrogels by using highly organised melt electrospun fibre constructs, Eur. Polym. J. 72 (2015) 451-463. doi:10.1016/j.eurpolymj.2015.07.034.

[153] X. Hu, L. Ma, C. Wang, C. Gao, Gelatin hydrogel prepared by photo-initiated polymerization and loaded with TGF-beta1 for cartilage tissue engineering., Macromol. Biosci. 9 (2009) 1194-201. doi:10.1002/mabi.200900275. 
[154] S. Van Vlierberghe, S.K. Samal, P. Dubruel, Development of Mechanically Tailored GelatinChondroitin Sulphate Hydrogel Films, Macromol. Symp. 309-310 (2011) 173-181. doi:10.1002/masy.201100030.

[155] C.S. Nickerson, J. Park, J.A. Kornfield, H. Karageozian, Rheological properties of the vitreous and the role of hyaluronic acid, J. Biomech. 41 (2008) 1840-1846. doi:10.1016/j.jbiomech.2008.04.015.

[156] K. Comley, N.A. Fleck, The toughness of adipose tissue : measurements and physical basis, J. Biomech. 43 (2010) 1823-1826. doi:10.1016/j.jbiomech.2010.02.029.

[157] K. Comley, N.A. Fleck, International Journal of Solids and Structures A micromechanical model for the Young ' s modulus of adipose tissue, Int. J. Solids Struct. 47 (2010) 2982-2990. doi:10.1016/j.ijsolstr.2010.07.001.

[158] K.-H. Chang, H.-T. Liao, J.-P. Chen, Preparation and characterization of gelatin/hyaluronic acid cryogels for adipose tissue engineering: in vitro and in vivo studies., Acta Biomater. 9 (2013) 9012-26. doi:10.1016/j.actbio.2013.06.046.

[159] R.J. Dewall, T. Varghese, M.A. Kliewer, J.M. Harter, M. Hartenbach, Compression-Dependent Viscoelastic behavior of human cervix tissue, Ultrason Imaging. 32 (2011) 214-228.

[160] S. Cheng, E.C. Clarke, L.E. Bilston, Rheological properties of the tissues of the central nervous system: A review, Med. Eng. Phys. 30 (2008) 1318-1337. doi:10.1016/j.medengphy.2008.06.003.

[161] M. Zhang, P. Nigwekar, B. Castaneda, K. Hoyt, J. V. Joseph, A. Di Sant' Agnese, E.M. Messing, J.G. Strang, D.J. Rubens, K.J. Parker, Quantitative Characterization of viscoelastic properties of human prostate correlated with histology, Ultrasound Med. Biol. 34 (2008) 1033-1042. doi:10.1016/j.ultrasmedbio.2007.11.024.

[162] H. Frank, A.J. Grodzinsky, MODEL OF CARTILAGE ELECTROKINETICS AND, 20 (1987) 629-639.

[163] H. Wang, P.L. Prendiville, P.J. McDonnell, W. V. Chang, An ultrasonic technique for the measurement of the elastic moduli of human cornea, J. Biomech. 29 (1996) 1633-1636. 
[164] T.K.L. Meyvis, B.G. Stubbe, M.J. Van Steenbergen, W.E. Hennink, S.C. De Smedt, J. Demeester, A comparison between the use of dynamic mechanical analysis and oscillatory shear rheometry for the characterisation of hydrogels, Int. J. Pharm. 244 (2002) 163-168.

[165] A. Houben, J. Van Hoorick, J. Van Erps, H. Thienpont, S. Van Vlierberghe, P. Dubruel, Indirect Rapid Prototyping: Opening Up Unprecedented Opportunities in Scaffold Design and Applications, Ann. Biomed. Eng. 45 (2016) 1-26. doi:10.1007/s10439-016-1610-x.

[166] E. De Jaeghere, E. De Vlieghere, J. Van Hoorick, S. Van Vlierberghe, G. Wagemans, L. Pieters, E. Melsens, M. Praet, J. Van Dorpe, M.N. Boone, R. Ghobeira, N. De Geyter, M. Bracke, C. Vanhove, S. Neyt, G. Berx, B.G. De Geest, P. Dubruel, H. Declercq, W. Ceelen, O. De Wever, Heterocellular 3D scaffolds as biomimetic to recapitulate the tumor microenvironment of peritoneal metastases in vitro and in vivo, Biomaterials. 158 (2018) 95-105. doi:10.1016/j.biomaterials.2017.12.017.

[167] E. Van De Walle, I. Van Nieuwenhove, E. Vanderleyden, H. Declercq, K. Gellynck, D. Schaubroeck, H. Ottevaere, H. Thienpont, W.H. De Vos, M. Cornelissen, S. Van Vlierberghe, P. Dubruel, Polydopamine-gelatin as universal cell-interactive coating for methacrylate-based medical device packaging materials: When surface chemistry overrules substrate bulk properties, Biomacromolecules. 17 (2016) 56-68. doi:10.1021/acs.biomac.5b01094.

[168] T. Xu, C.A. Gregory, P. Molnar, X. Cui, S. Jalota, S.B. Bhaduri, T. Boland, Viability and electrophysiology of neural cell structures generated by the inkjet printing method, 27 (2006) 3580-3588. doi:10.1016/j.biomaterials.2006.01.048.

[169] M. Nakamura, A. Kobayashi, F. Takagi, A. Watanabe, Y. Hiruma, K. Ohuchi, Y. Iwasaki, M. Horie, I. Morita, S. Takatani, Biocompatible Inkjet Printing Technique for Designed Seeding of Individual Living Cells, 11 (2005) 1658-1666.

[170] J. Visser, B. Peters, T.J. Burger, J. Boomstra, W.J.A. Dhert, F.P.W. Melchels, J. Malda, Biofabrication of multi-material anatomically shaped tissue constructs, Biofabrication. 5 (2013). doi:10.1088/1758-5082/5/3/035007. 
[171] T. Billiet, M. Vandenhaute, J. Schelfhout, S. Van Vlierberghe, P. Dubruel, A review of trends and limitations in hydrogel-rapid prototyping for tissue engineering., Biomaterials. 33 (2012) 60206041. doi:10.1016/j.biomaterials.2012.04.050.

[172] J. Groll, J.A. Burdick, D. Cho, B. Derby, M. Gelinsky, S.C. Heilshorn, T. Jüngst, J. Malda, A definition of bioinks and their distinction from biomaterial inks, Biofabrication. 11 (2018). doi:10.1088/1758-5090/aaec52.

[173] W. Liu, M.A. Heinrich, Y. Zhou, A. Akpek, N. Hu, X. Liu, X. Guan, Z. Zhong, X. Jin, A. Khademhosseini, Y.S. Zhang, Extrusion Bioprinting of Shear-Thinning Gelatin Methacryloyl Bioinks, Adv. Healthc. Mater. 6 (2017) 1-11. doi:10.1002/adhm.201601451.

[174] Y. Gu, L. Zhang, X. Du, Z. Fan, L. Wang, W. Sun, Y. Cheng, Y. Zhu, C. Chen, Reversible physical crosslinking strategy with optimal temperature for 3D bioprinting of human chondrocyte-laden gelatin methacryloyl bioink, J. Biomater. Appl. 33 (2018) 609-618. doi:10.1177/0885328218805864.

[175] J. Yin, M. Yan, Y. Wang, J. Fu, H. Suo, 3D Bioprinting of Low-Concentration Cell-Laden Gelatin Methacrylate (GeIMA) Bioinks with a Two-Step Cross-linking Strategy, ACS Appl. Mater. Interfaces. 10 (2018) 6849-6857. doi:10.1021/acsami.7b16059.

[176] F.P.W. Melchels, W.J.A. Dhert, D.W. Hutmacher, J. Malda, Development and characterisation of a new bioink for additive tissue manufacturing, J. Mater. Chem. B. 2 (2014) 2282-2289. doi:10.1039/c3tb21280g.

[177] R. Levato, W.R. Webb, I.A. Otto, A. Mensinga, Y. Zhang, M. van Rijen, R. van Weeren, I.M. Khan, J. Malda, The bio in the ink: cartilage regeneration with bioprintable hydrogels and articular cartilage-derived progenitor cells, Acta Biomater. 61 (2017) 41-53. doi:10.1016/j.actbio.2017.08.005.

[178] L. Ouyang, C.B. Highley, W. Sun, J.A. Burdick, COMMUNICATION A Generalizable Strategy for the 3D Bioprinting of Hydrogels from Nonviscous Photo-crosslinkable Inks, (2017). doi:10.1002/adma.201604983. 
[179] L.E. Bertassoni, J.C. Cardoso, V. Manoharan, A.L. Cristino, N.S. Bhise, W.A. Araujo, P. Zorlutuna, N.E. Vrana, A.M. Ghaemmaghami, M.R. Dokmeci, A. Khademhosseini, Direct-write bioprinting of cell-laden methacrylated gelatin hydrogels, Biofabrication. 6 (2014). doi:10.1088/1758$5082 / 6 / 2 / 024105$.

[180] F. Ruther, T. Distler, A.R. Boccaccini, R. Detsch, Biofabrication of vessel-like structures with alginate di-aldehyde-gelatin (ADA-GEL) bioink, J. Mater. Sci. Mater. Med. 30 (2019) 8. doi:10.1007/s10856-018-6205-7.

[181] D. Qi, S. Wu, M.A. Kuss, W. Shi, S. Chung, P.T. Deegan, A. Kamenskiy, Y. He, B. Duan, Mechanically robust cryogels with injectability and bioprinting supportability for adipose tissue engineering, Acta Biomater. 74 (2018) 131-142. doi:10.1016/j.actbio.2018.05.044.

[182] C. Colosi, S.R. Shin, V. Manoharan, S. Massa, M. Costantini, A. Barbetta, M.R. Dokmeci, M. Dentini, A. Khademhosseini, Microfluidic Bioprinting of Heterogeneous 3D Tissue Constructs Using Low-Viscosity Bioink, Adv. Mater. 28 (2016) 677-684a. doi:10.1002/adma.201503310.

[183] A. Skardal, J. Zhang, L. McCoard, X. Xu, S. Oottamasathien, G.D. Prestwich, Photocrosslinkable Hyaluronan-Gelatin Hydrogels for Two-Step Bioprinting, Tissue Eng. Part A. 16 (2010) 26752685. doi:10.1089/ten.tea.2009.0798.

[184] M. Yan, P.L. Lewis, R.N. Shah, Tailoring nanostructure and bioactivity of 3D-printable hydrogels with self-assemble peptides amphiphile (PA) for promoting bile duct formation, Biofabrication. 10 (2018). doi:10.1088/1758-5090/aac902.

[185] X. Zhou, N.J. Castro, W. Zhu, H. Cui, M. Aliabouzar, K. Sarkar, L.G. Zhang, Improved Human Bone Marrow Mesenchymal Stem Cell Osteogenesis in 3D Bioprinted Tissue Scaffolds with Low Intensity Pulsed Ultrasound Stimulation, Sci. Rep. 6 (2016) 1-12. doi:10.1038/srep32876.

[186] R. Gauvin, Y.C. Chen, J.W. Lee, P. Soman, P. Zorlutuna, J.W. Nichol, H. Bae, S. Chen, A. Khademhosseini, Microfabrication of complex porous tissue engineering scaffolds using 3D projection stereolithography, Biomaterials. $33 \quad$ (2012) 3824-3834. doi:10.1016/j.biomaterials.2012.01.048. 
[187] S.P. Grogan, P.H. Chung, P. Soman, P. Chen, M.K. Lotz, S. Chen, D.D. D'Lima, Digital micromirror device projection printing system for meniscus tissue engineering, Acta Biomater. 9 (2013) 7218-7226. doi:10.1016/j.actbio.2013.03.020.

[188] Z. Wang, R. Abdulla, B. Parker, R. Samanipour, S. Ghosh, K. Kim, A simple and high-resolution stereolithography-based 3D bioprinting system using visible light crosslinkable bioinks, Biofabrication. 7 (2015). doi:10.1088/1758-5090/7/4/045009.

[189] L.J. Hornbeck, Digital Light Processing for high-brightness high-resolution applications, 3013 (1997) 27-40. doi:10.1117/12.273880.

[190] P. Soman, P.H. Chung, A.P. Zhang, S. Chen, Digital microfabrication of user-defined 3D microstructures in cell-laden hydrogels, Biotechnol. Bioeng. 110 (2013) 3038-3047. doi:10.1002/bit.24957.

[191] Y. Wu, Y.X. Chen, J. Yan, D. Quinn, P. Dong, S.W. Sawyer, P. Soman, Fabrication of conductive gelatin methacrylatete-polyaniline hydrogels, Acta Biomater. 33 (2016) 122-130. doi:10.1016/j.actbio.2016.01.036.

[192] S.W. Sawyer, P. Dong, S. Venn, A. Ramos, D. Quinn, J.A. Horton, P. Soman, Conductive gelatin methacrylate-poly(aniline) hydrogel for cell encapsulation, Biomed. Phys. Eng. Express. 4 (2018). doi:10.1088/2057-1976/aa91f9.

[193] A.K. Miri, D. Nieto, L. Iglesias, H. Goodarzi Hosseinabadi, S. Maharjan, G.U. Ruiz-Esparza, P. Khoshakhlagh, A. Manbachi, M.R. Dokmeci, S. Chen, S.R. Shin, Y.S. Zhang, A. Khademhosseini, Microfluidics-Enabled Multimaterial Maskless Stereolithographic Bioprinting, Adv. Mater. 30 (2018) 1-9. doi:10.1002/adma.201800242.

[194] Z. Wang, H. Kumar, Z. Tian, X. Jin, J.F. Holzman, F. Menard, K. Kim, Visible Light Photoinitiation of Cell-Adhesive Gelatin Methacryloyl Hydrogels for Stereolithography 3D Bioprinting, ACS Appl. Mater. Interfaces. 10 (2018) 26859-26869. doi:10.1021/acsami.8b06607.

[195] C.S. Bahney, T.J. Lujan, C.W. Hsu, M. Bottlang, J.L. West, B. Johnstone, Visible light photoinitiation of mesenchymal stem cell-laden bioresponsive hydrogels, Eur Cell Mater. 22 
(2016) 43-55. doi:10.1016/j.antiviral.2015.06.014.Chronic.

[196] J. Van Hoorick, H. Ottevaere, H. Thienpont, P. Dubruel, S. Van Vlierberghe, Polymer and Photonic Materials Towards Biomedical Breakthroughs, 2018. doi:10.1007/978-3-319-758015.

[197] X.-H. Qin, A. Ovsianikov, J. Stampfl, R. Liska, Additive manufacturing of photosensitive hydrogels for tissue engineering applications, BioNanoMaterials. 15 (2014) 49-70. doi:10.1515/bnm-2014-0008.

[198] A. Ovsianikov, V. Mironov, J. Stampf, R. Liska, Engineering 3D cell-culture matrices: multiphoton processing technologies for biological and tissue engineering applications., Expert Rev. Med. Devices. 9 (2012) 613-33. doi:10.1586/erd.12.48.

[199] W.R. Zipfel, R.M. Williams, W.W. Webb, Nonlinear magic: multiphoton microscopy in the biosciences, Nat. Biotechnol. 21 (2003) 1369-1377. doi:10.1038/nbt899.

[200] A. Ovsianikov, A. Deiwick, S. Van Vlierberghe, P. Dubruel, M. Lena, G. Dräger, B. Chichkov, Laser Fabrication of Three-Dimensional CAD Scaffolds from Photosensitive Gelatin for Applications in Tissue Engineering, Biomacromolecules. 12 (2011) 851-858.

[201] S. Engelhardt, E. Hoch, K. Borchers, W. Meyer, H. Krüger, G.E.M. Tovar, A. Gillner, Fabrication of 2D protein microstructures and 3D polymer-protein hybrid microstructures by two-photon polymerization., Biofabrication. 3 (2011) 025003. doi:10.1088/1758-5082/3/2/025003.

[202] S. Yoon, J.A. Park, H. Lee, W.H. Yoon, D.S. Hwang, Inkjet - Spray Hybrid Printing for 3D Freeform Fabrication of Multilayered Hydrogel Structures, 1800050 (2018) 1-10. doi:10.1002/adhm.201800050.

[203] V.H.M. Mouser, F.P.W. Melchels, J. Visser, W.J.A. Dhert, D. Gawlitta, J. Malda, Yield stress determines bioprintability of hydrogels based on gelatin-methacryloyl and gellan gum for cartilage bioprinting, Biofabrication. 8 (2016) 035003.

[204] P. Zhuang, W. Long Ng, J. An, C.K. Chua, L.P. Tan, Layer-by-layer ultraviolet assisted extrusionbased ( UAE ) bioprinting of hydrogel constructs with high aspect ratio for soft tissue 
engineering applications, PLoS One. (2019) 1-21.

[205] G. Cidonio, C.R. Alcala-orozco, K.S. Lim, M. Glinka, I. Mutreja, Y.-H. Kim, J.I. Dawson, T.B.F. Woodfield, R.O.C. Oreffo, Osteogenic and angiogenic tissue formation in high fidelity nanocomposite Laponite-gelatin bioinks, Biofabrication. 11 (2019) 035027.

[206] X. Zhou, W. Zhu, M. Nowicki, S. Miao, H. Cui, B. Holmes, R.I. Glazer, L.G. Zhang, 3D Bioprinting a Cell-Laden Bone Matrix for Breast Cancer Metastasis Study, ACS Appl. Mater. Interfaces. 8 (2016) 30017-30026. doi:10.1021/acsami.6b10673.

[207] K.S. Lim, R. Levato, P.F. Costa, M.D. Castilho, C.R. Alcala-Orozco, K.M.A. Van Dorenmalen, F.P.W. Melchels, D. Gawlitta, G.J. Hooper, J. Malda, T.B.F. Woodfield, Bio-resin for high resolution lithography-based biofabrication of complex cell-laden constructs, Biofabrication. 10 (2018). doi:10.1088/1758-5090/aac00c.

[208] K. Na, S. Shin, H. Lee, D. Shin, J. Baek, H. Kwak, M. Park, J. Shin, J. Hyun, Journal of Industrial and Engineering Chemistry Effect of solution viscosity on retardation of cell sedimentation in DLP 3D printing of gelatin methacrylate / silk fi broin bioink, J. Ind. Eng. Chem. 61 (2018) 340347. doi:10.1016/j.jiec.2017.12.032.

[209] S. Stier, L. Rebers, V. Schönhaar, E. Hoch, K. Borchers, Advanced formulation of methacryl- and acetyl-modi fi ed biomolecules to achieve independent control of swelling and stiffness in printable hydrogels, J. Mater. Sci. Mater. Med. (2019). doi:10.1007/s10856-019-6231-0.

[210] S. Sakai, K. Ueda, E. Gantumur, M. Taya, M. Nakamura, Drop-On-Drop Multimaterial 3D Bioprinting Realized by Peroxidase-Mediated Cross-Linking, Macromol. Rapid Commun. 39 (2018) 1-6. doi:10.1002/marc.201700534.

[211] S. Sakai, Y. Yamamoto, G. Enkhtuul, K. Ueda, K. Arai, M. Taya, M. Nakamura, Inkjetting Plus Peroxidase-Mediated Hydrogelation Produces Cell-Laden, Cell-Sized Particles with Suitable Characters for Individual Applications, Macromol. Biosci. 17 (2017) 1-6. doi:10.1002/mabi.201600416.

[212] K. Arai, Y. Tsukamoto, H. Yoshida, H. Sanae, T. Ahmad Mir, S. Sakai, T. Yoshida, M. Okabe, T. 
Nikaido, M. Taya, M. Nakamura, The development of cell-adhesive hydrogel for 3D printing, Int. J. Bioprinting. 2 (2017) 153-162. doi:10.18063/ijb.2016.02.002.

[213] F. Claeyssens, E. a Hasan, A. Gaidukeviciute, D.S. Achilleos, A. Ranella, C. Reinhardt, A. Ovsianikov, X. Shizhou, C. Fotakis, M. Vamvakaki, B.N. Chichkov, M. Farsari, Three-dimensional biodegradable structures fabricated by two-photon polymerization., Langmuir. 25 (2009) 3219-23.

[214] K. Obata, A. El-Tamer, L. Koch, U. Hinze, B.N. Chichkov, High-aspect 3D two-photon polymerization structuring with widened objective working range (WOW-2PP), Light Sci. Appl. 2 (2013) e116. doi:10.1038/Isa.2013.72.

[215] A.I. Ciuciu, P.J. Cywiński, Two-photon polymerization of hydrogels - versatile solutions to fabricate well-defined 3D structures, RSC Adv. 4 (2014) 45504-45516. doi:10.1039/C4RA06892K.

\section{Abbreviations}

$\begin{array}{ll}{ }^{1} \text { H-NMR } & \text { proton-Nuclear magnetic resonance spectroscopy } \\ \text { 2PP } & \text { two-photon polymerization } \\ \text { Ala } & \text { alanine } \\ \text { BMSC } & \text { Bone marrow stromal cells } \\ \text { CAD } & \text { Computer aided design } \\ \text { Cys } & \text { Cysteine } \\ \text { DAS } & \text { tetrapotassium 4,4'-(1,2-ethenediyl)bis(2-(3-sulfo- } \\ & \text { phenyl)diazenesulfonate) } \\ \text { DBA } & \text { diisobutylacrylamide } \\ \text { DLP } & \text { digital light projection } \\ \text { DMD } & \text { digital micromirror device } \\ \text { DS } & \text { degree of substitution } \\ \text { DSC } & \text { Differential Scanning Calorimetry } \\ \text { DTP } & 3,3^{\prime} \text {-dithiobis(propionic hydrazide) } \\ \text { DTT } & \text { Dithiotreitol } \\ \text { ECM } & \text { Extracellular matrix } \\ \text { FI } & \text { furfuryl isocyanate } \\ \text { FA } & \text { furfuryl amine } \\ \text { G' } & \text { Storage modulus } \\ \text { G" } & \text { Loss modulus } \\ \text { GAGs } & \text { glycosaminoglycans } \\ \text { gel-AA } & \text { gelatin-acrylamide } \\ \text { gel-AC } & \text { gelatin-anthracene } \\ \text { gel-AGE } & \text { gelatin allylglycidyl ether } \\ \text { gelatin-cys } & \text { gelatin-cystein } \\ \text { gelatin-Cys-2-MPD } & \text { gelatin modified with cystein and 2-mercaptopyrimidine-4,6 diol }\end{array}$




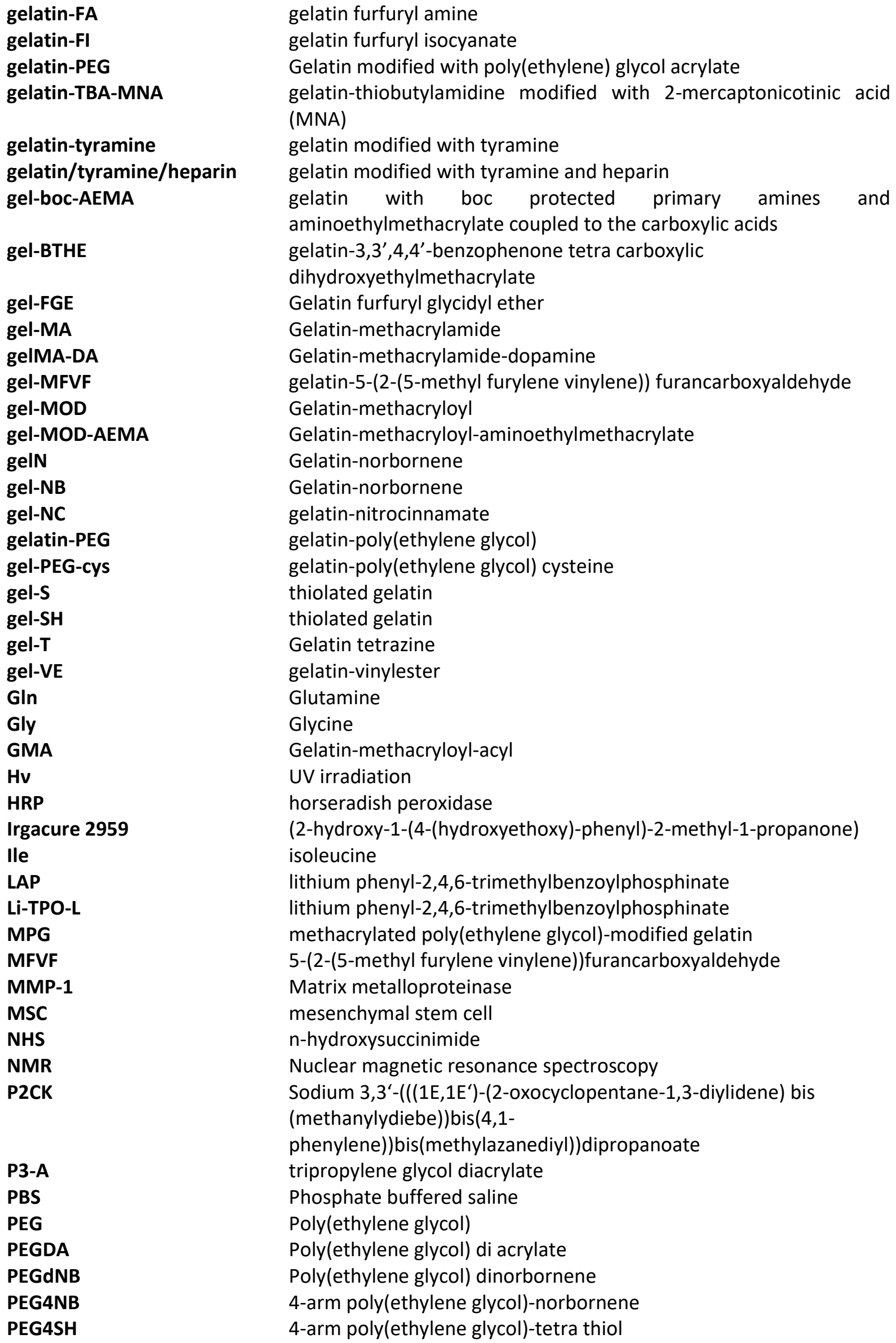


PEGTA

PI

PPA

Pro

PTA

PVA

PVA-MA

Ru/SPS

SLA

SPS

Td

$\mathrm{Tg}$

TTA

TNBSA

UDMA

UV

K-carrageenan-MA 4-arm poly(ethylene glycol) tetra acrylate

Photoinitiator

dipentaerythritol pentaacrylate

Proline

pentaerythritol triacrylate

Poly(vinyl alchohol)

Polyvinylalcohol-methacrylate

Photoinitiator based on a ruthenium complex (tris-bipyridylruthenium (II) hexahydrate) and sodium persulfate (SPS)

stereolithography

sodium persulfate

dissociation temperature

glass transition temperature

trimethylolpropane triacrylate

2,4,6-trinitrobenzene sulfonic acid

urethane-dimethacrylate

Ultraviolet

kappa-carrageenan-methacrylate 